\title{
EIGENVALUE PROBLEMS FOR SCHRÖDINGER-BOPP-PODOLSKY SYSTEMS
}

Lorena Soriano Hernandez

\section{THESE PRESENTED \\ TO THE}

INSTITUTE OF MATHEMATICS AND STATISTICS

OF THE

UNIVERSITY OF SÃO PAULO

TO

FULFILL THE REQUIREMENTS OF THE DEGREE

$\mathrm{OF}$

DOCTOR OF SCIENCE

GRADUATE PROGRAM: MATHEMATICS

ADVISOR: PhD. Gaetano Siciliano

During this project the author was supported by the agency CAPES and CNPq 


\section{Eigenvalue problems for Schrödinger-Bopp-Podolsky systems}

Esta versão da tese contém as correções e alterações sugeridas pela Comissão Julgadora durante a defesa da versão original do trabalho, realizada em 27/10/2021. Uma cópia da versão original está disponível no Instituto de Matemática e Estatística da Universidade de São Paulo.

Comissão Julgadora:

- Prof. Dr. Gaetano Siciliano (orientador) - IME-USP

- Prof. Dr. Paolo Piccione - IME-USP

- Prof. Dr. Marcos Tadeu de Oliveira Pimenta - FCT-UNESP

- Prof ${ }^{\mathrm{a}}$. Dr ${ }^{\mathrm{a}}$. Sandra Imaculada Moreira Neto - PPGMAT-UFMA

- Prof. Dr. Marcus Antônio Mendonça Marrocos - CMCC-UFABC 


\section{Acknowledgments}

I am immensely grateful to The University of São Paulo for contributing widely to my education as researcher.

I really appreciate the diligent disposition of Prof. Gaetano Siciliano for guiding me along this these. I really welcome his teachings. I have learned so much, thanks.

I would like to thank my family, María Blanca, María Esperanza and Juan Carlos, for their support and for cheering me at any time.

It was a pleasure to share intense study days, happy conquests and holidays with my colleagues. Thanks a lot to all of them.

I gratefully acknowledge financial support from CAPES.

Finally, I would like to thank Brazil for the opportunities and knowledge given to me not only in the field of mathematics. 


\section{Resumo}

\section{Soriano, H. L. PROBLEMAS DE AUTOVALORES PARA SISTEMAS}

SCHRÖDINGER-BOPP-PODOLSKY. 2021. Tese (Doutorado) - Instituto de Matemática e Estatística, Universidade de São Paulo, SP, 2021.

Esta tese trata um problema de autovalores para a equação de Schrödinger acoplada com o operador bi-harmônico. O problema modela uma partícula de massa movendo-se em um campo de uma força elétrica descrito pelo potencial $\phi$. O problema consiste em encontrar a existência de números reais $\omega$ e funções reais $u$ e $\phi$ que satisfazem o sistema

$$
\left\{\begin{aligned}
-\Delta u+\phi u & =\omega u \\
\Delta^{2} \phi-\Delta \phi & =u^{2}
\end{aligned} \quad \text { em } \Omega\right.
$$

sendo $\Omega$ um conjunto limitado e aberto de $\mathbb{R}^{N}, N \geq 3$, com as condições de fronteira e de normalização

$$
\left\{\begin{array}{l}
u=\Delta \phi=\phi=0 \quad \text { sobre } \quad \partial \Omega \\
\int_{\Omega} u^{2}=1 .
\end{array}\right.
$$

A função $\phi$ resulta pertencer ao espaço de Hilbert $H^{2}(\Omega) \cap H_{0}^{1}(\Omega)$. As soluções de $(1)$ se encontram analisando as equações de Euler-Lagrange do funcional

$$
F(u, \phi)=\frac{1}{2} \int_{\Omega}|\nabla u|^{2} d x+\frac{1}{2} \int_{\Omega} \phi u^{2} d x-\frac{1}{4} \int_{\Omega}|\Delta \phi|^{2} d x-\frac{1}{4} \int_{\Omega}|\nabla \phi|^{2} d x
$$

definido sobre a variedade $M=\left\{(u, \phi) \in H_{0}^{1}(\Omega) \times H^{2}(\Omega) \cap H_{0}^{1}(\Omega) ; \quad\|u\|_{L^{2}(\Omega)}=1\right\}$.

Nosso resultado principal é o seguinte enunciado. 
Teorema: Seja $\Omega$ um conjunto limitado e aberto de $\mathbb{R}^{N}, N \geq 3$. Então existe uma sequência $\left(\omega_{n}, u_{n}, \phi_{n}\right)$, tal que $\left(\omega_{n}\right) \subset \mathbb{R}, \omega_{n} \rightarrow \infty$ e $u_{n}, \phi_{n}$ são funções reais que resolvem (1) e (2). Além disso, $\left\|u_{n}\right\|_{H_{0}^{1}(\Omega)} \rightarrow \infty$.

Palavras-chave: Operador Bi-Lapaciano, Multiplicadores de Lagrange, Condição PS, Teoria do Gênero, Regularidade. 


\section{Abstract}

\section{Soriano, H. L. EIGENVALUE PROBLEMS FOR SCHRÖDINGER-}

BOPP-PODOLSKY SYSTEMS. 2021. Theses (PhD) - Institute of Mathematics and Statistics, University of São Paulo, SP, 2021.

This theses addresses an eigenvalues problem for the Schrödinger equation that involves the bi-harmonic operator. This problem models a single particle of mass moving under the influence of an electric force field described by a potential $\phi$. The problem is to find real numbers $\omega$ and real functions $u$ and $\phi$ satisfying the system

$$
\left\{\begin{array}{l}
-\Delta u+\phi u=\omega u \\
\Delta^{2} \phi-\Delta \phi=u^{2}
\end{array} \text { in } \Omega,\right.
$$

where $\Omega$ is a open bounded set of $\mathbb{R}^{N}, N \geq 3$, considering the boundary and normalizing conditions

$$
\left\{\begin{array}{l}
u=\Delta \phi=\phi=0 \quad \text { on } \quad \partial \Omega \\
\int_{\Omega} u^{2}=1 .
\end{array}\right.
$$

The function $\phi$ turns out to belong to the Hilbert space $H^{2}(\Omega) \cap H_{0}^{1}(\Omega)$. The solutions to (1) are found by working with the Euler-Lagrange equations of the functional

$$
F(u, \phi):=\frac{1}{2} \int_{\Omega}|\nabla u|^{2} d x+\frac{1}{2} \int_{\Omega} \phi u^{2} d x-\frac{1}{4} \int_{\Omega}|\Delta \phi|^{2} d x-\frac{1}{4} \int_{\Omega}|\nabla \phi|^{2} d x
$$

on the manifold $M=\left\{(u, \phi) \in H_{0}^{1}(\Omega) \times H^{2}(\Omega) \cap H_{0}^{1}(\Omega) ; \quad\|u\|_{L^{2}(\Omega)}=1\right\}$.

Our main result is the following theorem. 
Theorem: Let $\Omega$ be a open bounded set of $\mathbb{R}^{N}, N \geq 3$. There is a sequence $\left(\omega_{n}, u_{n}, \phi_{n}\right)$ such that $\left(\omega_{n}\right) \subset \mathbb{R}, \omega_{n} \rightarrow \infty$ and $u_{n}, \phi_{n}$ real functions solving (1) and (2). Furthermore, $\left\|u_{n}\right\|_{H_{0}^{1}(\Omega)} \rightarrow \infty$.

Keywords: Bi-Laplacian operator, Lagrange Multipliers, PS-Condition, Genus Theory, Regularity. 


\section{Contents}

1 Introduction $\quad 1$

2 Preliminaries $\quad 5$

2.1 Some Basic Results of Advanced Calculus . . . . . . . . . . . . . . . . . 5

2.2 Some Basic Results of Functional Analysis . . . . . . . . . . . . . . . . . . . 7

2.3 Spaces of Continuous Functions . . . . . . . . . . . . . . . . . 9

2.4 Sobolev Spaces . . . . . . . . . . . . . . . . . . . . . . 11

2.5 Differentiability of Weak Solutions . . . . . . . . . . . . . . . . . 17

2.6 Index Theory . . . . . . . . . . . . . . . . . . . . . . . . . . 18

2.6.1 Genus Theory . . . . . . . . . . . . . . . . 18

2.7 Regular Points and Submersions . . . . . . . . . . . . . . . . . . . . . . 19

2.8 Critical Point Theory . . . . . . . . . . . . . . . . . . . . . . . . 21

2.8.1 Critical Points and The Palais-Smale Condition . . . . . . . . . . . 21

2.8.2 Critical points under constraints and The Palais-Smale Condition . . 23

2.8 .3 Deformations . . . . . . . . . . . . . . . . . . 23

2.9 Trace Theory . . . . . . . . . . . . . . . . . . . . . 26

3 Some Physical Facts $\quad 29$

3.1 Variational Principle . . . . . . . . . . . . . . . . . . . . . . . . . . . 29

3.2 Eigenvalues and eigenfunctions of the Schrödinger equation . . . . . . . . . 30

3.3 Description of the solution of the Schrödinger equation . . . . . . . . . . 33

3.4 Non-linear Electrodynamics . . . . . . . . . . . . . . . . . . . . . . . 34

3.4.1 A system of Schr̈odinger-Bopp-Podolsky equations . . . . . . . . . . . 35

4 Solution of the SBP system $\quad 39$

4.1 Description of the solutions f . . . . . . . . . . . . . . . . . . . . 39

4.1.1 The space $\mathbb{H}_{\Delta^{2}}(\Omega)$. . . . . . . . . . . . . . . . . . . . . . . . . . . . 42

4.1 .2 Manifolds on Hilbert Spaces . . . . . . . . . . . . . . . . . . . . . 43

4.2 The variational principle for the SBP system . . . . . . . . . . . . . 44

4.3 The SBP system as a problem of eigenvalues . . . . . . . . . . . . . . . . 46

4.4 Elimination of variables . . . . . . . . . . . . . . . . . . . . 46 
4.5 Properties of the functional $J \ldots \ldots \ldots \ldots$

4.6 Main Result . . . . . . . . . . . . . . . . . . . . . . . . . . . 54

5 The SBP system with a parameter $\quad 57$

5.1 Solution of the SBP- $a$ system . . . . . . . . . . . . . . . . . . . 57

5.2 Regularity of solutions . . . . . . . . . . . . . . . . . . . . 59

5.3 The SBP-a system and a classical Schrödinger-Poisson system . . . . . . . 61

$\begin{array}{ll}\text { Bibliography } & 69\end{array}$ 


\section{Chapter 1}

\section{Introduction}

The outstanding problems of the systems of equations involving the bilaplacian operator have been extensively studied in recent years by various authors. There are some existence results for semilinear biharmonic non homogeneous on open and unbounded sets. C. O. Alves, João Marcos do Ò and O. H. Miyagaki in [COAM01] studied the class of semilinear biharmonic problems involving critical exponents:

$$
\Delta^{2} u+a(x) u=h(x)|u|^{q-1} u+k(x)|u|^{p-1} u \quad \text { in } \mathbb{R}^{N},
$$

where $N \geq 5,1<q<p \leq 2^{* *}-1=(N+4) /(N-4)$ and $a, h, k$ are bounded, non negative and continuous functions from $\mathbb{R}^{N}$ to $\mathbb{R}$. The authors overcame the lack of compactness using a version of the concentration compactness principle for the biharmonic operator, and then they showed the existence of a non trivial solution in $H^{2}\left(\mathbb{R}^{N}\right)$. Also, there are results about systems of equations coupled to the biharmonic operator.

P. d'Avenia and G. Siciliano in [dS19] studied a class of the Schrödinger-Bopp-Podolsky systems:

$$
\left\{\begin{array}{ll}
-\Delta u+\omega u+q^{2} \phi u & =|u|^{p-2} u \\
-\Delta \phi+a^{2} \Delta^{2} \phi & =4 \pi u^{2}
\end{array} \text { in } \mathbb{R}^{3},\right.
$$

with $a, \omega>0$. The authors proved the existence of non trivial solutions depending on the parameters $p$ and $q$, as well as the regularity of the solutions.

Additionaly, there are results about the convergence of solutions of systems of equations coupled with the biharmonic operator defined on a smooth closed 3-manifold $(M, g)$. Given 
the Bopp-Podolsky-Schrödinger-Proca system

$$
\left\{\begin{array}{ll}
\frac{\hbar^{2}}{2 m_{0}^{2}} \Delta_{g} u+\Phi(x, v, A) u & =u^{p-1} \\
a^{2} \Delta_{g}^{2} v+\Delta_{g} v+m_{a}^{2} v & =4 \pi q u^{2} \\
a^{2} \Delta_{g}^{2} A+\Delta_{g} A+m_{a}^{2} A & =\frac{4 \pi q \hbar}{m_{0}^{2}} \Psi(A, S) u^{2}
\end{array} \quad \text { in }(M, g),\right.
$$

with unknowns $(u, v, A)$, where $u$ and $v$ are functions, $u \geq 0$ in $M, A$ is a 1-form, $\Phi(x, v, A)=$ $\frac{\hbar^{2}}{2 m_{0}^{2}}|\Psi(A, S)|^{2}+\omega+q v=\nabla S-\frac{q}{\hbar} A$; also with $a, q, m_{0}, m_{1}$ positive numbers, $\omega$ a real number, $S$ is a given smooth real valued function on $M, \delta_{g}=-d i v_{g} \nabla$ is the Laplace-Beltrami operator acting on functions $u$ and $v, \Delta_{g}=\delta d+d \delta$ is the Hodge-de Rham Laplacian acting on the 1-form $A, \hbar$ is the Plank's constant and $p \in(2,6]$ ( $d$ is the differential, $\delta$ is the codifferential and 6 is the critical Sobolev exponent). E. Hebey proved in [Heb20] the strong convergence of the Bopp-Podolsky-Schrödinger-Proca system above to a Schrödinger-Poisson-Proca system when the Bopp-Podolsky parameter $a$ goes to zero with $p \in\left[\frac{22}{5}, 6\right]$.

Now, this work is framed in the study of a class of Schrödinger-Bopp-Podolsky systems. As a novelty, we apply topological methods to find solutions of equations that involve the bi-Laplacian operator as well as such solutions are defined in subsets of $\mathbb{R}^{N}$ where $N \geq 3$.

In the first chapter, we seek to mention the results that we will be used in the following chapters fixing the notations and the terminology that we will use throughout the work. We begin introducing some results on metric spaces, functional analysis and measure theory, then we present some results of index theory, critical point theory and trace theory. Here we seek global critical points as well as critical points in constricted sets, thus we begin presenting both the classical Palais-Smale condition and the Palais-Smale condition for critical points under constrictions. The last one is going to be used for development of this work. It should be mentioned that the deformation theorem contributes to obtain some of the main results.

In the second chapter, we offer a brief introduction of the concepts and principles of physics that describe the Schrödinger equation, the Bopp-Podolsky equation, and their boundary and normalizing conditions. We present the physical phenomena described by the equations of the Schrödinger-Bopp-Podolsky system, which also will help us to interpret the results obtained about the solutions of the system. 
In the third chapter, we will study the class of systems of partial differential equations of the form

$$
\left\{\begin{aligned}
-\Delta u+\phi u & =\omega u \\
\Delta^{2} \phi-\Delta \phi & =u^{2}
\end{aligned} \text { in } \Omega\right.
$$

with the boundary condition

$$
u=\Delta \phi=\phi=0 \quad \text { on } \quad \partial \Omega
$$

and normalizing condition

$$
\int_{\Omega} u^{2}=1
$$

where $\Omega$ is a bounded open subset of $\mathbb{R}^{N}$ and $N \geq 3$. In this case, a weak solution will be a positive real number $\omega$, a function $u$ that belongs to $H_{0}^{1}(\Omega)$ and a function $\phi$ that belongs to $H_{0}^{1}(\Omega) \cap H^{2}(\Omega)$.

One of the difficulties of this problem is to define the appropriate space of solution functions, so we dedicate the first section of the chapter to these spaces of solutions. The weak solutions of the Schrödinger-Bopp-Podolsky system above turn out to be precisely the critical points of the functional

$$
F(u, \phi)=\frac{1}{2} \int_{\Omega}|\nabla u|^{2} d x+\frac{1}{2} \int_{\Omega} \phi u^{2} d x-\frac{1}{4} \int_{\Omega}|\Delta \phi|^{2} d x-\frac{1}{4} \int_{\Omega}|\nabla \phi|^{2} d x
$$

defined on the manifold $M=\left\{(u, \phi) \in H_{0}^{1}(\Omega) \times \mathbb{H}_{\Delta^{2}} ;\|u\|_{2}=1\right\}$. Moreover, $F$ is a strongly indefinite functional, which means that $F$ is neither bounded from above nor from below. Therefore, the usual methods of the critical point theory can not be directly applied. To deal with this difficulty, we take advantage of the uniqueness of the solution of the equation $\Delta^{2} \phi-\Delta \phi=u^{2}$ to make the reduction of a variable of the functional $F$, thus obtaining a new (restricted) functional $J$ of the single variable $u$ defined on the ball $B=\left\{u \in H_{0}^{1}(\Omega) ;\|u\|_{2}=1\right\}$; following the technique used by Benci and Fortunato in [BF98].

Then, we will verify three facts about the functional $J: B \rightarrow \mathbb{R}$. First, $J$ satisfies the 
Palais-Smale condition for points under constraints, and we conclude that $J$ has critical points in $B$. Second, we use the invariance of genus to verify that every compact symmetric subset of $B$ has finite genus. Third, we apply the deformation lemma to guarantee that the level subset $J^{b}=\{u \in B, J(u) \leq b\}$, with $b$ a real number, also has finite genus. These facts imply the result of multiple solutions of the Schrödinger-Bopp-Podolsky system presented in Theorem 4.2 .

In the fourth chapter, based on the ideas of Chapter 4 we will obtain results about the multiple solutions for the Schrödinger-Bopp-Podolsky system

$$
\left\{\begin{array}{l}
-\Delta u+\phi u=\omega u \\
a^{2} \Delta^{2} \phi-\Delta \phi=u^{2}
\end{array} \text { in } \Omega\right.
$$

with the same boundary and normalizing conditions as before, where $a$ is a positive constant. Then, we analyze the regularity of the solutions of the Schrödinger-Bopp-Podolsky system obtaining strong solutions by the iterative application of the Sobolev immersions. Finally, we prove the strong convergence of the Schrödinger-Bopp-Podolsky system above to a Schrödinger-Poisson system when the Bopp-Podolsky parameter $a$ goes to zero. 


\section{Chapter 2}

\section{Preliminaries}

This chapter records necessary results to develop this work. We mention properties of Banach spaces. Then we introduce spaces of functions, namely those that are Hilbert, also their notations that will be kept along the work, as well as principal theorems about how those spaces are related. Finally, we review theories that will help us to achieve the solutions to our problem.

\subsection{Some Basic Results of Advanced Calculus}

This section is dedicated to recall some basic concepts and fundamental theorems of differential calculus on real Banach spaces. We present the derivative as a linear application. The bibliography devoted entirely to this subject are J. Dieudonné [Die69] and T. Schwartz [Shw69].

Definition 2.1 (Fréchet derivative). Let E, $F$ be real Banach spaces and $U$ an open subset of $E$. A continuous mapping $f$ of $U$ into $F$ is called Fréchet differentiable at $x_{0} \in U$ if there exists a bounded linear operator $L$ of $E$ into $F$ such that

$$
\lim _{h \rightarrow 0} \frac{f\left(x_{0}+h\right)-f\left(x_{0}\right)-L(h)}{h}=0 .
$$

The operator $L$ is called the Fréchet derivative of $f \in \mathcal{L}(E, F)$ at $x_{0}$, and it is written $f^{\prime}\left(x_{0}\right)$. The Fréchet derivative (when it exists) of a continuous mapping $f$ of $U$ into $F$, at a point $x_{0} \in U$, is thus an element of the Banach space $\mathcal{L}(E, F)$. In what follows, for $L \in \mathcal{L}(E, F)$ and $v \in E$, we will write $L\left(x_{0}\right)[v]$. 


\section{Example}

Let $E, F$ normed spaces. A continuous linear mapping $T$ of $E$ into $F$ is differentiable at every point $x$ and $T^{\prime}(x)=T$. Since $T$ is linear and continuous mapping

$$
T(x+h)=T(x)+T(h) .
$$

Observe that $L(h)[v]=0$ for all $v \in E$.

Definition 2.2. Let $f$ be a differentiable mapping of an open subset $U$ of a Banach space $E$ into a Banach space $F, f^{\prime}$ is the a mapping of $U$ into $\mathcal{L}(E, F)$. We say that $f$ is continuously differentiable in $U$ or of class $C^{1}$ if $f^{\prime}$ is continuous in $U$.

Theorem 2.1 (Chain Rule). Let E, F, G be three Banach spaces, $U$ an open neighborhood of $x_{0} \in E, f$ a continuous mapping of $U$ into $F, y_{0}=f\left(x_{0}\right), V$ an open neighborhood of $y_{0}$ in $F, g$ a continuous mapping of $B$ into $G$. Then if $f$ is differentiable at $x_{0}$ and $g$ differentiable at $y_{0}$, the mapping $h=g \circ f$ (which is defined and continuous in a neighborhood of $x_{0}$ ) is differentiable at $x_{0}$, and we have

$$
h^{\prime}\left(x_{0}\right)=g^{\prime}\left(y_{0}\right) \circ f^{\prime}\left(x_{0}\right)
$$

Definition 2.3 (Partial Derivatives). Suppose $E=E_{1} \times E_{2}$. For each point $(u, v) \in U$ we can consider the partial mappings $x \rightarrow f(x, v)$ and $y \rightarrow f(u, y)$ of open subsets of $E_{1}$ and $E_{2}$ respectively into $F$. We say that at $(u, v), f$ is differentiable with respect to the first (resp. second) variable if the partial mapping $x \rightarrow f(x, v)$ (resp. $y \rightarrow f(u, y)$ ) is differentiable at $u$ (resp. v), the derivative of that mapping, which is an element of $\mathcal{L}\left(E_{1}, F\right)$ (resp. $\mathcal{L}\left(E_{2}, F\right)$ ) is called the partial derivative of $f$ at $(u, v)$ with respect to the first (resp. second) variable, and written $f_{u}^{\prime}(u, v)$ (resp. $\left.f_{v}^{\prime}(u, v)\right)$.

Proposition 2.1. Let $E=E_{1} \times E_{2} \times \cdots \times E_{n}, F$ normed spaces provided with the norm $\|x\|_{E}=\sup \left\{\left\|x_{k}\right\|_{E_{k}} ; k=1,2, \ldots, n.\right\}$, where $x=\left(x_{1}, x_{2}, \ldots, x_{n}\right)$. Let $p: E \rightarrow F$ be a $n$-linear continuous mapping, then there exists $p^{\prime}(x)$ and

$$
p^{\prime}(x)[u]=\sum_{k=1}^{n} p\left(x_{1}, \ldots, x_{k-1}, u_{k}, x_{k+1}, \ldots, x_{n}\right) \text { for all } u=\left(u_{1}, u_{2}, \ldots, u_{n}\right) \in E
$$

Theorem 2.2. Let $f$ be a continuous mapping of an open subset $U$ of $E_{1} \times E_{2}$ into $F$. The map $f$ is continuously differentiable in $U$ if, and only if $f$ is differentiable at each point with respect to the first and the second variable, and the mappings $(x, y) \rightarrow f_{x}^{\prime}(x, y)$ and 
$(x, y) \rightarrow f_{y}^{\prime}(x, y)$ (of $U$ into $\mathcal{L}\left(E_{1}, F\right)$ and $\mathcal{L}\left(E_{2}, F\right)$ respectively) is continuous in $U$. Then, at each point $(x, y)$ of $U$, the (total derivative) derivative of $f$ is given by

$$
f^{\prime}(x, y)[u, v]=f_{x}^{\prime}(x, y)[u]+f_{y}^{\prime}(x, y)[v]
$$

Theorem 2.3 (Implicit Function). Let $E, F$ and $G$ be three Banach spaces, $f$ a continuously Fréchet differentiable mapping of an open subset $A$ of $E \times F$ into $G$. Let $\left(x_{0}, y_{0}\right)$ be a point of $A$ such that $f\left(x_{0}, y_{0}\right)=0$ and that the partial derivative $f_{y}^{\prime}\left(x_{0}, y_{0}\right)$ be a linear homeomorphism of $F$ onto $G$. Then there is an open neighborhood $U_{0}$ of $x_{0}$ in $E$ such that, for every open connected neighborhood $U$ of $x_{0}$, contained in $U_{0}$, there is a unique continuous mapping $u$ of $U$ into $F$ such that $u\left(x_{0}\right)=y_{0},(x, u(x)) \in A$ and $f(x, u(x))=0$ for any $x \in U$. Furthermore, $u$ is continuously differentiable in $U$, and its derivative is given by

$$
u^{\prime}(x)=-\left(f_{y}^{\prime}(x, u(x))\right)^{-1} \circ\left(f_{x}^{\prime}(x, u(x))\right)
$$

\subsection{Some Basic Results of Functional Analysis}

The results of this section belong to the classic theory of functional analysis. They can be found, for example, in E. Kreyszig [Kre78] and H. Brezis [Bre11].

Let $\Omega$ be an open set of $\mathbb{R}^{N}$, where $\partial \Omega$ denotes its boundary and $\bar{\Omega}$ is its closure.

Theorem 2.4 (Completion of metric spaces). For a metric space $X$ there exists a complete metric space $\hat{X}$ which has a subspace $W$ that is isometric with $X$ and is dense in $\hat{X}$. This space $\hat{X}$ is unique except for isometries, that is, if $\tilde{X}$ is any complete metric space having a dense subspace $\tilde{W}$ isometric with $X$, then $\tilde{X}$ and $\hat{X}$ are isometric.

Definition 2.4. Let $X$ be a normed space. Then the set of all continuous linear functionals on $X$ constitutes a normed space, $X^{\prime}$, with dual norm defined by

$$
\|f\|_{X^{\prime}}=\sup _{\substack{x \in X \\\|x\|=1}}|f(x)|
$$

Convergence of sequences of elements in a normed space is called strong convergence. It is reminded in order to distinguish it from weak convergence.

Definition 2.5. A sequence $\left(u_{k}\right)$ in a normed space $X$ is said to be strongly convergent (or convergent in the norm) if there is an $u \in X$ such that $\left\|u_{k}-u\right\|_{X} \rightarrow 0$, this is written $u_{k} \rightarrow u$. The element $u$ is called the strong limit of $\left(u_{k}\right)$, and we say that $\left(u_{k}\right)$ converges strongly to $u$. 
Weak convergence is defined in terms of bounded linear functionals on $X$ as follows.

Definition 2.6. A sequence $\left(u_{k}\right)$ in a normed space $X$ is said to be weakly convergent if there is an $u \in X$ such that for every $f \in X^{\prime}, f\left(u_{k}\right) \rightarrow f(u)$ as $k \rightarrow \infty$, this is written $u_{k} \rightarrow u$. The element $u$ is called the weak limit of $\left(u_{k}\right)$, and we say that $\left(u_{k}\right)$ converges weakly to $u$.

Theorem 2.5 (Banach-Alaoglu). Let $E$ be a reflexive Banach space. If $B \subset E$ is bounded, then $B$ is relatively compact in the weak topology of $E$.

Theorem 2.6 (Riesz's Representation). Let $H$ be a Hilbert space, and let $H^{\prime}$ be its topological dual. Then for every $f \in H^{\prime}$ there exists a unique $u_{f} \in H$ such that

$$
f[v]=\left(u_{f}, v\right)_{H} \text { for all } v \in H .
$$

Moreover, $\left\|u_{f}\right\|_{H}=\|f\|_{H^{\prime}}$. The linear application $R: H^{\prime} \rightarrow H$ that sends $f$ to $u_{f}$ is called the Riesz isomorphism.

Given $f \in E^{\prime}$ and $x \in E$ we shall often write $f[x]$ instead of $f(x)$.

Theorem 2.7. Lax-Milgram Assume that $a(u, v)$ is a continuous coercive bilinear form on $H$. Then, for every $\phi \in H^{\prime}$ there exists a unique element $u \in H$ such that

$$
a(u, v)=\phi[v] \quad \text { for all } v \in H .
$$

Moreover, if the form a is symmetric, then $u$ is characterized by the property

$$
u \in H \text { and } \frac{1}{2} a(u, u)-\phi[u]=\min _{v \in H}\left\{\frac{1}{2} a(v, v)-\phi[v]\right\}
$$

We finish this section with some important examples of Hilbert spaces of functions and the theorems about the convergence of function sequences.

The Lebesgue space of measurable functions $u: \Omega \rightarrow \mathbb{R}$ such that $\int_{\Omega}|u(x)|^{p} d x<+\infty$ is denoted by $L^{p}(\Omega)$, for each $p \in[1,+\infty)$. The norm that makes $L^{p}(\Omega)$ a Banach space is

$$
\|u\|_{p}:=\left(\int_{\Omega}|u(x)|^{p} d x\right)^{\frac{1}{p}} .
$$

When there is no confusion we shall often write $L^{p}$ instead of $L^{p}(\Omega)$.

Theorem 2.8 (Dominated convergence). Let $\Omega \subset \mathbb{R}^{N}$ be open and let $\left\{u_{k}\right\}_{k} \subset L^{1}(\Omega)$ be a sequence such that

1. $u_{k}(x) \rightarrow u(x)$ a.e. in $\Omega$ as $k \rightarrow \infty$; 
2. there exists $v \in L^{1}(\Omega)$ such that for all $k,\left|u_{k}(x)\right| \leq v(x)$ a.e. in $\Omega$.

Then $u \in L^{1}(\Omega)$ and $u_{k} \rightarrow u$ in the norm $L^{1}(\Omega)$, namely $\int_{\Omega}\left|u_{k}-u\right| d x \rightarrow 0$.

Theorem 2.9. Let $\Omega \subset \mathbb{R}^{N}$ be open and let $\left\{u_{k}\right\}_{k} \subset L^{p}(\Omega), p \in[1,+\infty)$, be a sequence such that $u_{k} \rightarrow u$ in $L^{p}(\Omega)$ as $k \rightarrow \infty$. Then there exist a subsequence $\left\{u_{k_{j}}\right\}_{j}$ and a function $v \in L^{p}(\Omega)$ such that

1. $u_{k_{j}}(x) \rightarrow u(x)$ a.e. in $\Omega$ as $j \rightarrow \infty$;

2. for all $j,\left|u_{k_{j}}(x)\right| \leq v(x)$ a.e. in $\Omega$.

Definition 2.7 (Locally Integrable Functions). A function u defined almost everywhere on $\Omega$ is said to be locally integrable on $\Omega$ provided

$$
\int_{K}|u(x)|^{p} d x<\infty, \text { for all compact subsets } K \text { of } \Omega \text {. }
$$

In this case we write $u \in L_{l o c}^{p}(\Omega)$, for each $p \in[1,+\infty)$.

Theorem 2.10. Let $\Omega \subset \mathbb{R}^{N}$ be an open set and let $u \in L_{\text {loc }}^{1}(\Omega)$ be such that

$$
\int u f=0, \quad \text { for all } f \in C_{0}^{\infty}(\Omega)
$$

then $u=0$ a. e. on $\Omega$.

Theorem 2.11 (Hölder's Inequality). Assume that $f \in L^{p}$ and $g \in L^{q}$ with $1 \leq p \leq \infty$, $\frac{1}{p}+\frac{1}{q}=1$. Then $f g \in L^{1}$ and

$$
\int|f g| \leq\|f\|_{p}\|g\|_{q}
$$

Theorem 2.12 (Extension of Hölder's Inequality). Assume that $f_{1}, \ldots, f_{k}$ are functions such that

$$
f_{i} \in L^{p_{i}}, 1 \leq i \leq k \text { with } \frac{1}{p}=\frac{1}{p_{1}}+\cdots+\frac{1}{p_{k}} \leq 1,
$$

then the product $f=f_{1} f_{2} \ldots f_{k}$ belongs to $L^{p}$ and

$$
\|f\|_{p} \leq\left\|f_{1}\right\|_{p_{1}}\left\|f_{2}\right\|_{p_{2}} \cdots\left\|f_{k}\right\|_{p_{k}} .
$$

\subsection{Spaces of Continuous Functions}

This section contains several definitions and results of spaces of continuous functions including standard notations; they provide the background required for what follows. For 
this, we consider R. A. Adams and J. Fournier [AF03] and D. Gilbarg and N. S. Trudinger [GT88] as references.

Let $\Omega \subset \mathbb{R}^{N}$ be an open set. We will deal with real valued functions on $\Omega$. We denote by $C(\Omega)$ the space of continuous functions on $\Omega$ and by $C^{m}(\Omega)$ the space of functions $m$ times continuously differentiable on $\Omega$. The space of the smooth functions is $C^{\infty}(\Omega)=\cap_{m} C^{m}(\Omega)$.

Observe that the functions in $C^{m}(\Omega)$ are not necessarily bounded. If $\phi \in C(\Omega)$ is bounded and uniformly continuous, then it possesses a unique, bounded, continuous extension to the closure of $\Omega$. Therefore, we define the vector space $C^{m}(\bar{\Omega})$ consisting of functions $\phi \in C^{m}(\Omega)$ for which $D^{\alpha} \phi$ is bounded and uniformly continuous for $0 \leq|\alpha| \leq m$.

For any non-negative integer $j$, let

$$
C_{B}^{j}(\Omega):=\left\{u \in C^{j}(\Omega) ; D^{\alpha} u \text { is bounded for }|\alpha| \leq j\right\} .
$$

Theorem 2.13. The space $C_{B}^{j}(\Omega)$ is larger than $C^{j}(\bar{\Omega})$.

Observe that the elements of $C_{B}^{j}(\Omega)$ are not necessarily uniformly continuous. However, we have

Theorem 2.14. $C_{B}^{j}(\Omega)$ is a Banach space under the norm

$$
\|u\|_{C_{B}^{j}(\Omega)}:=\max _{0 \leq|\alpha| \leq j} \sup _{x \in \Omega}\left|D^{\alpha} u(x)\right|
$$

Definition 2.8. A real valued function $f$ satisfies the Hölder condition when there are non-negative constants $K$ and $\lambda$, such that

$$
|f(x)-f(y)| \leq K\|x-y\|^{\lambda}
$$

for all $x$ and $y$ in the domain of $f$.

Definition 2.9. If $0<\lambda \leq 1$, the vector space $C^{j, \lambda}(\bar{\Omega})$ consists the space of the functions $\phi \in C^{j}(\bar{\Omega})$ for which $D^{\alpha} \phi$ is bounded and uniformly continuous on $\Omega$ for $0 \leq|\alpha| \leq j$, where each function $D^{\alpha} \phi$ satisfies on $\Omega$ the Hölder condition with exponent $\lambda$.

Theorem 2.15. The space $C^{j, \lambda}(\bar{\Omega})$ is a Banach space with the norm given by

$$
\|\phi\|_{C^{j, \lambda}(\bar{\Omega})}:=\max _{0 \leq|\alpha| \leq j} \sup _{x \in \Omega}\left|D^{\alpha} u(x)\right|+\max _{0 \leq|\alpha| \leq j} \sup _{\substack{x, y \in \Omega \\ x \neq y}} \frac{\left|D^{\alpha} \phi(x)-D^{\alpha} \phi(y)\right|}{|x-y|^{\lambda}} .
$$

Definition 2.10. We denotes by $C_{0}(\Omega)$ the space of continuous functions on $\Omega$ with compact support, $i$. e., the functions that vanish outside some compact set $K \subset \Omega$.

Finally we denote by $C_{0}^{m}(\Omega):=C^{m}(\Omega) \cap C_{0}(\Omega)$ the space of $m$ times functions differentiate with compact support and by $C_{0}^{\infty}(\Omega):=C^{\infty}(\Omega) \cap C_{0}(\Omega)$ the space of smooth functions with compact support. 


\subsection{Sobolev Spaces}

This section displays the appropriated spaces of functions to set the solutions of our nonlinear problem. Also, we mention some properties of Sobolev spaces. For this part, we consider the authors R. A. Adams and J. Fournier [AF03], H. Brezis [Bre11] and S. Kesavan [Kes89].

In this section, $\Omega$ stands for an open set in $\mathbb{R}^{N}$ and $\partial \Omega$ for its boundary.

Definition 2.11. Let $m>0$ be an integer and let $1 \leq p \leq \infty$. The corresponding Sobolev space is given by

$$
W^{m, p}(\Omega):=\left\{u \in L^{p}(\Omega) ; D^{\alpha} u \in L^{p}(\Omega) \text { for all }|\alpha| \leq m\right\} .
$$

In other words, $W^{m, p}(\Omega)$ is the collection of all functions in $L^{p}(\Omega)$ such that all distribution derivatives up to order $m$ are also in $L^{p}(\Omega)$. In the case $m=1$, we have the following result.

Theorem 2.16. The Sobolev space $W^{1, p}(\Omega)$ is

$$
W^{1, p}(\Omega)=\left\{u \in L^{p}(\Omega) ; \quad \begin{array}{c}
\text { there exist } g_{1}, \ldots, g_{N} \in L^{p}(\Omega) \text { such that } \\
\int_{\Omega} u \frac{\partial \varphi}{\partial x_{i}}=-\int_{\Omega} g_{i} \varphi \forall \in \in C_{0}^{\infty}(\Omega), \forall i=1,2, \ldots, N
\end{array}\right\} .
$$

In particular, when $m=1$ and $p=2$, the Sobolev space acquire a single notation

$$
H^{1}(\Omega):=W^{1,2}(\Omega) .
$$

When there is no confusion we shall often write $H^{1}$ instead of $H^{1}(\Omega)$. From Definition 2.16, we obtain integrating by parts

$$
\int_{\Omega} u \frac{\partial \varphi}{\partial x_{i}}=-\int_{\Omega} \frac{\partial u}{\partial x_{i}} \varphi
$$

then

$$
\int_{\Omega} g_{i} \varphi=\int_{\Omega} \frac{\partial u}{\partial x_{i}} \varphi
$$

and by Theorem 2.10

$$
g_{i}=\frac{\partial u}{\partial x_{i}} \text { a. e. in } \Omega, \quad \text { for all } i=1, \ldots, n \text {. }
$$

Therefore, the gradient of $u$

$$
\nabla u:=\operatorname{grad} u=\left(\frac{\partial u}{\partial x_{1}}, \frac{\partial u}{\partial x_{2}}, \ldots, \frac{\partial u}{\partial x_{N}}\right)
$$

Therefore, the gradient is used to define the inner product in $H^{1}(\Omega)$. 
Theorem 2.17. The space

$$
H^{1}(\Omega)=\left\{u \in L^{2}(\Omega) ; \nabla u \in L^{2}(\Omega)\right\}
$$

is a Hilbert space with the scalar product

$$
(u, v)_{H^{1}}:=\int_{\Omega}(\nabla u \nabla v+u v) d x=\sum_{i=1}^{N} \int_{\Omega} \frac{\partial u}{\partial x_{i}} \frac{\partial v}{\partial x_{i}}+\int_{\Omega} u v
$$

The corresponding norm in $H^{1}(\Omega)$ is

$$
\|u\|_{H^{1}}:=\left(\int_{\Omega}\left(|\nabla u|^{2}+|u|^{2}\right) d x\right)^{\frac{1}{2}} .
$$

Definition 2.12. Let $1 \leq p<\infty ; W_{0}^{1, p}(\Omega)$ is defined as the closure of $C_{0}^{\infty}(\Omega)$ with the norm in $W^{1, p}(\Omega)$. We denote

$$
H_{0}^{1}(\Omega):=W_{0}^{1,2}(\Omega) .
$$

Theorem 2.18. The space $W_{0}^{1, p}(\Omega)$ equipped with the norm in $W^{1, p}(\Omega)$ is a separable Banach space. It is reflexive if $1<p<\infty$. The space $H_{0}^{1}(\Omega)$ equipped with the scalar product of $H^{1}(\Omega)$ is a Hilbert space.

Let us consider another particular Sobolev space

$$
H^{2}(\Omega):=W^{2,2}(\Omega),
$$

it is the collection of all functions in $L^{2}(\Omega)$ such that all distribution derivatives up to order 2 are also in $L^{2}(\Omega)$.

Theorem 2.19. The space

$$
H^{2}(\Omega)=\left\{u \in L^{2}(\Omega) ; \quad D^{|\alpha|} u \in L^{2}(\Omega),|\alpha| \leq 2\right\},
$$

is a Hilbert space with the scalar product

$$
(u, v)_{H^{2}}:=\sum_{|\alpha| \leq 2} \int_{\Omega} D^{\alpha} u D^{\alpha} v .
$$

The corresponding norm in $H^{2}(\Omega)$ is

$$
\|u\|_{H^{2}}:=\left(\sum_{|\alpha| \leq 2} \int_{\Omega}\left|D^{\alpha} u\right|^{2}\right)^{\frac{1}{2}}
$$


Definition 2.13 (Embeddings). A Banach space $E$ is embedded continuously in a Banach space $F$ if

1. $E \subseteq F$;

2. the canonical injection $j: E \rightarrow F$ is a continuous operator. This means that there exists a constant $C>0$ such that

$$
\|u\|_{F} \leq C\|u\|_{E}, \text { for all } u \in E \text {. }
$$

We denote this by $E \hookrightarrow F$.

Definition 2.14. A Banach space $E$ is compactly embedded in a Banach space $F$ if $E$ is embedded continuously in $F$ and the canonical injection is a compact operator.

Theorem 2.20. Let $\Omega$ be an open bounded subset of $\mathbb{R}^{N}$, with $N \geq 3$. Then

$$
H_{0}^{1}(\Omega) \hookrightarrow L^{q}(\Omega) \text { for every } q \in\left[1, \frac{2 N}{N-2}\right]
$$

The embedding is compact if and only if $q \in\left[1, \frac{2 N}{N-2}\right)$.

We point out that for each $q \in\left[1, \frac{2 N}{N-2}\right]$, the continuity of the above embedding is explicitly expressed by inequalities of the form

$$
\|u\|_{q} \leq C\|u\|_{H^{1}}, \text { for all } u \in H_{0}^{1}(\Omega)
$$

where $C$ does not depend on $u$.

The number $\frac{2 N}{N-2}$ is denoted by $2^{*}$ and is called the critical Sobolev exponent for the embedding $H_{0}^{1} \hookrightarrow L^{q}$. The term "critical " refers to the fact that the embedding of the preceding theorem fails for $q>2^{*}$.

The set $\Omega \subset \mathbb{R}^{N}$ satisfies the cone condition if there exists a finite cone $C$ such that each $x \in \Omega$ is the vertex of a finite cone $C_{x}$ contained in $\Omega$ and congruent to $C$.

The set $\Omega \subset \mathbb{R}^{N}$ satisfies the strong local Lipschitz condition if there exist positive numbers $\delta$ and $M$, a locally finite open cover $U_{j}$ of the boundary of $\Omega$, and for each $j$ a real-valued function $f_{j}$ of $N-1$ variables, such that the following conditions hold:

1. Every collection of $R+1$ sets $U_{1}, U_{2}, \ldots U_{R+1}$ has empty intersection. 
2. For every pair of points $x, y \in \Omega_{\delta}:=\{x \in \Omega$; $\operatorname{dist}(x, \partial \Omega)<\delta\}$ satisfying $|x-y|<\delta$, there exists $j$ such that

$$
x, y \in V_{j}:=\left\{x \in U_{j} ; \operatorname{dist}\left(x, \partial U_{j}\right)>\delta\right\} .
$$

3. Each function $f_{j}$ satisfies a Lipschitz condition with constant $M$, that is,

$$
\left|f_{j}(\xi)-f_{j}(\rho)\right| \leq M|\xi-\rho|, \text { for all } \xi, \rho \in \mathbb{R}^{N-1}
$$

4. For some Cartesian coordinate system $\left(\varphi_{j, 1}, \ldots, \varphi_{j, N}\right)$ in $U_{j}$,

$$
\Omega \cap U_{j} \subset\left\{\left(\zeta_{j, 1}, \ldots, \zeta_{j, N}\right) ; \zeta_{j, N}<f_{j}\left(\zeta_{j, 1}, \ldots, \zeta_{j, N-1}\right)\right\}
$$

If $\Omega$ is bounded, the rather complicated set of conditions above reduce to the simple condition that $\Omega$ should have a locally Lipschitz boundary, that is, that each point $x$ on the boundary of $\Omega$ should have a neighborhood $U_{x}$ whose intersection with $\partial \Omega$ should be the graph of a Lipschitz continuous function.

The next theorem establishes some important properties of Sobolev spaces. The functions of those spaces are defined on an arbitrary domain $\Omega$, that is, a non-empty open set in $\mathbb{R}^{N}$.

Theorem 2.21 (The Sobolev embedding theorem). Let $\Omega$ be a domain in $\mathbb{R}^{N}$ and let $\Omega^{k}$ the $k$-dimensional domain obtained by intersecting $\Omega$ with a $k$-dimensional plane in $\mathbb{R}^{N}$, $1 \leq k \leq N$, thus $\Omega^{N}=\Omega$. Let $j$ and $m$ be non-negative integers and let $p$ satisfy $1 \leq p<\infty$.

\section{PART I}

If $\Omega$ has the cone property, then there exist the following embeddings:

CASE A: Suppose $m p=N$ and $N-m p<k \leq N$. Then

$$
\text { if } p \leq q \leq \frac{k p}{N-m p}, W^{j+m, p}(\Omega) \hookrightarrow W^{j, q}\left(\Omega^{k}\right),
$$

and in particular,

$$
\text { if } p \leq q \leq \frac{N p}{N-m p}, W^{j+m, p}(\Omega) \hookrightarrow W^{j, q}(\Omega)
$$

or

$$
\text { if } p \leq q<\frac{N p}{N-m p}, W^{m, p}(\Omega) \hookrightarrow L^{q}(\Omega) .
$$

Moreover, if $p=1$ and $m<N$, embedding (2.1) exists for $k=N-m$.

CASE B: Suppose $m p=N$. Then for each $k, 1 \leq k \leq N$,

$$
\text { if } p \leq q<\infty, W^{j+m, p}(\Omega) \hookrightarrow W^{j, q}\left(\Omega^{k}\right)
$$


so that in particular,

$$
\text { if } p \leq q<\infty, W^{j+m, p}(\Omega) \hookrightarrow W^{j, q}(\Omega)
$$

Moreover, if $p=1$ so that $m=N$, embeddings (2.4) and (2.5) exist with $q=\infty$ as well; in fact,

$$
W^{j+N, 1}(\Omega) \hookrightarrow C_{B}^{j}(\Omega)
$$

CASE C: Suppose $m p>N$. Then

$$
W^{j+m, p}(\Omega) \hookrightarrow C_{B}^{j}(\Omega)
$$

The embedding constants for the embeddings above depend only on $N, m, p, q, j, k$, and the dimensions of the cone $C$ in the cone condition.

\section{PART II}

If $\Omega$ has the strong local Lipschitz property, then CASE C of PART I can be written as follows:

CASE C.1: If $m p>N>(m-1) p$, then

$$
\text { if } 0<\lambda \leq m-\left(\frac{N}{p}\right), W^{j+m, p}(\Omega) \hookrightarrow C^{j, \lambda}(\bar{\Omega})
$$

CASE C.2: If $N=(m-1) p$, then

$$
\text { if } 0<\lambda<1, W^{j+m, p}(\Omega) \hookrightarrow C^{j, \lambda}(\bar{\Omega}) \text {. }
$$

Also, if $N=m-1$ and $p=1$, then (2.9) holds for $\lambda=1$ as well.

\section{PART III}

All of the embeddings in $\boldsymbol{P A R T} \boldsymbol{I}$ and $\boldsymbol{P A R T} \boldsymbol{I I}$ are valid for arbitrary domains $\Omega$ if the $W$-space undergoing the embedding is replaced with the corresponding $W_{0}$-space.

Theorem 2.22 (Poincaré inequality). Let $\Omega \subset \mathbb{R}^{N}$ be open and bounded. Then there exists a constant $C>0$, depending only on $\Omega$, such that

$$
\int_{\Omega} u^{2} d x \leq C \int_{\Omega}|\nabla u|^{2} d x, \text { for all } u \in H_{0}^{1}(\Omega) .
$$

Theorem 2.23 (Classical Green's formula). Let $\Omega \subset \mathbb{R}^{N}$ be open, bounded and smooth. Let $u \in C^{2}(\bar{\Omega})$ and $v \in C^{1}(\bar{\Omega})$. Then

$$
\int_{\Omega}(\Delta u) v d x=\int_{\partial \Omega} \frac{\partial u}{\partial \nu} v d \sigma-\int_{\Omega} \nabla u \cdot \nabla v d x
$$


where $\nu=\nu(x)$ is the outward normal to $\partial \Omega$ at $x, \frac{\partial u}{\partial \nu}(x)=\nabla u(x) \cdot \nu(x)$ and $\sigma$ is the surface measure on $\partial \Omega$.

In order to define the dual space of $H_{0}^{1}(\Omega)$ we recall two definitions about operators. Let $A: D(A) \subset E \rightarrow F$ be a linear operator, it is densely defined if the domain of $A, D(A)$, is dense in $E$. Furthermore, if $A$ is an unbounded linear operator that is densely defined, we define an unbounded operator $A^{\prime}: D\left(A^{\prime}\right) \subset F^{\prime} \rightarrow E^{\prime}$ as follows. We define its domain

$$
D\left(A^{\prime}\right):=\left\{v \in F^{\prime} ; \text { there exists } c \geq 0 \text { such that }|(v, A u)| \leq c\|u\|_{E} \text {, for all } u \in D(A)\right\} .
$$

The domain of $A^{\prime}$ turns out to be a linear subspace of $F^{\prime}$. Now, we define $A^{\prime} v$. Given $v \in D\left(A^{\prime}\right)$, consider the map $g: D(A) \rightarrow \mathbb{R}$ defined by

$$
g(u)=(v, A u), \text { for all } u \in D(A) .
$$

We have

$$
|g(u)| \leq c\|u\|_{E}, \text { for all } u \in D(A)
$$

By Hahn-Banach Theorem, there exists a linear functional $f: E \rightarrow \mathbb{R}$ that extends $g$ and such that

$$
|f(u)| \leq c\|u\|_{E} \text {, for all } u \in E .
$$

It follows that $f \in E^{\prime}$. Note that the extension of $g$ is unique since $D(A)$ is dense in $E$. Then, we set

$$
A^{\prime} v=f .
$$

The unbounded linear operator $A^{\prime}: D\left(A^{\prime}\right) \subset F^{\prime} \rightarrow E^{\prime}$ is called the adjoint of $A$.

The fundamental relation between $A$ and $A^{\prime}$ is given by

$$
(v, A u)_{F^{\prime}, F}=\left(A^{\prime} v, u\right)_{E^{\prime}, E} \text {, for all } u \in D(A) \text { and for all } v \in D\left(A^{\prime}\right)
$$

Definition 2.15. Let $1 \leq p<\infty$. Let $p^{\prime}$ satisfying $\frac{1}{p}+\frac{1}{p^{\prime}}=1$. The dual space of $W_{0}^{m, p}(\Omega)$, where $m \geq 1$ is an integer, is denoted by $W_{0}^{-m, p^{\prime}}(\Omega)$. If $m=1$ and $p=2, H^{-1}(\Omega)$ is the dual space of $H_{0}^{1}(\Omega)$.

Theorem 2.24. The dual space $H^{-1}(\Omega)$ is a Hilbert space. In the case of $L^{2}(\Omega)$ it is identified with its own dual. The following inclusions are dense and continuous

$$
H_{0}^{1}(\Omega) \hookrightarrow L^{2}(\Omega) \stackrel{\cong}{\leftrightarrows} L^{2^{\prime}}(\Omega) \hookrightarrow H^{-1}
$$




\subsection{Differentiability of Weak Solutions}

This section is devoted to regularity of the weak solutions of elliptic equations. We present differentiability results about of the weak solutions of high order equations. Those equations are given by linear elliptic operators having principal part in divergence form under assumptions of relatively weak smoothness on the coefficients. The next results were taken from D. Gilbarg and N. S. Trudinger [GT88].

We consider operators $L$ of the form

$$
L u=D_{i}\left(a^{i j}(x) D_{j} u+b^{i}(x) u\right)+c^{i}(x) D_{i} u+d(x) u, \quad i, j=1, \ldots N
$$

whose coefficients $a^{i j}, b^{i}, c^{i}, d$ are assumed to be measurable functions on a domain $\Omega \subset \mathbb{R}^{N}$. Let $f^{i}, i=1, \ldots, N$ be locally integrable functions in $\Omega$. Then, a weakly differentiable function $u$ will be called a weak solution of the equation

$$
L u=D_{i} f^{i} \text { in } \Omega
$$

if

$$
\langle L u, v\rangle=\int_{\Omega} f^{i} D_{i} v, \text { for all } v \in C_{0}^{1}(\Omega) .
$$

Also, we assume that $L$ is strictly elliptic in $\Omega$, that is, there exists a positive number $\lambda$ such that

$$
a^{i j}(x) \xi_{i} \xi_{j} \geq \lambda(x)|\xi|^{2}, \text { for all } x \in \Omega \text { and for all } \xi=\left(\xi_{1}, \ldots, \xi_{n}\right) \in \mathbb{R}^{N} \backslash\{0\}
$$

We also assume that $L$ has bounded coefficients, i.e. there exist constants $\Lambda$ and $\nu \geq 0$ such that

$$
\sum_{i=1}^{N} \sum_{j=1}^{N}\left|a^{i j}(x)\right|^{2} \leq \Lambda^{2} \quad \text { and } \quad \lambda^{-2} \sum_{i=1}^{N}\left(\left|b^{i}(x)\right|^{2}+\left|c^{i}(x)\right|^{2}\right)+\lambda^{-1}|d(x)| \leq \nu^{2}, \text { for all } x \in \Omega
$$

Theorem 2.25. Let $u \in H^{1}(\Omega)$ be a weak solution of the equation $L u=f$ in $\Omega$, where $L$ is strictly elliptic in $\Omega$, the coefficients $a^{i, j}, b^{i} \in C^{m, 1}(\bar{\Omega})$, the coefficients $c^{i}, d \in C^{m-1,1}(\bar{\Omega})$ and the function $f \in W^{m, 2}(\Omega), m \geq 1$. Then for any sub-domain $\Omega^{\prime} \subset \Omega$ with compact closure in $\Omega$, we have $u \in W^{m+2,2}\left(\Omega^{\prime}\right)$ and

$$
\|u\|_{W^{m+2,2}\left(\Omega^{\prime}\right)} \leq C\left(\|u\|_{H^{1}(\Omega)}+\|f\|_{W^{m, 2}(\Omega)}\right) \text { for } C=C(N, \lambda, m, K),
$$

where $K=\max \left\{\left\|a^{i, j}\right\|_{C^{m, i}(\bar{\Omega})},\left\|b^{i}\right\|_{C^{m, i}(\bar{\Omega})},\left\|c^{i}\right\|_{C^{m-1,1}(\bar{\Omega})},\|d\|_{C^{m-1,1}(\bar{\Omega})}\right\}$.

Theorem 2.26. Let $f \in L^{p}, 1<p<\infty$, and let $w$ be the Newtonian potential of $f$. Then 
$w \in W^{2, p}(\Omega), \Delta w=f$ a. e. and

$$
\left\|D^{2} w\right\|_{p} \leq C\|f\|_{p}
$$

where $C$ depends only on $N$ and $p$. Furthermore, when $p=2$ we have

$$
\int_{\mathbb{R}^{N}}\left|D^{2} w\right|=\int_{\Omega} f^{2}
$$

\subsection{Index Theory}

Index theory comprises the study of invariants in topological spaces. These invariants suitably characterize the spaces in order to apply some methods of the Critical Point Theory. Among these invariants are known the topological Degree, Ljusternik-Schnirelman Category and the Krasnoselskii Genus. For the particular interest of this work, in this section we present the principal results about Genus Theory. Details of General Index Theory can be seen in P. H. Rabinowitz [Rab74], M. Struwe [Str90] and Coffman [Cof69].

\subsubsection{Genus Theory}

Let $E$ be a real Banach space and the class

$$
\mathcal{A}:=\{A \subset E \backslash\{0\} ; A \text { closed in } E \text { and }-A=A\} .
$$

Let $A \in \mathcal{A}$ be non-empty. If there exist odd maps in $C\left(A ; \mathbb{R}^{N} \backslash\{0\}\right)$, we can define

$$
G:=\left\{N \geq 1 ; \text { there exists } h \in C\left(A ; \mathbb{R}^{N} \backslash\{0\}\right) \text { such that } h(-u)=-h(u)\right\} .
$$

The genus of $A$ is defined by

$$
\gamma(A):=\left\{\begin{array}{cl}
\min G, & \text { if } G \neq \emptyset \\
\infty, & \text { if } G=\emptyset
\end{array} .\right.
$$

If $A=\emptyset$, we write $\gamma(A)=0$. In the case $0 \in A$ we say that $\gamma(A)=\infty$.

We describe some properties of the genus in the following proposition.

Proposition 2.2. Let $A, B \in \mathcal{A}$. Then the following hold:

1. $\gamma(A) \geq 0$, and $\gamma(A)=0$ if and only if $A=\emptyset$.

2. $A \subset B, \gamma(A) \leq \gamma(B)$.

3. Let $h \in C(E, E)$ be an odd map, then $\gamma(A) \leq \gamma(\overline{h(A)})$. 
4. $\gamma(A \cup B) \leq \gamma(A)+\gamma(B)$.

5. If $A \in \mathcal{A}$ is compact, then $\gamma(A)<\infty$ and there exists $\delta>0$ such that $\overline{N_{\delta}(A)} \in \mathcal{A}$ and $\gamma\left(\overline{N_{\delta}(A)}\right)=\gamma(A)$, where $N_{\delta}(A):=\{x \in E ; \quad \operatorname{dist}(x, A) \leq \delta\}$.

6. If there is an odd homeomorphism $h \in C(A, B)$, then $\gamma(A)=\gamma(B)$.

7. If $\gamma(B)<\infty, \gamma(\overline{A \backslash B}) \geq \gamma(A)-\gamma(B)$.

8. If $\gamma(A)>k, V$ is a $k$-dimensional subspace of $E$, and $V^{\perp}$ is an algebraically and topologically complementary subspace, then $A \cap V^{\perp} \neq \emptyset$.

The notion of genus generalizes the notion of dimension of a linear space as the following result shows.

Theorem 2.27. Let $\Omega$ be bounded, open and symmetric subset in $\mathbb{R}^{N}$ with $0 \in \Omega$ and $A \in \mathcal{A}$. If there exists an odd homeomorphism $h: A \rightarrow \partial \Omega$, then $\gamma(A)=N$.

Corollary 2.27.1. Let $A \in \mathcal{A}$. If there exists an odd homeomorphism from $A$ to the sphere of $\mathbb{R}^{N}, \mathbb{S}^{N-1}$, then $\gamma(A)=N$.

\subsection{Regular Points and Submersions}

Although, we are interested in the critical points of a functional, its regular points are not less important thanks to the useful property of regular-level sets. In this section we characterize such regular-level sets as manifolds of infinite dimension. An entire description of this theory is done in E. Zeidler [Zei84] and [Zei88], T. Schwartz [Shw69], P. Blanchard and E. Brüning [BB92] and L. Ljusternik and L. Schnirelman [LS34].

Definition 2.16. Let $E$ be a Banach space, $U$ an open set of $E, J \in C^{1}(U, \mathbb{R})$ and $u \in U$. If $J^{\prime}(u)$ is a surjective function, then $u \in U$ is a regular point of $J$. A real number $c$ is a regular value of $J$ if every point of the level set $J^{-1}(c)$ is a regular point. In particular, if $J^{-1}(c)=\emptyset$, then $c$ is a regular value. Finally, a level set $J^{-1}(c)$ is called a regular level set if $c$ is a regular value of $J$.

Definition 2.17. If $E, F$ are real Banach spaces, then a map $G: D(G) \subseteq E \rightarrow F$ is called a submersion at $u_{0} \in D(G)$ if and only if the following hold:

1. G is continuously Fréchet differentiable in a neighborhood of $u_{0}$,

2. $G^{\prime}\left(u_{0}\right): E \rightarrow F$ is surjective, 
3. The null space $\operatorname{Ker}\left(G^{\prime}\left(u_{0}\right)\right)$ splits $E$

$$
E=\operatorname{Ker}\left(G^{\prime}\left(u_{0}\right)\right) \oplus(I-P)(E),
$$

where $P$ is a continuous projection operator of $E$ on $\operatorname{Ker}\left(G^{\prime}\left(u_{0}\right)\right)$.

In differential geometry, we know that every regular level set of a smooth map between smooth manifolds is a properly embedded submanifold. The next result is a generalization for manifolds over real Banach spaces (not necessarily of finite dimension).

Definition 2.18. Let $V$ be a linear topological space and $M$ a topological space. A smooth $V$-structure on $M$ is a pair $(\mathfrak{U}, \mathfrak{J})$, where

1. $\mathfrak{U}=\left\{U_{\alpha}\right\}$ is an open covering of $M$.

2. $\mathfrak{J}=\left\{\theta_{\alpha}\right\}$ is a family of mappings $\theta_{\alpha}: U_{\alpha} \rightarrow V$ such that

(a) $\theta_{\alpha}\left(U_{\alpha}\right)$ is an open set.

(b) $\theta_{\alpha}: U_{\alpha} \rightarrow \theta\left(U_{\alpha}\right)$ is a homeomorphism.

(c) The composition of functions $\theta_{\alpha} \theta_{\beta}^{-1}: \theta_{\beta}\left(U_{\alpha} \cap U_{\beta}\right) \rightarrow \theta_{\alpha}\left(U_{\alpha} \cap U_{\beta}\right)$ is smooth.

The topological space $M$ provided with a smooth $V$-structure is called a smooth V-manifold. Every homeomorphism $\theta \in \mathfrak{J}$ is called a chart or coordinate system. If $M$ has a $V$-structure of class $C^{k}, M$ is a $V$-manifold of class $C^{k}$.

\section{Example.}

Let $H$ be a Hilbert space, every open set $M \subset H$ is a $H$-manifold of class $C^{1}$. If $M$ is provided with the families $\{M\}$ and $\{i\}$, where $i: M \rightarrow H$ is the inclusion, $M$ is a $H$-manifold.

Many other manifolds are obtained thanks to the implicit function theorem.

Proposition 2.3 (Product Manifold). Let $\left\{\left(U_{\alpha}, \theta_{\alpha}\right)\right\}$ be a $V_{1}$-structure of class $C^{1}$ on $M_{1}$ and $\left\{\left(U_{i}^{\prime}, \psi_{i}\right)\right\}$ be a $V_{2}$-structure of class $C^{1}$ on $M_{2}$. The collections $\left\{U_{\alpha} \times U_{i}^{\prime}\right\}$ and $\left\{\theta_{\alpha} \times U_{i}^{\prime} \rightarrow V_{1} \times V_{2}\right\}$ give a $V_{1} \times V_{2}$-structure on $M_{1} \times M_{2}$. Therefore, $M_{1} \times M_{2}$ is a $V_{1} \times V_{2}$ manifold of class $C^{1}$.

The next result claims that a level set is a $C^{1}$-manifold.

Theorem 2.28 (Ljusternick [LS34]). Let $E, F$ be real Banach spaces and $G: D(G) \subseteq$ $E \rightarrow F$. Set $M=\{u \in D(G) ; G(u)=0\}$. Assume that $M$ is non empty. If $D(G)$ is open and $G: D(G) \subseteq E \rightarrow F$ is a submersion for all $u \in M$, then $M$ is a manifold of class $C^{1}$. 
Definition 2.19. Let $E$ be a real Banach space and $M$ be a $V$-manifold of class $C^{1}$, we call $M$ a sphere-like if there exists a diffeomorphism $g$ of $M$ onto the unit sphere $S(E)$ of a Banach space E.

Naturally this definition presumes that $S(E)$ is itself a $E$-manifold of class $C^{1}$.

Theorem 2.29. Let $E$ be a real Banach space with norm $q(x):=\|x\|_{E}$ in $C^{1}(E \backslash\{0\})$, $f: E \rightarrow \mathbb{R}$ a $C^{1}$ function on $E$. Then the level surface

$$
M_{c}=\{x \in E ; \quad f(x)=c\}
$$

of $f$ is sphere-like, if

1. $f^{\prime}(x)[x]=\left\langle f^{\prime}(x), x\right\rangle \neq 0$, for all $x \in M_{c}$.

2. Every ray from the origin intersects $M_{c}$ at exactly one point.

The diffeomorphism $r_{c}$ which maps $M_{c}$ on $S(E)$ is given by the restriction to $M_{c}$ of the mapping

$$
\begin{aligned}
r: E \backslash\{0\} & \longrightarrow S(E) \\
x & \longmapsto r(x)=\frac{1}{\|x\|_{E}} x
\end{aligned}
$$

and satifies

$$
\left\|r_{c}^{\prime}(x)\right\|_{E^{\prime}} \leq \frac{2}{\|x\|_{E}}, \text { for all } x \in M_{c}
$$

\subsection{Critical Point Theory}

The problems of extremes with restrictions are generally approached with the techniques that seek critical points of a functional defined on a Banach space with a restriction condition. In this section we explain the necessary conditions for such functional to have critical points, and we present theorems that help to achieve these critical points. More details can be seen in O. Kavian [Kav93], E. Zeidler [Zei84], P. Blanchard and E. Brüning [BB92].

\subsubsection{Critical Points and The Palais-Smale Condition}

Definition 2.20. Let $E$ be a Banach space and let $J: E \rightarrow \mathbb{R}$ be a functional. For $c \in \mathbb{R}$, we define

$$
J^{c}:=\{x \in E ; J(x) \leq c\} .
$$

The sets $J^{c}$ are called the sublevel sets of $J$, or simply sublevels of $J$. Any point $u \in J^{-1}(c)$ is said to be a point at level c. 
Definition 2.21. Let $E$ be a Banach space, $U$ be a open set of $E$ and $J \in C^{1}(U, \mathbb{R})$. The point $u \in U$ is a critical point of $J$ if $J^{\prime}(u)=0$, and $c \in \mathbb{R}$ is a critical value of $J$ if $J^{-1}(c)$ contains a critical point. The set of critical points at the level $c$ is denoted by

$$
K_{c}:=\left\{x \in E ; \quad J(x)=c \text { and } J^{\prime}(x)=0\right\} .
$$

In the sequel we will be interested in topological properties of the sublevel sets of a functional and, mostly, in the correlation between topological properties of sublevels and presence of critical points.

Definition 2.22. Let $E$ a Banach space and let $J: E \rightarrow \mathbb{R}$ be a differentiable functional. A sequence $\left(u_{k}\right) \subset E$ such that the sequence $\left(J\left(u_{k}\right)\right)$ is bounded and $J^{\prime}\left(u_{k}\right) \rightarrow 0$ (in $\left.E^{\prime}\right)$ as $k \rightarrow \infty$, is called a Palais-Smale sequence for $J$.

Let $c \in \mathbb{R}$. If $J\left(u_{k}\right) \rightarrow c$ and $J^{\prime}\left(u_{k}\right) \rightarrow 0$ (in $\left.E^{\prime}\right)$ as $k \rightarrow \infty$, then $\left(u_{k}\right)$ is called a Palais-Smale sequence for $J$ at level $c$. In this case, $c$ is called a Palais-Smale level for $J$.

Considering that we will deal with Hilbert spaces in our work, we need the following definition.

Let $H$ be a Hilbert space, $U \subseteq H$ an open set and $R: H^{\prime} \rightarrow H$ the Riesz isomorphism. Assume that the functional $J: U \rightarrow \mathbb{R}$ is differentiable at $u$. The element $R J^{\prime}(u) \in H$ is called the gradient of $J$ at $u$ and is denoted by $\nabla J(u)$.

Therefore,

$$
J^{\prime}(u)[z]=(\nabla J(u), z)_{H}, \text { for all } z \in H,
$$

where $J^{\prime}(u) \in \mathcal{L}(H, \mathbb{R})=H^{\prime}$.

In this way, the second property of the Palais-Smale sequence implies $\nabla J\left(u_{k}\right) \rightarrow 0$ in $H$ as $k \rightarrow \infty$. We point out that the convergence takes place in the strong topology of $H$.

Definition 2.23. Let $E$ be a Banach space and let $J: E \rightarrow \mathbb{R}$ be a differentiable functional. We say that $J$ satisfies the Palais-Smale condition (shortly: $J$ satisfies $(P S)$ ) if every PalaisSmale sequence for $J$ has a convergent subsequence. We say that $J$ satisfies the Palais-Smale condition at level $c \in \mathbb{R}$ (shortly: $J$ satisfies $(P S)_{c}$ ) if every Palais-Smale sequence at level $c$ has a convergent subsequence.

Proposition 2.4. Let $E$ be a Banach space and let $J: E \rightarrow \mathbb{R}$ be a $C^{1}$-functional. If there exists a Palais-Smale sequence for $J$ and $J$ satisfies (PS), then $J$ has a critical point. If there exists a Palais-Smale sequence for $J$ at level $c$ and $J$ satisfies $(P S)_{c}$, then $J$ has a critical point at level $c$. 


\subsubsection{Critical points under constraints and The Palais-Smale Con- dition}

The next result is a generalization of the existence of Lagrange multipliers for a smooth constraint condition due to L. Ljusternik and L. Schnirelman [LS34].

Theorem 2.30 (Lagrange Multipliers Theorem). Let E a real Banach space and $U\left(u_{0}\right)$ be open neighborhood of $u_{0}$ in E. Suppose that the following two conditions hold.

1. $F: E \rightarrow \mathbb{R}$ is a Frechét differentiable function at $u_{0}$.

2. $G: U\left(u_{0}\right) \rightarrow \mathbb{R}$ is a submersion at $u_{0}$.

Then, if $F$ has a local minimum at $u_{0}$ in $M=\left\{u \in U\left(u_{0}\right) ; G(u)=0\right\}$, there exits $\Lambda$ in $\mathbb{R}$ such that

$$
F^{\prime}\left(u_{0}\right)[x]-\Lambda G^{\prime}\left(u_{0}\right)[x]=0 \text { for all } x \in E .
$$

In view of the Lagrange Multipliers Theorem 2.30 the (PS) condition also takes some adaptations for critical points with constrictions.

Definition 2.24 (Local Palais-Smale Condition). Let $E$ be real Banach space, $J$ a differentiable functional on $E$ and $G: E \rightarrow \mathbb{R}$. Set $M=\{u \in E ; G(u)=0\}$. Assume that $G$ is a differentiable functional on $E$ and $G^{\prime}(u) \neq 0$, for all $u \in M$. Let $c$ be a real number. We say that the restricted functional $\left.J\right|_{M}$ verify the Palais-Smale condition on $M$ if all sequence $\left(u_{n}, \lambda_{n}\right) \in M \times \mathbb{R}$ such that $J\left(u_{n}\right) \rightarrow c$ on $\mathbb{R}$ and $J^{\prime}\left(u_{n}\right)-\lambda_{n} G^{\prime}\left(u_{n}\right) \rightarrow 0$ on $E^{\prime}$ as $n \rightarrow \infty$, contains a subsequence $\left(u_{n_{k}}, \lambda_{n_{k}}\right)$ converging to $(u, \lambda) \in M \times \mathbb{R}$.

Note that Proposition 2.4 is applicable with the Palais-Smale condition with constraint condition.

Theorem 2.31. Assume $H$ is a real infinite dimensional Hilbert space, $f \in C^{1}(H, \mathbb{R})$ is even, $\left.f\right|_{\mathbb{S}_{r}}$ satisfies $(P S)$ and is bounded from below, where $\mathbb{S}_{r}:=\{x \in H ;\|x\|=1\}$. Then, $\left.f\right|_{\mathbb{S}_{r}}$ has infinitely many antipodal pairs of critical points.

\subsubsection{Deformations}

Other implications of the Palais-Smale conditions appears when we work with deformations.

Definition 2.25. Let $E$ a Banach space and $B \subseteq E$ be a subset. $A$ deformation of $B$ is a continuous function $\eta:[0,1] \times B \longrightarrow B$ such that $\eta(0, u)=u$ for all $u \in B$. 
Definition 2.26. Let $E$ be a Banach space and let $A \subseteq B \subseteq E$. We say that $B$ is deformable in $A$ if there exists a deformation $\eta$ of $B$ such that $\eta(t, u) \in A$ for all $u \in A$ and all $t \in[0,1]$, and $\eta(1, u) \in A$ for all $u \in B$.

Next, let us consider a more general version of the deformation theorem due to Clark [Cla72].

Theorem 2.32. Let $E$ be a Banach space and let $J: E \rightarrow \mathbb{R}$ be a $C^{1}$-functional satisfying $(P S)$. Given $c \in \mathbb{R}$ and an open neighborhood $U$ of $K_{c}$, then there exist $\epsilon>0$ and $\eta \in$ $C([0,1] \times E, E)$ such that

1. $\eta(0, u)=u$ for all $u \in E$ and all $t \in[0,1]$,

2. $\eta(t, u)=u$ for all $u \notin J^{-1}[c-2 \epsilon, c+2 \epsilon]$ and all $t \in[0,1]$,

3. $\eta(t, \cdot): E \longrightarrow E$ is a homeomorphism for all $t \in[0,1]$,

4. $J(\eta(t, u)) \leq J(u)$ for all $u \in E$ and all $t \in[0,1]$,

5. $\eta\left(1, J^{c+\epsilon} \backslash U\right) \subset J^{c-\epsilon}$,

6. If $K_{c}=\emptyset, \eta\left(1, J^{c+\epsilon} \backslash U\right) \subset J^{c-\epsilon}$,

7. If $J$ is even, $\eta(t, \cdot)$ is odd in $u$.

In order to apply the deformation properties to certain sets, it is necessary to make use of the following property.

Proposition 2.5. Let $E$ be an $m$-dimensional normed space and $U^{m-1}:=\{u \in E ; \quad\|u\|=1\}$ be a level set. There exists an odd homeomorphism between the sphere $\mathbb{S}^{m-1}$ and the level set $U^{m-1}$.

Proof. Let $\left\{u_{1}, \ldots, u_{m}\right\}$ be a basis of unitary vectors of $E$. For each $u \in U^{m-1}$, there exist unique numbers $x_{1}, \ldots, x_{m}$, not all null, such that $u=x_{1} u_{1}+\cdots+x_{m} u_{m}$. Consider the next functions

$$
\begin{aligned}
& h: U^{m-1} \longrightarrow \mathbb{S}^{m-1} \\
& u \longmapsto h(u)=\frac{x}{\|x\|_{\mathbb{R}^{m}}}, \quad \text { where } x=\left(x_{1}, \ldots, x_{m}\right) \in \mathbb{R}^{m},
\end{aligned}
$$

and

$$
\begin{aligned}
g: \quad \mathbb{S}^{m-1} & \longrightarrow U^{m-1} \\
\left(y_{1}, \ldots, y_{m}\right) & \longmapsto g\left(y_{1}, \ldots, y_{m}\right)=\frac{y_{1} u_{1}+\cdots+y_{m} u_{m}}{\left\|y_{1} u_{1}+\cdots+y_{m} u_{m}\right\|} .
\end{aligned}
$$

It is straight to verify that $h$ is the inverse function of $g$. Thus, $g$ is a bijective function. 
We continue by showing the continuity of $g$. Let $a=\left(a_{1}, \ldots, a_{m}\right)$ and $y=\left(y_{1}, \ldots, y_{m}\right)$ belong to $\mathbb{S}^{m-1}$, consider

$$
\left(y_{1}, \ldots, y_{m}\right) \longmapsto g(y)=\frac{y_{1} u_{1}+\cdots+y_{m} u_{m}}{\left\|y_{1} u_{1}+\cdots+y_{m} u_{m}\right\|}:=\frac{u}{\|u\|}
$$

and

$$
\left(a_{1}, \ldots, a_{m}\right) \longmapsto g(a)=\frac{a_{1} u_{1}+\cdots+a_{m} u_{m}}{\left\|a_{1} u_{1}+\cdots+a_{m} u_{m}\right\|}:=\frac{v}{\|v\|} .
$$

We are going to estimate the norm $\|g(y)-g(a)\|$

$$
\|g(y)-g(a)\|=\left\|\frac{u}{\|u\|}-\frac{v}{\|v\|}\right\|=\left\|\frac{u}{\|u\|}-\frac{u}{\|v\|}+\frac{u}{\|v\|}-\frac{v}{\|v\|}\right\| .
$$

Applying the triangular inequality, we obtain

$$
\begin{aligned}
\|g(y)-g(a)\| & \leq\left\|\frac{u}{\|u\|}-\frac{u}{\|v\|}\right\|+\left\|\frac{u}{\|v\|}-\frac{v}{\|v\|}\right\| \\
& =\left|\frac{1}{\|u\|}-\frac{1}{\|v\|}\right|\|u\|+\frac{1}{\|v\|}\|u-v\| \\
& =\left|\frac{\|v\|-\|u\|}{\|u\|\|v\|}\right|\|u\|+\frac{1}{\|v\|}\|u-v\| \\
& =\frac{1}{\|v\|}|\|v\|-\|u\||+\frac{1}{\|v\|}\|u-v\| .
\end{aligned}
$$

Since

$$
|\|v\|-\|u\|| \leq\|u-v\| \leq \sum_{i=1}^{m}\left|y_{i}-a_{i}\right|\left\|u_{i}\right\|=\sum_{i=1}^{m}\left|y_{i}-a_{i}\right| \leq m\|y-a\|_{\mathbb{R}^{m}}
$$

we obtain that

$$
\|g(y)-g(a)\| \leq \frac{2 m}{\|v\|}\|y-a\|_{\mathbb{R}^{m}}
$$

From above, for every $\epsilon>0$ there exists $\delta=\frac{\epsilon\|v\|}{2 m}>0$ such that for all $y$, if $\|y-a\|_{\mathbb{R}^{m}}<\delta$, then $\|g(y)-g(a)\|<\epsilon$. Since $a \in \mathbb{S}^{m-1}$ is an arbitrary point, $g$ is a continuous function on $\mathbb{S}^{m-1}$.

Now, the sphere $\mathbb{S}^{m-1}$ is a compact set and $g$ is a bijective continuous function, then $g$ is a homeomorphism.

Finally,

$$
g(-y)=\|-u\|^{-1}(-u)=-\|u\|^{-1}(u)=-g(y),
$$

that is, $g$ is an odd homeomorphism between $\mathbb{S}^{m-1}$ and $U^{m-1}$.

Now, we have the necessary background to present a characterization for the critical 
points of a differentiable functional; the critical points are characterized by the genus of certain sets. This result was given by of Ambrosetti, A. and Rabinowitz, P. H. in [AR73].

Theorem 2.33. Let $E$ be a Banach space, $B_{r}=\{u \in E ;\|u\|<r\}$,

$$
\Gamma_{m}:=\{K \subset E ; \quad K \text { is symmetric and closed with } \gamma(K) \geq m\}
$$

and let $J \in C^{1}(E, \mathbb{R})$ be a functional satisfying:

1. $J(0)=0$.

2. There exists a $\rho>0$ such that $J>0$ in $\partial B_{\rho} \backslash\{0\}$ and $J \geq \alpha>0$ on $\partial B_{\rho}$.

3. J satisfies (PS) condition.

For each $m \in \mathbb{N}$, let

$$
b_{m}=\inf _{K \in \Gamma_{m}} \sup _{u \in K} J(u) .
$$

Then $0<\alpha \leq b_{m} \leq b_{m+1}$ and $b_{m}$ is a critical value of $J$. Moreover, if $b_{m+1}=\ldots=b_{m+r}=b$, then $\gamma\left(K_{b}\right) \geq r$.

\subsection{Trace Theory}

Let $\Omega$ a bounded open set in $\mathbb{R}^{N}$ with boundary $\partial \Omega$. Consider the Dirichlet and Neumann problems respectively

$$
\left\{\begin{aligned}
-\Delta u=f & \text { in } \Omega \\
\left.u\right|_{\partial \Omega}=h & \text { on } \partial \Omega
\end{aligned}\right.
$$

and

$$
\left\{\begin{aligned}
-\Delta u=f & \text { in } \Omega, \\
\left.\frac{\partial u}{\partial \nu}\right|_{\partial \Omega}=h & \text { on } \partial \Omega,
\end{aligned}\right.
$$

where $f$ and $h$ are real valued functions defined on $\Omega$ and $\partial \Omega$ respectively, $\nu=\nu(x)$ is the outward normal to $\partial \Omega$ at $x, \frac{\partial u}{\partial \nu}(x)=\nabla u(x) . \nu(x)$ and $\Delta=\sum_{i=1}^{N} \frac{\partial^{2}}{\partial x_{i}^{2}}$.

If we want to solve the problem (D) in the frame of Sobolev spaces, we need to define the meaning of the constraint condition $\left.u\right|_{\partial \Omega}$ being equal to $h$. Similarly, in the problem $(\mathrm{N})$ we need to give a sense the constraint of $\left.\frac{\partial u}{\partial \nu}\right|_{\partial \Omega}$ is equal to $h$. Thus, in this section we will assign a meaning to expressions like $\left.u\right|_{\partial \Omega}$ and $\left.\frac{\partial u}{\partial \nu}\right|_{\partial \Omega}$ when $u$ belongs to some Sobolev space. Therefore, we require to generalize the notion of boundary values to such functions. The details of the proofs of the next theorems are in S. Kesavan [Kes89].

We will start with the case $\Omega=\mathbb{R}_{+}^{N}$. The case of a smooth open set $\Omega$ will follow by the use of local charts and a corresponding partition of unity. The theory that we present is valid for other values of $p$ but we restrict our attention to the case $p=2$. 
Theorem 2.34. Let $\Omega=\mathbb{R}_{+}^{N}$. Then there exists a continuous linear map $\gamma_{0}: H^{1}\left(\mathbb{R}_{+}^{N}\right) \rightarrow L^{2}\left(\mathbb{R}^{N-1}\right)$ such that, if $v$ is continuous on $\overline{\mathbb{R}_{+}^{N}}$, then

$$
\gamma_{0}(v)=\left.v\right|_{\mathbb{R}^{N-1}}
$$

Definition 2.27. The image of the map $\gamma_{0}$ is called the trace map of order zero.

Theorem 2.35. The image of the map $\gamma_{0}$ is the space $H^{\frac{1}{2}}\left(\mathbb{R}^{N-1}\right)$.

Lemma 2.1 (Green's formula). Let $u, v \in H^{1}\left(\mathbb{R}_{+}^{N}\right)$. Then

$$
\begin{gathered}
\int_{\mathbb{R}_{+}^{N}} u \frac{\partial v}{\partial x_{i}}=-\int_{\mathbb{R}_{+}^{N}} \frac{\partial u}{\partial x_{i}} v \quad \text { for all } \quad 1 \leq i \leq N-1 \\
\int_{\mathbb{R}_{+}^{N}} u \frac{\partial v}{\partial x_{n}}=-\int_{\mathbb{R}_{+}^{N}} \frac{\partial u}{\partial x_{n}} v-\int_{\mathbb{R}_{+}^{N-1}} \gamma_{0}(u) \gamma_{0}(v) .
\end{gathered}
$$

Corollary 2.35.1. If $u, v \in H^{1}\left(\mathbb{R}_{+}^{N}\right)$ and at least one of them is in the $k e r\left(\gamma_{0}\right)$, then equation (2.13) holds for all $1 \leq i \leq N$.

Theorem 2.36. $\operatorname{ker}\left(\gamma_{0}\right)=H_{0}^{1}\left(\mathbb{R}_{+}^{N}\right)$.

Let us now deal with the case of a bounded open set $\Omega$ of class $C^{1}$. Let $\left\{\left(U_{j}, T_{j}\right)\right\}_{j=1}^{k}$ be associated local charts for the boundary $\partial \Omega$ and let $\left\{\psi_{j}\right\}_{j=1}^{k}$ be a partition of unity subordinate to the cover $\left\{U_{j}\right\}_{j=1}^{k}$ of $\partial \Omega$. If $u \in H^{1}(\Omega)$, then $\left(\left.\psi_{j} u\right|_{U_{j} \cap \Omega}\right) \circ T_{j} \in H_{0}^{1}\left(\mathbb{R}_{+}^{N}\right)$ and so we can define its trace as an element of $H^{1 / 2}\left(\mathbb{R}^{N-1}\right)$. Coming back $T_{j}^{-1}$ we can define the trace on $U_{j} \cap \partial \Omega$. Hence, we get the trace $\gamma_{0}(u)$ in $L^{2}(\partial \Omega)$ and the image will be precisely $H^{\frac{1}{2}}(\partial \Omega)$. Similarly, if the boundary is smoother we can define the higher order traces $\gamma_{j}$. In particular we have the following result.

Theorem 2.37. Let $\Omega \subset \mathbb{R}^{N}$ be a bounded open set of class $C^{m+1}$ with boundary $\partial \Omega$. Then there exists a trace map $\gamma=\left(\gamma_{0}, \gamma_{1}, \ldots, \gamma_{m-1}\right)$ from $H^{m}(\Omega)$ into $\left(L^{2}(\Omega)\right)^{m}$ such that

1. If $v \in C^{\infty}(\bar{\Omega})$, then $\gamma_{0}(v)=\left.v\right|_{\partial \Omega}, \gamma_{1}(v)=\left.\frac{\partial v}{\partial \nu}\right|_{\partial \Omega}, \ldots$, and $\gamma_{m-1}(v)=\left.\frac{\partial^{m-1} v}{\partial \nu^{m-1}}\right|_{\partial \Omega}$, where $\nu$ is the unit exterior normal to the boundary $\partial \Omega$.

2. The image of $\gamma$ is the space

$$
\prod_{j=0}^{m-1} H^{m-j-\frac{1}{2}}(\partial \Omega)
$$

3. The kernel of $\gamma$ is $H_{0}^{m}(\Omega)$. 
The trace theorem above helps us to obtain Green's Theorem for functions in $H^{1}(\Omega)$, where $\Omega$ of class $C^{1}$. If $\nu(x)$ denotes the unit exterior normal vector on the boundary $\partial \Omega$, we denote its components along the coordinate axes by $\nu_{j}(x)$. Thus, we write $\nu=\left(\nu_{1}, \ldots, \nu_{N}\right)$.

Theorem 2.38 (Green's Theorem). Let $\Omega$ be a bounded open set of $\mathbb{R}^{N}$ of class $C^{1}$ lying in the same side of its boundary $\partial \Omega$. Then

$$
\int_{\Omega} u \frac{\partial v}{\partial x_{i}}=-\int_{\Omega} \frac{\partial u}{\partial x_{i}}+\int_{\partial \Omega}\left(\gamma_{0} u\right)\left(\gamma_{0} v\right) \nu_{i} \text { for all } u, v \in H^{1}(\Omega), \quad i=1, \ldots, N,
$$

and

$$
-\int_{\Omega} \Delta u v d x=\sum_{i=1}^{N} \int_{\Omega} \frac{\partial u}{\partial x_{i}} \frac{\partial v}{\partial x_{i}} d x-\int_{\partial \Omega}\left(\gamma_{1} u\right)\left(\gamma_{0} v\right) d \partial \Omega \text { for all } u \in H^{2}(\Omega), v \in H^{1}(\Omega) \text {. }
$$




\section{Chapter 3}

\section{Some Physical Facts}

In this chapter, we describe the system of equations Schrödinger-Bopp-Podolsky from the point of view of physics. We present the physical phenomena that the equations are modeling in the three dimensional space. For this purpose, only in this chapter $N=3$. Also, we will define some terms that we will use in next chapters.

\subsection{Variational Principle}

According with I. Ekeland [Eke74] the variational principle states that if a differentiable functional $F$ attains its minimum at some point $u$, then $F^{\prime}(u)=0$. Therefore, most of the times, solving differential equations is concerned with finding extremal points of a certain functional whose domain is a space of curves. It is worth mentioning that almost all the fundamental equations of motion obey a variational principle.

Generally, the goal of the variational principle is to minimize a definite integral of the form

$$
J(y)=\int_{a}^{b} f\left(x, y(x), y^{\prime}(x)\right) d x
$$

subject to the boundary conditions $y(a)=y_{a}$ and $y(b)=y_{b}$. We emphasize that $J$ is a Fréchet differentiable functional whose domain is a space of curves. The differential of that functional is also called its variation, and equaling the differential to zero, we obtain the Euler-Lagrange equation relative to $J$. An extremal of the functional $J$ is a curve $y_{0}$ such that $J^{\prime}\left(y_{0}\right)=0$.

An example of such functional arises when we look for the length of a curve. Indeed, let $\gamma=\left\{(t, x): x=x(t), t_{0} \leq t \leq t_{1}\right\}$ be a curve in the plane, $x^{\prime}=\frac{d x}{d t}$ and $L=L\left(t, x(t), x^{\prime}(t)\right)$ a differentiable function. We define the functional $\Phi$ by

$$
\Phi(\gamma)=\int_{t_{0}}^{t_{1}} L\left(t, x(t), x^{\prime}(t)\right) d t
$$


We get the length of $\gamma$ by defining $L=\sqrt{1+\left[x^{\prime}(t)\right]^{2}}$.

The following is an example of the variational principle extracted from physics. The equations of motion of $k$ particles whose positions at the time $t$ are given by the functions $x_{j}(t), j=1, \ldots, k$, are the Euler-Lagrange equations relative to the functional

$$
\mathcal{S}=\int\left(\sum_{j=1}^{k} \frac{m_{j}}{2}\left|x_{j}^{\prime}(t)\right|^{2}-V\left(t, x_{1}(t), \ldots, x_{k}(t)\right)\right) d t
$$

where $m_{j}$ is the mass of the $j$-th particle and $V$ is the potential energy of the system.

Also, physics describes the motion of a particle in the next principle.

Theorem 3.1 (Hamilton's principle of least action). Motions of the mechanical system coincide with extremes of the functional

$$
\Phi(\gamma)=\int_{\Omega} L d t
$$

where $L=T-U$ is the difference between the kinetic and potential energy, the set $\gamma=\left\{(t, x): x=x(t), t_{0} \leq t \leq t_{1}\right\}$ is a differentiable curve, $x^{\prime}=\frac{d x}{d t}$ and $L=L\left(t, x(t), x^{\prime}(t)\right)$ a differentiable function.

\subsection{Eigenvalues and eigenfunctions of the Schrödinger equation}

In this section, we explain the presence of the Schrödinger equation in system (1).

The Schrödinger equation describes the wave equation that satisfies a Broglie particle, i. e. a particle that share both wave and particle properties.

We denote the kinetic energy by $\mathrm{E}$ and the light frequency by $\omega$. These variables are related by the Plank constant $\hbar$

$$
\mathrm{E}=\hbar \omega .
$$

On one hand, the Plank constant defines the momentum vector

$$
\mathbf{p}=\hbar \mathbf{k}
$$

where $\mathbf{k}$ is called the wave number $\mathbf{k}$, in the special case of a matter wave, $|\mathbf{k}|$ is given by $|\mathbf{k}|=\frac{2 \pi}{\lambda}$, where $\lambda$ is the wavelength. On the other hand, the speed of light in vacuum, denoted by $c$, defines the angular frequency of the wave $\omega$ as follows

$$
\omega=c|\mathbf{k}|
$$


Consider a wave

$$
\psi(t, x)=\exp (-i(\omega t-\mathbf{k} \cdot x))
$$

where $x \in \mathbb{R}^{3}$. The differentiation respect to $t$ is

$$
\frac{\partial \psi}{\partial t}(t, x)=-i \omega \exp (-i(\omega t-\mathbf{k} \cdot x))
$$

multiplying by $i \hbar$ and replacing (3.1) we obtain

$$
i \hbar \frac{\partial \psi}{\partial t}(t, x)=\hbar \omega \psi(t, x)=\mathrm{E} \psi(t, x) .
$$

Also, the differentiation of $\psi$ respect to $x$ gives

$$
\nabla \psi(t, x)=i \mathbf{k} \psi(t, x)
$$

by Equation (3.2) we have

$$
\hbar \nabla \psi(t, x)=i \mathbf{p} \psi(t, x),
$$

hence, the momentum turns out to be

$$
\mathbf{p}=\frac{-i \hbar \nabla \psi(t, x)}{\psi(t, x)}
$$

The total energy of a particle with mass $m$ and potential energy $v(x)$ is given by

$$
\mathrm{E}=\frac{|\mathbf{p}|^{2}}{2 m}+v(x)
$$

Observe that multiplying $\mathrm{E}$ by $\psi(t, x)$ we have another expression for the equality (3.4), i. e.

$$
i \hbar \frac{\partial \psi}{\partial t}(t, x)=\left[\frac{|\mathbf{p}|^{2}}{2 m}+v(x)\right] \psi(t, x) .
$$

Replacing the momentum $\mathbf{p}$ in (3.5) we finally obtain the Schrödinger equation

$$
i \hbar \frac{\partial \psi}{\partial t}(t, x)=-\frac{\hbar^{2}}{2 m} \nabla^{2} \psi(t, x)+v(x) \psi(t, x)
$$

where $\nabla^{2} \psi(t, x)=\Delta \psi(t, x)=\sum_{i=1}^{3} \frac{\partial^{2} \psi(t, x)}{\partial x_{i}^{2}}$.

In order to find solutions for the Schrödinger equation (3.6), we try a solution of the form

$$
\psi(t, x)=T(t) u(x)
$$

where $T:[0, \infty) \rightarrow \mathbb{R}$ is positive and independent of $x$ and $u: \mathbb{R}^{3} \rightarrow \mathbb{R}$ is independent of $t$. 
From equation above follows that

$$
\nabla^{2} \psi(t, x)=T(x) \nabla^{2} u(x)
$$

and

$$
\frac{\partial \psi}{\partial t}(x, t)=u(x, t) \frac{d T}{d t} .
$$

Exchanging these equalities in the Schrödinger equation, we obtain

$$
i \hbar u(x) \frac{d T}{d t}=-\frac{\hbar^{2}}{2 m} T(t) \nabla^{2} u(x)+v(x) T(t) u(x),
$$

hence

$$
\frac{i \hbar \frac{d T}{d t}}{T(t)}=\frac{-\frac{\hbar^{2} \nabla^{2} u(x)}{2 m}+v(x) u(x)}{u(x)} .
$$

Since the right-hand side of the equality above only depends on $x$ and the left-hand side term only depends on $t$, the expressions on each side of the equation must be equal to a constant term. Such constant will be denoted by $\omega^{1}$. Thus, (3.8) implies the two ordinary differential equations

$$
\left\{\begin{aligned}
i \hbar \frac{d T}{d t} & =\omega T(t), \\
-\frac{\hbar^{2} \nabla^{2} u(x)}{2 m}+v(x) u(x) & =\omega u(x) .
\end{aligned}\right.
$$

Determining the values that may be assigned to $\omega$ depends on the boundary conditions. Then, we are facing the problem of not only solving (3.9) but finding the general solution $u(x)=u_{\omega}(x)$ for the required conditions. It can be shown that there is only a discrete set of values of $\omega$. Any such value $\omega_{n}$ is called eigenvalue. The corresponding solution $u_{\omega}$ is called eigenfunction.

Observe that both of the differential equations in (3.9) are eigenvalue problems. Furthermore, the product of an eigenfunction by a nonzero constant is also an eigenfunction corresponding to the same eigenvalue. For this reason, we may impose the normalizing condition

$$
\int_{\Omega} u^{2}=1
$$

for every function that we deal with. Thus, the Schrödinger equation is equivalent to a Lagrange multiplier problem. Note that the normalizing condition is also included in our system Schrödinger-Bopp-Podolsky, see end of the Chapter 1.

Finally, note that the Schrödinger equation (3.6) coincides with the first equation of our principal Schrödinger-Bopp-Podolsky system if $m=\frac{\hbar^{2}}{2}, v$ is the potential $\phi$, and $\psi$ is the function $u$.

\footnotetext{
${ }^{1}$ Using the energy equation, the constant $\omega$ can be showed to be $\mathrm{E}$ which depends on the boundary conditions.
} 


\subsection{Description of the solution of the Schrödinger equa- tion}

We recall the Schrödinger equation in (3.6)

$$
i \hbar \frac{\partial \psi}{\partial t}(t, x)=-\frac{\hbar^{2}}{2 m} \nabla^{2} \psi(t, x)+v(x) \psi(t, x),
$$

and the system (3.9) with $\omega=\mathrm{E}$

$$
\left\{\begin{aligned}
i \hbar \frac{d T}{d t} & =\mathrm{E} T(t) \\
-\frac{\hbar^{2} \nabla^{2} u(x)}{2 m}+v(x) u(x) & =\mathrm{E} u(x) .
\end{aligned}\right.
$$

In this section, we will solve that system of equations.

By the method of integrating factors in the first equation, we obtain

$$
T(t)=\exp \left(\frac{-i \mathrm{E}}{\hbar} t\right)
$$

Since we are looking for a function $\psi$ as in Equation (3.7), we have

$$
\psi(t, x)=\exp \left(\frac{-i \mathrm{E}}{\hbar} t\right) u(x)
$$

Note that $\psi(0, x)=u(x)$.

For the second equation of the system above we consider a particle with potential $v(x)=$ 0 , then we obtain

$$
\frac{d^{2} u(x)}{d x^{2}}+\frac{2 m \mathrm{E}}{\hbar^{2}} u(x)=0
$$

where $u(0)=0$.

The solutions of equation (3.10) depend of the energy value E. Indeed, if $\mathrm{E}=0$ we have an homogeneous differential equation, then integrating we have

$$
u(x)=A+B x
$$

where $A$ and $B$ are constants. If $\mathrm{E} \neq 0$, the characteristic equation of the differential equation (3.10) can have:

1. two complex roots if the energy $\mathrm{E}$ is negative then

$$
u(x)=A \cosh \left(\frac{\sqrt{2 m|\mathrm{E}|}}{\hbar} x\right)+B \sinh \left(\frac{\sqrt{2 m|\mathrm{E}|}}{\hbar} x\right)
$$


2. two real roots if the energy $\mathrm{E}$ is positive then

$$
u(x)=A \cos \left(\frac{\sqrt{2 m \mathrm{E}}}{\hbar} x\right)+B \sin \left(\frac{\sqrt{2 m \mathrm{E}}}{\hbar} x\right)
$$

where $A$ and $B$ are constants.

The condition $u(0)=0$ implies in every case that $A=0$. When $\mathrm{E}<0$, it also implies that $B=0$. Hence, we get the trivial solution. But, in the case $\mathrm{E}>0$, we obtain a non-null solution $u(x)=B \sin \left(\frac{\sqrt{2 m E}}{\hbar} x\right)$ whenever $\mathrm{B}$ is non-null. The value of $B$ depends on the normalization condition.

Taking

$$
\frac{\sqrt{2 m \mathrm{E}}}{\hbar}=\frac{n \pi}{a_{n}}
$$

where $n$ and $a_{n}$ are positive integers. Then, the energy take the following discrete values

$$
\mathrm{E}=\mathrm{E}_{n}=\frac{n^{2} \pi^{2} \hbar^{2}}{2 m a_{n}^{2}}
$$

Thereby, we have discrete solutions $u(x)=u_{n}(x)=B \sin \left(\frac{n \pi x}{a_{n}}\right)$.

From equation (3.1) and the discrete values of energy, we obtain that $\omega_{n}=\frac{n^{2} \pi^{2} \hbar}{2 m a^{2}}$. Observe that the sequence $\omega_{n} \rightarrow \infty$ as $n \rightarrow \infty$.

We will obtain similar results in Chapter 4.

\subsection{Non-linear Electrodynamics}

This section attends to the equations of system (1) as equations that obey a variational principle.

The dynamics of electrical and magnetic fields are well described by the Maxwell equations, then those fields satisfy the linear superposition principle. This principle applies to phenomena at the macroscopic level and at the atomic level. But at the subatomic level, there could be a non-linear behavior because as charged particles approach each other, their electrical fields increase rapidly. The study of this phenomenon is called electrodynamic generalizations, also called non-linear electrodynamics (NLED), that proposes to replace the lagrangian of Maxwell for another one containing non-linear terms of electromagnetic invariants.

In separated studies, F. Bopp [Bop40] and B. Podolsky [Pod42] proposed a theory of second order gauge invariance to solve the infinity problem that appears in the classical Maxwell theory. In classical theory, the electric potential $\phi$ of some charge distribution, 
whose density is $\rho$, satisfies the equation

$$
\Delta \phi=\rho \text { in } \mathbb{R}^{3}
$$

If the differential equation above has constant coefficient $\rho=4 \pi \delta_{x_{0}}, \quad x_{0} \in \mathbb{R}^{3}$, its fundamental solution is $g\left(x-x_{0}\right)$ where $g(x)=\frac{1}{|x|}$. Thus the electrostatic energy is

$$
E_{M a x}(g)=\frac{1}{2} \int|\nabla g|^{2}=+\infty
$$

The Bopp-Podolsky theory replaces the Posisson equation (3.11) by

$$
-\Delta \phi+a^{2} \Delta^{2} \phi=\rho
$$

If $\rho=4 \pi \delta_{x_{0}}$, we have an operator $-\Delta+a^{2} \Delta^{2}$ with constant coefficients, whose fundamental solution is given by

$$
K(x)=\frac{1-\exp \left(\frac{-|x|}{a}\right)}{|x|} .
$$

Note that the function $K$ has no singularity in $x_{0}$ since it satisfies

$$
\lim _{x \rightarrow x_{0}} K\left(x-x_{0}\right)=\frac{1}{a}
$$

and its energy is

$$
E_{B P}=\frac{1}{2} \int_{\mathbb{R}^{3}}|\nabla K|^{2}+\frac{a^{2}}{2} \int_{\mathbb{R}^{3}}|\Delta K|^{2}<\infty .
$$

P. d'Avenia and G. Siciliano show in [dS19] that $K$ is the fundamental solution of the operator $-\Delta+a^{2} \Delta^{2}$.

\subsubsection{A system of Schr̈odinger-Bopp-Podolsky equations}

By the Hamilton principle, every motion equation obeys to an Euler-Lagrange equation. We present the Euler-Lagrange equations for a system of equations of Schrodinger and Bopp-Podolsky.

The Schrödinger equation for a particle in an electrodynamic field, whose gauge potentials are $\mathbf{A}$ and $\phi$, is

$$
i \hbar \frac{\partial \psi}{\partial t}=\frac{1}{2 m}\left(-i \hbar \nabla-\frac{e}{c} \mathbf{A}\right)^{2} \psi-e \phi \psi
$$

where $\psi(t, x) \in \mathbb{C}$ is the wave function, $m$ is the mass, $e$ is the charge of the particle and $\hbar$ is the Plank constant. The Lagrangian density relative to (3.13) is given by

$$
\mathcal{L}_{S c}=\frac{1}{2}\left[i \hbar \frac{\partial \psi}{\partial t} \bar{\psi}+e \phi|\psi|^{2}-\frac{1}{2 m}\left|\left(-i \hbar \nabla-\frac{e}{c} \mathbf{A}\right) \psi\right|^{2}\right] .
$$


If we set $\psi(x, t)=u(x, t) \exp \left(\frac{i S(x, t)}{\hbar}\right), \mathcal{L}_{S c}$ takes the form

$$
\mathcal{L}_{S c}=\frac{\hbar^{2}}{2 m}|\nabla u|^{2}-\left[S_{t}-e \phi+\frac{1}{2 m}\left(\nabla S-\frac{e}{c} \mathbf{A}\right)^{2}\right] u^{2}
$$

where $\phi: \mathbb{R}^{3} \rightarrow \mathbb{R}$ and $\mathbf{A}: \mathbb{R}^{3} \rightarrow \mathbb{R}^{3}$. The pair of functions $(\phi, \mathbf{A})$ is the gauge potential of the electromagnetic field $(\mathbf{E}, \mathbf{H})$ and satisfies

$$
\mathbf{E}=-\nabla \phi-\frac{1}{c} \partial_{t} \mathbf{A} \quad \text { and } \quad \mathbf{H}=\nabla \times \mathbf{A} .
$$

B. Podolsky obtained in [Pod42] that the Bopp-Podolsky lagrangian density is

$$
\begin{aligned}
\mathcal{L}_{B P}= & \frac{1}{8 \pi}\left\{|\mathbf{E}|^{2}-|\mathbf{H}|^{2}+a^{2}\left[(\operatorname{div} \mathbf{E})^{2}-\left|\nabla \times \mathbf{H}-\frac{1}{c} \partial_{t} \mathbf{E}\right|^{2}\right]\right\} \\
= & \frac{1}{8 \pi}\left\{\left|\nabla \phi+\frac{1}{c} \partial_{t} \mathbf{A}\right|^{2}-|\nabla \times \mathbf{A}|^{2}+a^{2}\left[\left(\Delta \phi+\frac{1}{c} \operatorname{div} \partial_{t} \mathbf{A}\right)^{2}\right.\right. \\
& \left.\left.-\left|\nabla \times \nabla \times \mathbf{A}+\frac{1}{c} \partial_{t}\left(\nabla \phi+\frac{1}{c} \partial_{t} \mathbf{A}\right)\right|^{2}\right]\right\} .
\end{aligned}
$$

Thus, the total action is

$$
\mathcal{S}(\psi, \phi, \mathbf{A})=\int \mathcal{L}_{S c}+\mathcal{L}_{B P} d x d t
$$

Considering $\psi(t, x)=\exp (i S(t, x)) u(t, x)$, the Euler-Lagrange equations of $\mathcal{S}$ are given by

$$
\begin{aligned}
0= & \frac{\hbar^{2}}{2 m} \Delta u+\left[S_{t}-e \phi+\frac{1}{2 m}\left(\nabla S-\frac{e}{c} \mathbf{A}\right)^{2}\right] u, \\
0= & \frac{\partial}{\partial t}\left(u^{2}\right)-\frac{1}{m} \operatorname{div}\left[\left(\nabla S-\frac{e}{c} \mathbf{A}\right) u^{2}\right], \\
4 \pi e u^{2}= & a^{2}\left[\Delta\left(\Delta \phi+\frac{1}{c} \operatorname{div} \partial_{t} \mathbf{A}\right)-\frac{1}{c} \partial_{t} \operatorname{div}\left(\nabla \times \nabla \times \mathbf{A}+\frac{1}{c} \partial_{t}\left(\nabla \phi+\frac{1}{c} \partial \mathbf{A}\right)\right)\right] \\
& -\nabla \cdot\left(\frac{1}{c} \mathbf{A}_{t}+\nabla \phi\right), \\
0= & \frac{\hbar q}{m c}\left(\nabla S-\frac{q}{\hbar c} \mathbf{A}\right) u^{2}-\frac{1}{4 \pi}\left[\frac{1}{c} \partial_{t}\left(\nabla \phi+\frac{1}{c} \partial_{t} \mathbf{A}+\nabla \times \nabla \times \mathbf{A}\right)\right] \\
& +\frac{a^{2}}{4 \pi}\left[\frac{1}{c} \nabla \partial_{t}\left(\Delta \phi+\frac{1}{c} \operatorname{div} \partial_{t} \mathbf{A}\right)-\nabla \times \nabla \times \nabla \times \nabla \times \mathbf{A}-\frac{1}{c^{2}} \partial_{t t} \nabla \times \nabla \times \mathbf{A}\right. \\
& \left.-\frac{1}{c} \nabla \times \nabla \times \partial_{t}\left(\nabla \phi+\frac{1}{c} \partial_{t} \mathbf{A}\right)-\frac{1}{c^{3}} \partial_{t t t}\left(\nabla \phi+\frac{1}{c} \partial_{t} \mathbf{A}\right)\right] .
\end{aligned}
$$

if we look for solutions $u, S, \mathbf{A}$ and $\phi$ for system above of the type

$$
u=u(x), \quad S=\omega t, \quad \mathbf{A}=0 \quad \text { and } \quad \phi=\phi(x),
$$


the first and second equations are satisfied, while the third and fourth equations become the Schrödinger-Bopp-Podolsky equations

$$
\left\{\begin{aligned}
-\Delta u+\phi u & =\omega u & \text { in } \Omega \\
\Delta^{2} \phi-\Delta \phi & =u^{2} & \text { in } \Omega
\end{aligned}\right.
$$

The system above together with the boundary conditions

$$
u=\Delta \phi=\phi=0 \quad \text { on } \quad \partial \Omega
$$

and normalizing condition

$$
\int_{\Omega} u^{2}=1
$$

will be called the SBP system. 


\section{Chapter 4}

\section{Solution of the SBP system}

In this chapter, we study the eigenvalue problem that incorporates the biharmonic operator. We aim to show the existence of real numbers $\omega$ and real functions $u$ and $\phi$ satisfying the SBP system

$$
\left\{\begin{aligned}
-\Delta u+\phi u & =\omega u \\
\Delta^{2} \phi-\Delta \phi & =u^{2}
\end{aligned} \text { in } \Omega\right.
$$

with the boundary conditions

$$
u=\Delta \phi=\phi=0 \quad \text { on } \quad \partial \Omega
$$

and normalizing condition

$$
\int_{\Omega} u^{2}=1
$$

where $\Omega$ is a bounded open subset of $\mathbb{R}^{N}, N \geq 3$.

Let $\Delta$ be the Laplace operator. The biharmonic operator is defined as $\Delta^{2}$, it is a differential operator of forth order given by

$$
\Delta^{2}=\sum_{i=1}^{N} \frac{\partial^{4}}{\partial x_{i}^{4}}+\sum_{\substack{i, j=1 \\ i \neq j}}^{N} \frac{\partial^{4}}{\partial^{2} x_{i} \partial^{2} x_{j}}
$$

\subsection{Description of the solutions}

Defining a weak solution for a differential problem also implies determining the space of functions where that weak solution belongs. First, considering from the SBP system

$$
\left\{\begin{aligned}
-\Delta u+\phi u & =\omega u \text { in } \Omega \\
u & =0 \quad \text { on } \partial \Omega
\end{aligned}\right.
$$


we recognize an homogeneus Dirichlet problem. In order to obtain the definition of a weak solution for the subsystem (4.2), it is necessary to obtain the bilinear form associated with it, see Section 2.5. So, we consider a classical solution $u \in C^{2}(\bar{\Omega})$ that satisfies (4.2) for all $x \in \bar{\Omega}$. Now, taking $v \in C_{0}^{1}(\Omega)$, we multiply the first equation in (4.2) by $v$ and integrating over $\Omega$

$$
-\int_{\Omega} \Delta u v+\int_{\Omega} \phi u v=\int_{\Omega} \omega u v
$$

By the Green's formula (2.10)

$$
\int_{\Omega} \nabla u \nabla v+\int_{\Omega} \phi u v=\int_{\Omega} \omega u v, \quad \text { for all } v \in C_{0}^{1}(\Omega)
$$

This formula also makes sense if we weaken the conditions for the function $u$. Observe that the equation is satisfied by integrable functions $u$ and $v$ whose all first derivatives are also integrable. In particular, if $v=u$

$$
\int_{\Omega}|\nabla u|^{2}+\int_{\Omega} \phi u^{2}=\int_{\Omega} \omega u^{2}
$$

Thus, it is sufficient to let $u$ be a function in $L^{2}(\Omega)$ with compact support and $\nabla u \in L^{2}(\Omega)$. Note that we do not need $\phi$ to be continuous. Hence, we determine that $u$ belongs to the Sobolev space $H_{0}^{1}(\Omega)$ and the continuous bilinear form associated to the equation in (4.2) is

$$
a(u, v):=\int_{\Omega} \nabla u \nabla v+\int_{\Omega} \phi u v, \quad \text { for all } u, v \in H_{0}^{1}(\Omega) .
$$

We have already seen that formulating a weak solution for the SBP system we had used the classical Green's formula, then before working on the second equation of (4.1) we need a formula that generalizes the Green's formula for the powers of the Laplacian operator.

Proposition 4.1. Let $\Omega$ be a smooth open set in $\mathbb{R}^{N}$ lying on the same side of its boundary $\partial \Omega$. Let $u \in H_{0}^{1}(\Omega)$ and $v \in C^{1}(\bar{\Omega}) \cap H^{2}(\Omega)$ such that $\Delta v=v=u=0$ on $\partial \Omega$, then

$$
\int_{\Omega} u\left(\Delta^{2} v\right) d x=\int_{\Omega} \Delta u \Delta v d x
$$

Proof. Since $C^{\infty}(\bar{\Omega})$ is dense in $H_{0}^{1}(\Omega)$ and $H^{2}(\Omega)$, there exist mollifiers sequences $\left\{u_{m}\right\}_{m}$ and $\left\{v_{m}\right\}_{m} \in C^{\infty}(\bar{\Omega})$ converging to $u \in H_{0}^{1}(\Omega)$ and $v \in C^{1}(\bar{\Omega}) \cap H^{2}(\Omega)$ respectively. Keep in mind that

$$
\Delta^{2} v_{m}=\Delta\left(\Delta v_{m}\right), \quad \text { for all } m \in \mathbb{N} .
$$

For every $i, j \in\{1, \ldots, N\}$, we apply the classical Green's formula in Theorem (2.10) to the term $i j$ of the sum $u_{m}\left(\Delta v_{m}\right)$ two times, we obtain 


$$
\begin{aligned}
\int_{\Omega} u_{m}\left(\frac{\partial^{4} v_{m}}{\partial x_{j}^{2} \partial x_{i}^{2}}\right) d x= & -\left[-\int_{\Omega} \frac{\partial^{2} v_{m}}{\partial x_{j}^{2}} \frac{\partial^{2} u_{m}}{\partial x_{i}^{2}} d x+\int_{\partial \Omega} \nu_{i} \frac{\partial u_{m}}{\partial x_{i}} \frac{\partial^{2} v_{m}}{\partial x_{j}^{2}} d \sigma\right] \\
& +\int_{\partial \Omega} u_{m} \frac{\partial^{3} v_{m}}{\partial x_{i} \partial x_{j}^{2}} \nu_{i} d \sigma
\end{aligned}
$$

where $\nu(x)=\left(\nu_{1}(x), \cdots, \nu_{N}(x)\right)$ is the outward normal to $\partial \Omega$ at $x$ and $\sigma$ is the surface measure on $\partial \Omega$.

By Theorem 2.37, there exists a trace map $\gamma=\left(\gamma_{0}, \gamma_{1}\right)$ from $H_{0}^{1}(\Omega)$ into $\left(L^{2}(\Omega)\right)^{2}$ such that

$$
\gamma_{0}\left(u_{m}\right)=\left.u_{m}\right|_{\partial \Omega} \quad \text { and } \quad \gamma_{1}\left(u_{m}\right)=\left.\frac{\partial u_{m}}{\partial \nu}\right|_{\partial \Omega}, \quad \text { for all } n \in \mathbb{N} .
$$

Replacing those equalities in equation (4.5), we obtain

$$
\begin{aligned}
\int_{\Omega} u_{m}\left(\frac{\partial^{4} v_{m}}{\partial x_{j}^{2} \partial x_{i}^{2}}\right) d x= & -\left[-\int_{\Omega} \frac{\partial^{2} v_{m}}{\partial x_{i}^{2}} \frac{\partial^{2} u_{m}}{\partial x_{i}^{2}} d x+\int_{\partial \Omega} \nu_{i} \gamma_{1}\left(u_{m}\right) \frac{\partial^{2} v_{m}}{\partial x_{j}^{2}} d \sigma\right] \\
& +\int_{\partial \Omega} \gamma_{0}\left(u_{m}\right) \frac{\partial^{3} v_{m}}{\partial x_{i} \partial x_{j}^{2}} \nu_{i} d \sigma
\end{aligned}
$$

The continuity of the trace maps $\gamma_{0}$ and $\gamma_{1}$ allow us to deduce

$$
\lim \gamma_{0}\left(u_{m}\right)=\gamma_{0}(u)=\left.u\right|_{\partial \Omega}, \text { and } \lim \gamma_{1}\left(u_{m}\right)=\gamma_{1}(u)=\left.\frac{\partial u}{\partial \nu}\right|_{\partial \Omega}
$$

Applying the Dominated Convergence Theorem 2.8 to the sequences $u_{m} \rightarrow u, v_{m} \rightarrow v$, $\gamma_{0}\left(u_{m}\right) \rightarrow \gamma_{0}(u), \gamma_{1}\left(u_{m}\right) \rightarrow \gamma_{1}(u), \partial^{k} u_{m} \rightarrow \partial^{k} u$ and $\partial^{k} v_{m} \rightarrow \partial^{k} v$, for all $k=\{1, \ldots, 4\}$, we get from Equation (4.6)

$$
\begin{aligned}
\int_{\Omega} u\left(\frac{\partial^{4} v}{\partial x_{j}^{2} \partial x_{i}^{2}}\right) d x & =-\left[-\int_{\Omega} \frac{\partial^{2} v}{\partial x_{i}^{2}} \frac{\partial^{2} u}{\partial x_{i}^{2}} d x+\int_{\partial \Omega} \gamma_{1}(u) \frac{\partial^{2} v}{\partial x_{j}^{2}} \nu_{i} d \sigma\right] \\
& +\int_{\partial \Omega} \gamma_{0}(u) \frac{\partial^{3} v}{\partial x_{i} \partial x_{j}^{2}} \nu_{i} d \sigma
\end{aligned}
$$

Note that the last two terms on the right side are zero since Theorem 2.37 implies $u \in \operatorname{ker}(\gamma)$, then

$$
\int_{\Omega} u\left(\frac{\partial^{4} v}{\partial x_{i}^{2} \partial x_{j}^{2}}\right) d x=\int_{\Omega} \frac{\partial^{2} u}{\partial x_{i}^{2}} \frac{\partial^{2} v}{\partial x_{j}^{2}} d x
$$

Summing all the terms $i j$, we get

$$
\int_{\Omega} u\left(\Delta^{2} v\right) d x=\int_{\Omega} \Delta u \Delta v d x
$$


We continue with the determination of weak solutions for the SBP system. We already know that $u$ must belong to $H_{0}^{1}(\Omega)$, now we will analyze the space where $\phi$ lies. So, we are going to consider a second subsystem from SBP

$$
\left\{\begin{aligned}
\Delta^{2} \phi-\Delta \phi & =u^{2} \text { in } \Omega \\
u=\Delta \phi=\phi & =0 \text { on } \partial \Omega
\end{aligned}\right.
$$

let $\phi \in C^{4}(\bar{\Omega})$ be a classical solution of (4.10) for all $x \in \bar{\Omega}$. We take $v \in C_{0}^{1}(\Omega) \cap H^{2}(\Omega)$ and multiply the first equation of (4.10) by $v$ and integrate over $\Omega$

$$
\int_{\Omega} \Delta^{2} \phi v-\int_{\Omega} \Delta \phi v=\int_{\Omega} u^{2} v
$$

By Proposition (4.1) and Green's formula (2.10), we have

$$
\int_{\Omega} \Delta \phi \Delta v+\int_{\Omega} \nabla \phi \nabla v=\int_{\Omega} u^{2} v \quad \text { for all } v \in C_{0}^{1}(\Omega) \cap H^{2}(\Omega) .
$$

Observe that this formula also makes sense if $\Delta \phi$ and $\nabla \phi$ are integrable functions on $\Omega$. Also, if $v=\phi$

$$
\int_{\Omega}|\Delta \phi|^{2}+\int_{\Omega}|\nabla \phi|^{2}=\int_{\Omega} u^{2} \phi
$$

That is sufficient that $\phi$ belongs to the intersection of Sobolev spaces $H_{0}^{1}(\Omega)$ and $H^{2}(\Omega)$.

From now on, we denote by

$$
\mathbb{H}_{\Delta^{2}}(\Omega):=H_{0}^{1}(\Omega) \cap H^{2}(\Omega)
$$

and the associated continuous bilinear form to the first equation (4.10) of

$$
b(\phi, v):=\int_{\Omega} \Delta \phi \Delta v+\int_{\Omega} \nabla \phi \nabla v, \text { for all } \phi, v \in \mathbb{H}_{\Delta^{2}}(\Omega) .
$$

\subsubsection{The space $\mathbb{H}_{\Delta^{2}}(\Omega)$.}

Let $\Omega \subset \mathbb{R}^{N}$ be a bounded open set, $N \geq 3$. The Hilbert space

$$
\mathbb{H}_{\Delta^{2}}(\Omega)=H_{0}^{1}(\Omega) \cap H^{2}(\Omega)
$$

When there is no confusion we shall often write $\mathbb{H}_{\Delta^{2}}$ instead of $\mathbb{H}_{\Delta^{2}}(\Omega)$.

Observe that $\mathbb{H}_{\Delta^{2}}(\Omega)$ is a closed subspace of $H^{2}(\Omega)$; it is a Hilbert space with the 
inherited norm from $H^{2}(\Omega)$. Also, as a consequence of Poincaré inequality 2.22 we have

$$
\int_{\Omega}|\Delta \phi|^{2}+|\nabla \phi|^{2}+|\phi|^{2} \leq C\left(\int_{\Omega}|\Delta \phi|^{2}+|\nabla \phi|^{2}\right)
$$

where $C>0$ is depending only on $\Omega$. Thus, we can define a equivalent norm in $\mathbb{H}_{\Delta^{2}}(\Omega)$ given by

$$
\|\phi\|_{\mathbb{H}_{\Delta^{2}}}:=\left(\int_{\Omega}|\Delta \phi|^{2} d x+\int_{\Omega}|\nabla \phi|^{2} d x\right)^{\frac{1}{2}},
$$

which is induced by the inner product

$$
(\phi, v)_{\mathbb{H}_{\Delta^{2}}}:=\int_{\Omega}(\Delta \phi \Delta v+\nabla \phi \nabla v) d x
$$

Observe that it coincides with the continuous bilinear form in (4.11). In fact, $(\cdot, \cdot)_{\mathbb{H}_{\Delta^{2}}}$ satisfies the Cauchy-Schwartz inequality

$$
\left|(\phi, v)_{\mathbb{H}_{\Delta^{2}}}\right| \leq\|\phi\|_{\mathbb{H}_{\Delta^{2}}}\|v\|_{\mathbb{H}_{\Delta^{2}}}
$$

From Inequality $(4.12)$, follows that $(\cdot, \cdot)_{\mathbb{H}_{\Delta^{2}}}$ is a coercive form.

Finally, note that $\mathbb{H}_{\Delta^{2}}(\Omega)$ is a reflexive space since it is a closed subset of a reflexive space.

\subsubsection{Manifolds on Hilbert Spaces}

The space $H_{0}^{1}(\Omega)$ is a linear topological space, $H_{0}^{1}(\Omega)$ together the identity function $i: H_{0}^{1}(\Omega) \rightarrow H_{0}^{1}(\Omega)$ is a $H_{0}^{1}(\Omega)$-structure, according to Definition $2.18 H_{0}^{1}(\Omega)$ is a $H_{0}^{1}(\Omega)$ manifold of class $C^{1}$. By an analogous reasoning, $\mathbb{H}_{\Delta^{2}}(\Omega)$ is a $\mathbb{H}_{\Delta^{2}}(\Omega)$-manifold of class $C^{1}$.

We define the level set on $H_{0}^{1}(\Omega)$

$$
B:=\left\{u \in H_{0}^{1}(\Omega) ; \quad\|u\|_{2}=1\right\}
$$

Proposition 4.2. The level set $B$ is a $H_{0}^{1}(\Omega)$-manifold of class $C^{1}$.

Proof. Set the function

$$
\begin{aligned}
q: H_{0}^{1}(\Omega) & \longrightarrow \mathbb{R}_{\geq 0} \\
u & \longmapsto q(u)=\|u\|_{2} .
\end{aligned}
$$

The Chain Rule Theorem 2.1 and Proposition 2.1 imply that $q \in C^{1}\left(L^{2}(\Omega) \backslash\{0\}\right)$, the derivative $q^{\prime}(u)[u]=\|u\|_{2}$, and $q^{\prime}(u)[u] \neq 0$ for all $u \in B$. Applying Theorem 2.29, we have that the level set $B$ is a $H_{0}^{1}(\Omega)$-manifold. 
We define the set

$$
M:=\left\{(u, \phi) \in H_{0}^{1}(\Omega) \times \mathbb{H}_{\Delta^{2}} ; \quad\|u\|_{2}=1\right\} .
$$

Proposition 4.3. The set $M$ is a $H_{0}^{1}(\Omega) \times \mathbb{H}_{\Delta^{2}}$-manifold of class $C^{1}$.

Proof. Observe that $M$ is the cartesian product of the $H_{0}^{1}(\Omega)$-manifold $B$ and $\mathbb{H}_{\Delta^{2}}$-manifold $\mathbb{H}_{\Delta^{2}}$, both of class $C^{1}$. Thereby, Proposition 2.3 guarantees that $M$ is a $H_{0}^{1}(\Omega) \times \mathbb{H}_{\Delta^{2}}$-manifold of class $C^{1}$.

Let $\left\{u_{1}, \ldots, u_{m}\right\}$ be a set of linearly independent vectors in $H_{0}^{1}(\Omega)$. Define

$$
\mathrm{H}_{m}:=\operatorname{span}\left\{\frac{u_{1}}{\left\|u_{1}\right\|_{2}}, \ldots, \frac{u_{m}}{\left\|u_{m}\right\|_{2}}\right\}
$$

Consider the homeomorphism $t: \mathrm{H}_{m} \rightarrow \mathbb{R}^{m}$ determined by $t\left(\frac{u_{i}}{\left\|u_{i}\right\|_{2}}\right)=e_{i}$, with $i \in\{1, \ldots, m\}$, where $\left\{e_{1}, \ldots, e_{m}\right\}$ is the canonical basis of $\mathbb{R}^{m}$.

Proposition 4.4. There exists an odd homeomorphism between the sphere $\mathbb{S}^{m-1}$ and the set

$$
B \cap \mathrm{H}_{m}=\left\{u \in \mathrm{H}_{m} ; \quad\|u\|_{2}=1\right\} .
$$

Proof. Because of Proposition 2.5, B $\cap \mathrm{H}_{m}$ is homeomorphic to the sphere $\mathbb{S}^{m-1}$.

\subsection{The variational principle for the SBP system}

As we have seen in Chapter 2, the equations in (4.1) determine the motion of a mass-wave particle and every equation of motion obeys a variational principle. Section 4.1 prepared us to propose the variational principle for SBP.

Definition 4.1. A weak solution for the SBP system is a number $\omega \in \mathbb{R}$ and an ordered pair $(u, \phi) \in H_{0}^{1}(\Omega) \times \mathbb{H}_{\Delta^{2}}(\Omega)$ such that the Euler-Lagrange equations hold, i.e.

$$
\int_{\Omega} \nabla u \nabla v d x+\int_{\Omega} \phi u v d x=\int_{\Omega} \omega u v d x, \quad \text { for all } v \in H_{0}^{1}(\Omega)
$$

and

$$
\int_{\Omega} \Delta \phi \Delta v d x+\int_{\Omega} \nabla \phi \nabla v d x=\int_{\Omega} u^{2} v d x, \quad \text { for all } v \in \mathbb{H}_{\Delta^{2}}(\Omega) .
$$

We have seen in Section 3.1 that Euler-Lagrange equations correspond to a single variational principle. We are going to built it. Namely, we have to find a functional whose derivatives are the Euler-Lagrange equations given in Definition 4.1. 
Note that the left side of the Euler-Lagrange equations are a multiple of the bilinear forms $a$ and $b$ defined in (4.3) and (4.11) respectively, i.e.

$$
\begin{gathered}
a(u, v)=\int_{\Omega} \nabla u \nabla v d x+\int_{\Omega} \phi u v d x, \\
b(\phi, v)=\int_{\Omega} \Delta \phi \Delta v d x+\int_{\Omega} \nabla \phi \nabla v d x .
\end{gathered}
$$

Also, the Fréchet derivatives of the continuous bilinear forms, see Proposition 2.1, are given by

$$
a^{\prime}(u, v)[u, v]=2 a(u, v)
$$

and

$$
b^{\prime}(\phi, v)[\phi, v]=2 b(\phi, v)
$$

Since we look for bilinear forms whose derivatives are the forms $a(u, v)$ in $(4.15)$ and $-\frac{1}{2} b(\phi, v)$ in (4.16). Thus, they have the functional

$$
F(u, \phi):=\frac{1}{2} a(u, u)-\frac{1}{4} b(\phi, \phi),
$$

which takes the following form by (4.15) and (4.16)

$$
F(u, \phi)=\frac{1}{2} \int_{\Omega}|\nabla u|^{2} d x+\frac{1}{2} \int_{\Omega} \phi u^{2} d x-\frac{1}{4} \int_{\Omega}|\Delta \phi|^{2} d x-\frac{1}{4} \int_{\Omega}|\nabla \phi|^{2} d x,
$$

defined on the vector space $H_{0}^{1}(\Omega) \times \mathbb{H}_{\Delta^{2}}$ that contains the manifold $M=\left\{(u, \phi) \in H_{0}^{1}(\Omega) \times \mathbb{H}_{\Delta^{2}} ; \quad\|u\|_{2}=1\right\}$.

Thus, the functional $F$ is Fréchet differentiable. Calculating the partial derivative $F_{u}^{\prime}(u, \phi)[v]$ we obtain the expression on the left side of the first Euler-Lagrange equation (4.13), similarly, the partial derivative $F_{\phi}^{\prime}(u, \phi)[v]$ is a multiple of the expression on the left side of the second Euler-Lagrange equation (4.14). Then, finding a weak solution for SBP is equivalent to determining an extreme of $F$ subject to the constraint condition.

Note that

$$
\lim _{t \rightarrow \infty} F(u, t \phi)=-\infty
$$

and

$$
\lim _{t \rightarrow \infty} F(t u, \phi)=\infty .
$$

Therefore, the functional $F$ is unbounded. 


\subsection{The SBP system as a problem of eigenvalues}

The objective of this section is to find the critical points of the functional $F$ on the given manifold $M$. We will use the Lagrange Multipliers Theorem 2.30.

Proposition 4.5. A real number $\omega \in \mathbb{R}$ and a pair $(u, \phi) \in M$ is a weak solution of the $S B P$ system if and only if $(u, \phi)$ is a critical point of the functional $\left.F\right|_{M}$ having $\omega$ as Lagrange multiplier.

Proof. The functional $F$ is differentiable since each of its terms is differentiable, see Definition 2.3. An ordered pair $(u, \phi) \in M$ is a critical point of $\left.F\right|_{M}$ if and only if there exists a Lagrange multiplier $\omega \in \mathbb{R}$ such that

$$
F_{u}^{\prime}(u, \phi)=\omega u
$$

and

$$
F_{\phi}^{\prime}(u, \phi)=0
$$

where $F_{u}^{\prime}(u, \phi)$ and $F_{\phi}^{\prime}(u, \phi)$ denote the partial derivatives of $F$ at $(u, \phi) \in H_{0}^{1}(\Omega) \times \mathbb{H}_{\Delta^{2}}$. Evaluating these derivatives in the direction of any vector-function $v \in \mathbb{H}_{\Delta^{2}}$, we obtain

$$
\begin{gathered}
F_{u}^{\prime}(u, \phi)[v]=\int_{\Omega}(\nabla u \nabla v+u v \phi) d x=\int_{\Omega} \omega u v, \\
F_{\phi}^{\prime}(u, \phi)[v]=-\frac{1}{2} \int_{\Omega}\left(\Delta \phi \Delta v+\nabla \phi \nabla v-u^{2} v\right) d x=0 .
\end{gathered}
$$

Observe that the derivatives (4.20) and (4.21) match respectively to the Euler-Lagrange equations (4.13) and (4.14).

\subsection{Elimination of variables}

Recall from Section 4.2 that the functional in (4.17) is not bounded from below nor from above. Then the usual methods of the Critical Point Theory cannot be directly used. In order to deal with this difficulty, we shall reduce the functional in (4.17) to the study of a functional depending of the single variable $u$, similar to V. Benci and D. Fortunato in [BF98]. After that we will use the Genus Theory to such appropriated functional.

Proposition 4.6. The problem

$$
\Delta^{2} \phi-\Delta \phi=u^{2} \quad \text { in } \Omega
$$

with $u \in H_{0}^{1}(\Omega)$ and the boundary and normalizing conditions

$$
u=\Delta \phi=\phi=0 \quad \text { on } \partial \Omega \quad \text { and } \quad \int_{\Omega} u^{2}=1
$$


has a unique weak solution.

Proof. For every $u \in H_{0}^{1}(\Omega)$, we define the linear functional

$$
\begin{aligned}
f_{u}: \mathbb{H}_{\Delta^{2}}(\Omega) & \longrightarrow \mathbb{R} \\
v & \longmapsto f_{u}[v]=\int_{\Omega} u^{2} v d x
\end{aligned}
$$

The Hölder inequality, Sobolev embedding and $L^{p}$ spaces properties imply

$$
\left|\int_{\Omega} u^{2} v d x\right| \leq\left\|u^{2}\right\|_{\frac{2^{*}}{2}}\|v\|_{\frac{2^{*}}{2 *-2}} \leq C\|u\|_{2^{*}}\|v\|_{\mathbb{H}_{\Delta^{2}}}, \text { with } 2^{*}=\frac{2 N}{N-2}
$$

Then, the functional $f_{u}$ is continuous, and by Theorem 2.6, there exists a unique vector $\phi_{u} \in \mathbb{H}_{\Delta^{2}}(\Omega)$ such that

$$
f_{u}[v]=\left(\phi_{u}, v\right)_{\mathbb{H}_{\Delta^{2}}} \text { for all } v \in \mathbb{H}_{\Delta^{2}}
$$

i. e. $\phi_{u} \in \mathbb{H}_{\Delta^{2}}(\Omega)$ is the unique weak solution of (4.22) satisfying

$$
\int_{\Omega} u^{2} v d x=\int_{\Omega} \Delta \phi_{u} \Delta v d x+\int_{\Omega} \nabla \phi_{u} \nabla v d x
$$

Moreover, the weak solution $\phi_{u}$ depends continuously on $u$.

Set

$$
\Gamma:=\left\{(u, \phi) \in H_{0}^{1}(\Omega) \times \mathbb{H}_{\Delta^{2}} ; \quad F_{\phi}^{\prime}(u, \phi)=0\right\},
$$

where $F_{\phi}^{\prime}$ was calculated in (4.21). Take the manifold $B:=\left\{u \in H_{0}^{1}(\Omega) ;\|u\|_{2}=1\right\}$ and define the map

$$
\begin{aligned}
\Phi: B & \longrightarrow \mathbb{H}_{\Delta^{2}} \\
u & \longmapsto \Phi(u)=\phi_{u} \text { is the unique solution of Equation (4.22). }
\end{aligned}
$$

The Riesz Representation Theorem 2.6 gives us

$$
\Phi(u)=\left(\Delta^{2}-\Delta\right)^{-1} u^{2}
$$

where $\Delta^{2}-\Delta$ is the Riesz isomorphism between $\mathbb{H}_{\Delta^{2}}$ and its dual $\mathbb{H}_{\Delta^{2}}^{\prime}$ defined by

$$
\left\langle\left(\Delta^{2}-\Delta\right) \phi, v\right\rangle=\int_{\Omega} \Delta \phi \Delta v d x+\int_{\Omega} \nabla \phi \nabla v d x, \text { for all } \phi, v \in \mathbb{H}_{\Delta^{2}}
$$

Proposition 4.7. The map $\Phi$ is $C^{1}$ and $\Gamma$ is the graph of $\Phi$.

Proof. Let $u$ be a function in $H_{0}^{1}(\Omega)$; since the Sobolev embedding $2.20, H_{0}^{1}(\Omega) \hookrightarrow L^{2^{*}}(\Omega)$ with $2^{*}=\frac{2 N}{N-2}$ is continuous, the equality $\left\|u^{2}\right\|_{\frac{2^{*}}{2}}=\|u\|_{2^{*}}^{2}<\infty$, that in turn implies that the map $u \mapsto u^{2}$ is $C^{1}$ from $H_{0}^{1}(\Omega)$ into $L^{\frac{2^{*}}{2}}(\Omega)$ which is continuously embedded into dual space 
$\left(\mathbb{H}_{\Delta^{2}}\right)^{\prime}$, and $\left(\Delta^{2}-\Delta\right)^{-1}$ is $C^{1}$. Thus, the map $\Phi$ that was defined by $\Phi(u)=\left(\Delta^{2}-\Delta\right)^{-1} u^{2}$ is $C^{1}$.

Finally, the graph of $\Phi$ is

$$
G r(\Phi):=\left\{(u, \phi) \in M ; \quad\left(\Delta^{2}-\Delta\right)^{-1} u^{2}=\phi\right\}
$$

And note that $(u, \phi) \in G r(\Phi)$ means that $\left(\Delta^{2}-\Delta\right) \phi=u^{2}$ which is equivalent to $F_{\phi}^{\prime}(u, \phi)=0$ which in turn is also equivalent to $(u, \phi) \in \Gamma$.

For a given $u \in B, \phi_{u}$ solves the second equation of SBP and

$$
\left(\Delta^{2}-\Delta\right) \phi_{u}=u^{2}
$$

Evaluating $\left(\left(\Delta^{2}-\Delta\right) \phi_{u}\right)\left[\phi_{u}\right]$, we have

$$
\frac{1}{2} \int_{\Omega}\left|\Delta \phi_{u}\right|^{2} d x+\frac{1}{2} \int_{\Omega}\left|\nabla \phi_{u}\right|^{2} d x=\frac{1}{2} \int_{\Omega} u^{2} \phi_{u} d x
$$

hence

$$
\frac{1}{4} \int_{\Omega}\left|\Delta \phi_{u}\right|^{2} d x+\frac{1}{2} \int_{\Omega}\left|\nabla \phi_{u}\right|^{2} d x=\frac{1}{2} \int_{\Omega} u^{2} \phi_{u} d x-\frac{1}{4} \int_{\Omega}\left|\Delta \phi_{u}\right|^{2} d x .
$$

Replacing $\Phi(u)=\phi_{u}$ in the functional $\left.F\right|_{M}$ in (4.17) we define the functional $J$ as follows

$$
\begin{aligned}
J(u) & :=F(u, \Phi(u)) \\
& =\frac{1}{2} \int_{\Omega}|\nabla u|^{2} d x+\frac{1}{2} \int_{\Omega} u^{2} \phi_{u} d x-\frac{1}{4} \int_{\Omega}\left|\Delta \phi_{u}\right|^{2} d x-\frac{1}{4} \int_{\Omega}\left|\nabla \phi_{u}\right|^{2} d x .
\end{aligned}
$$

Using (4.28), the functional $J$ takes the form of a positive functional

$$
\begin{aligned}
J(u) & =\frac{1}{2} \int_{\Omega}|\nabla u|^{2} d x+\frac{1}{4} \int_{\Omega}\left|\Delta \phi_{u}\right|^{2} d x+\frac{1}{2} \int_{\Omega}\left|\nabla \phi_{u}\right|^{2} d x-\frac{1}{4} \int_{\Omega}\left|\nabla \phi_{u}\right|^{2} d x \\
& =\frac{1}{2} \int_{\Omega}|\nabla u|^{2} d x+\frac{1}{4} \int_{\Omega}\left|\Delta \phi_{u}\right|^{2} d x+\frac{1}{4} \int_{\Omega}\left|\nabla \phi_{u}\right|^{2} d x .
\end{aligned}
$$

Note that multiplying the bilinear form $b$ in (4.16) by the constant $-\frac{1}{2}$ in Equation (4.17) to obtain the functional $F$ was necessary, otherwise the terms depending on the function $\phi_{u}$ would have disappeared from the reduced functional $J$.

As a consequence, the functional $\left.J\right|_{B}$ is bounded from below and even. Moreover, by Proposition 4.7 it is $C^{1}$. Then, the Fréchet derivative of functional $\left.J\right|_{B}$ at $u$ in the direction of $u$ is the total derivative of $\left.F\right|_{M}$, see Theorem 2.2 and the Chain Rule Theorem 2.1, and it is given by

$$
\left.J\right|_{B} ^{\prime}(u)[u]=F_{u}^{\prime}\left(u, \phi_{u}\right)[u]+F_{\phi}^{\prime}\left(u, \phi_{u}\right)[u] \phi_{u}^{\prime} .
$$


Equation (4.19) in Proposition 4.5 implies

$$
F_{\phi}^{\prime}\left(u, \phi_{u}\right) \phi_{u}^{\prime}=0
$$

and equations (4.20) and (4.18) imply

$$
F_{u}^{\prime}\left(u, \phi_{u}\right)[u]=\int_{\Omega}|\nabla u|^{2} d x+\int_{\Omega} u^{2} \phi_{u} d x=\omega \int_{\Omega} u^{2} d x
$$

From these equalities and the normalization condition $\|u\|_{2}=1$, the derivative of the functional $\left.J\right|_{B}$ in $(4.31)$ is

$$
\left.J\right|_{B} ^{\prime}(u)[u]=\int_{\Omega}|\nabla u|^{2} d x+\int_{\Omega} u^{2} \phi_{u} d x=\omega
$$

The following proposition describes the relation between critical points of the $\left.F\right|_{M}$ and critical points of $\left.J\right|_{B}$.

Proposition 4.8. Let $(u, \phi) \in M$ and $\omega \in \mathbb{R}$. The following statements are equivalent

1. The pair $(u, \phi)$ is a critical point of $\left.F\right|_{M}$ having $\omega$ as Lagrange multiplier.

2. The function $u$ is a critical point of $\left.J\right|_{B}$ having $\omega$ as Lagrange multiplier and $\phi=\Phi(u)$.

Proof. Assuming (1) by Lagrange Multiplier Theorem 2.30, means that

$$
F_{u}^{\prime}(u, \phi)+F_{\phi}^{\prime}(u, \phi) \phi^{\prime}=\omega u
$$

Now, by Proposition 4.5 we have

$$
\left.J\right|_{B} ^{\prime}(u)=F_{u}^{\prime}(u, \phi)+F_{\phi}^{\prime}(u, \phi) \phi^{\prime}=\omega u
$$

Again, by Lagrange Multiplier Theorem 2.30, it means that $u$ is a critical point of $\left.J\right|_{B}$ as in the Claim (2).

Conversely, assuming (2), we have $(u, \Phi(u)) \in G r(\Phi)$ which implies $F_{\phi}^{\prime}(u, \Phi(u))=0$ (see proof of Proposition 4.7). Then, the derivative

$$
\left.J\right|_{B} ^{\prime}(u)=F_{u}^{\prime}(u, \Phi(u))+F_{\phi}^{\prime}(u, \Phi(u)) \Phi^{\prime}(u)=F_{u}^{\prime}(u, \Phi(u)) .
$$

Since $\omega$ is the Lagrange multiplier of $\left.J\right|_{B} ^{\prime}(u)$, we have $F_{u}^{\prime}(u, \Phi(u))=\omega u$, thereby we conclude (1). 


\subsection{Properties of the functional $J$}

We recall that the main purpose of this section is to determine the extremal points in $M$ of the functional $F$. In the last section, we saw that $\left.J\right|_{B}$ in (4.30) is a positive functional unlike the unbounded functional $\left.F\right|_{M}$. Therefore, we can apply the Critical Point Theory to the functional $\left.J\right|_{B}$. The lemmas of this section will be used to prove the main theorem in the next section.

Lemma 4.1 (Palais-Smale Condition). The functional $\left.J\right|_{B}$ satisfies the Palais-Smale condition, i.e. any $(P S)$-sequence for the functional $\left.J\right|_{B}$ contains a convergent subsequence in $B \subset H_{0}^{1}(\Omega)$.

Proof. Let $\left(u_{n}\right) \subset B$ be a $(P S)$-sequence for $\left.J\right|_{B}$. By Definition 2.24, the sequence $\left(J\left(u_{n}\right)\right)$ is bounded and there exist two sequences $\left(\lambda_{n}\right)$ in $\mathbb{R}$ and $\left(v_{n}\right)$ in $H^{-1}(\Omega)$, where $H^{-1}(\Omega)$ is the dual space of $H_{0}^{1}(\Omega)$, such that $v_{n} \rightarrow 0$ in $H^{1}(\Omega)$ and

$$
\left.J\right|_{B} ^{\prime}\left(u_{n}\right)=\lambda_{n} u_{n}+v_{n}
$$

Which by the derivative of $\left.J\right|_{B} ^{\prime}\left(u_{n}\right)$ (4.31) and the definition of map $\Phi$ in (4.26) the last equality takes the form

$$
F_{u}^{\prime}\left(u_{n}, \Phi\left(u_{n}\right)\right)+F_{\phi}^{\prime}\left(u_{n}, \Phi\left(u_{n}\right)\right) \Phi^{\prime}\left(u_{n}\right)=\lambda_{n} u_{n}+v_{n}
$$

By Proposition 4.7 we obtained that $\left(u_{n}, \Phi\left(u_{n}\right)\right) \in \Gamma$, then (4.35) becomes

$$
F_{u}^{\prime}\left(u_{n}, \Phi\left(u_{n}\right)\right)=\lambda_{n} u_{n}+v_{n}
$$

By assumption the sequence $\left(J\left(u_{n}\right)\right)$ is bounded, where $\left.J\right|_{B}$ is given by $(4.30)$, then

$$
\left(\frac{1}{2} \int_{\Omega}\left|\nabla u_{n}\right|^{2} d x+\frac{1}{4} \int_{\Omega}\left|\Delta \Phi\left(u_{n}\right)\right|^{2} d x+\frac{1}{4} \int_{\Omega}\left|\nabla \Phi\left(u_{n}\right)\right|^{2} d x\right) \text { is bounded. }
$$

By the Poincaré Inequality (2.22) and Claim (4.37) the sequence

$$
\left(u_{n}\right) \text { is bounded in } H_{0}^{1}(\Omega) \text {. }
$$

Also, thanks to Claim (4.37) the sequence

$$
\left(\phi_{u_{n}}\right) \text { is bounded in } \mathbb{H}_{\Delta^{2}}(\Omega) \text {. }
$$

Furthermore, $\left\|u_{n}\right\|_{2}=1$ for all $n \in \mathbb{N}$ and $\Phi$ is an injection of class $C^{1}$ by Proposition 4.7, 
then there exists $\epsilon>0$ such that

$$
\left\|\phi_{u_{n}}\right\|_{\mathbb{H}_{\Delta^{2}}}=\left\|\Phi\left(u_{n}\right)\right\|_{\mathbb{H}_{\Delta^{2}}} \leq \epsilon\left\|u_{n}\right\|_{2}=\epsilon
$$

Also, we claim that the sequence of real numbers

$$
\left(\lambda_{n}\right) \text { is bounded. }
$$

Indeed, we recall that a derivative is a operator, applying the partial derivative in Equality (4.36) at $u_{n}$, we get

$$
F_{u}^{\prime}\left(u_{n}, \Phi\left(u_{n}\right)\right)\left[u_{n}\right]=\int_{\Omega}\left|\nabla u_{n}\right|^{2} d x+\int_{\Omega} u_{n}^{2} \Phi\left(u_{n}\right) d x=\lambda_{n}+\left\langle v_{n}, u_{n}\right\rangle .
$$

We will analyze the terms of the last equation.

First, Claim (4.37) implies that $\int_{\Omega}\left|\nabla u_{n}\right|^{2} d x$ is bounded.

Second, by Hölder inequality and the Sobolev embeddings $H_{0}^{1}(\Omega) \hookrightarrow L^{2}(\Omega)$ and $H_{0}^{1}(\Omega) \hookrightarrow L^{4}(\Omega)$, we obtain

$$
\begin{aligned}
\left|\int_{\Omega} u_{n}^{2} \Phi\left(u_{n}\right) d x\right| & \leq\left(\int_{\Omega}\left|u_{n}^{2}\right|^{2}\right)^{\frac{1}{2}}\left(\int_{\Omega}\left|\Phi\left(u_{n}\right)\right|^{2}\right)^{\frac{1}{2}} \\
& =\left(\int_{\Omega}\left|u_{n}\right|^{4}\right)^{\frac{1}{4} \cdot{ }^{2}}\left(\int_{\Omega}\left|\Phi\left(u_{n}\right)\right|^{2}\right)^{\frac{1}{2}} \\
& \leq C_{1}\left\|u_{n}\right\|_{4}^{2}\left\|\Phi\left(u_{n}\right)\right\|_{\mathbb{H}_{\Delta^{2}}} \\
& \leq C_{2}\left\|u_{n}\right\|_{H_{0}^{1}}^{2}\left\|\Phi\left(u_{n}\right)\right\|_{\mathbb{H}_{\Delta^{2}}},
\end{aligned}
$$

which implies that $\left|\int_{\Omega} u_{n}^{2} \Phi\left(u_{n}\right) d x\right|$ is bounded because of (4.38) and (4.40).

Third, $\left\langle v_{n}, u_{n}\right\rangle \rightarrow 0$ in $\mathbb{R}$, since $v_{n} \rightarrow 0$ in $H^{-1}(\Omega)$ and $\left\|u_{n}\right\|_{2}=1$.

Therefore, the sequence $\left(\lambda_{n}\right)$ is bounded.

Now, (4.36) is the Euler-Lagrange Equation associated to

$$
-\Delta u_{n}+u_{n} \phi_{u_{n}}-\lambda_{n} u_{n}=v_{n}
$$

Applying the inverse $\Delta^{-1}: H^{-1}(\Omega) \rightarrow H_{0}^{1}(\Omega)$ of the Riesz isomorphism $\Delta$, we obtain

$$
-u_{n}+\Delta^{-1}\left(u_{n} \phi_{u_{n}}\right)-\lambda_{n} \Delta^{-1} u_{n}=\epsilon_{n}
$$

where $\epsilon_{n}:=\Delta^{-1} v_{n}$. Note that $\epsilon_{n} \rightarrow 0$ in $H_{0}^{1}(\Omega)$, since $v_{n} \rightarrow 0$ in $H^{-1}(\Omega)$ and $\Delta^{-1}$ is continuous. We will analyze each term of this equation in order to show the existence of a strongly convergent subsequence of $u_{n}$ in $H_{0}^{1}(\Omega)$.

First, considering the sequences $\left(u_{n}\right)$ and $\left(\phi_{u_{n}}\right)$ we define $L:=\left\{u_{n} ; n \in \mathbb{Z}_{+}\right\} \subset H_{0}^{1}(\Omega)$ 
and $P:=\left\{\phi_{u_{n}} ; n \in \mathbb{Z}_{+}\right\} \subset \mathbb{H}_{\Delta^{2}}$ which are bounded by (4.38) and (4.39). Since the Hilbert spaces $H_{0}^{1}(\Omega)$ and $\mathbb{H}_{\Delta^{2}}$ are reflexive spaces by Banach-Alaoglu Theorem 2.5, the sets $L$ and $P$ are relatively compacts. This means that there exist $\left(u, \phi_{u}\right) \in H_{0}^{1}(\Omega) \times \mathbb{H}_{\Delta^{2}}$ and weakly convergent subsequences $\left(u_{n_{i}}\right)_{i}$ and $\left(\phi_{u_{n_{i}}}\right)_{i}$ such that

$$
u_{n_{i}} \rightarrow u \text { in } H_{0}^{1}(\Omega) \text { and } \phi_{u_{n_{i}}} \rightarrow \phi_{u} \text { in } \mathbb{H}_{\Delta^{2}}
$$

By the Sobolev embedding 2.20, $H_{0}^{1}(\Omega)$ is compactly embedded into $L^{p}(\Omega)$ for $p<2^{*}$, with $2^{*}=\frac{2 N}{N-2}$, then

$$
u_{n_{i}} \rightarrow u \text { strongly in } L^{p}(\Omega), p<2^{*} .
$$

In particular, $u_{n_{i}} \rightarrow u$ strongly in $L^{2}(\Omega)$, then by Theorem 2.24 we have

$$
u_{n_{i}} \rightarrow u \text { strongly in } H^{-1}
$$

Also, the Sobolev embedding 2.20 holds that $\mathbb{H}_{\Delta^{2}}(\Omega)$ is compactly embedded into $L^{p}(\Omega)$ for $p<2^{*}$, thereby

$$
\phi_{u_{n_{i}}} \rightarrow \phi_{u} \text { strongly in } L^{p}(\Omega), p<2^{*} .
$$

Second, from claims (4.47) and (4.48) we obtain that

$$
\left(u_{n_{i}} \phi_{u_{n_{i}}}\right)_{i} \text { is bounded in } H^{-1} \text {. }
$$

Third, the continuity of the isomorphism $\Delta^{-1}$ and the results in (4.41), (4.47) and $\epsilon_{n} \rightarrow 0$ in $H_{0}^{1}(\Omega)$ imply that there exists a subsequence $\left(u_{n_{i_{k}}}\right)_{k}$ such that

$$
\alpha_{n_{i_{k}}}:=\lambda_{n_{i_{k}}} \Delta^{-1} u_{n_{i_{k}}}+\epsilon_{n_{i_{k}}} \text { converges strongly in } H_{0}^{1}(\Omega) \text {. }
$$

Then, Equation (4.44) takes the form

$$
-u_{n_{i_{k}}}+\Delta^{-1}\left(u_{n_{i_{k}}} \phi_{u_{n_{i_{k}}}}\right)=\alpha_{n_{i_{k}}}
$$

Fourth, in order to prove that $u_{n_{i_{k}}}$ converges strongly in $H_{0}^{1}(\Omega)$, it remains to show that

$$
u_{n_{i_{k}}} \phi_{u_{n_{i_{k}}}} \rightarrow u \phi_{u} \text { strongly in } H^{-1}(\Omega) .
$$

Let $2 \leq p<2^{*}$ and consider its conjugate $\frac{2^{*}}{2^{*}-1}<q=\frac{p}{p-1} \leq 2$. By the Triangle inequality

$$
\left\|u_{n_{i_{k}}} \phi_{u_{n_{i_{k}}}}-u \phi_{u}\right\|_{q} \leq\left\|u_{n_{i_{k}}} \phi_{u_{n_{i_{k}}}}-u_{n_{i_{k}}} \phi_{u}\right\|_{q}+\left\|u_{n_{i_{k}}} \phi_{u}-u \phi_{u}\right\|_{q}
$$

Applying the extension of Hölder's inequality (2.12) with the identity $\frac{1}{q}=\frac{1}{2^{*}}+\frac{2^{*}-q}{2^{*} q}$, we 
obtain

$$
\left\|u_{n_{i_{k}}} \phi_{u_{n_{i_{k}}}}-u \phi_{u}\right\|_{q} \leq\left\|u_{n_{i_{k}}}\right\|_{2^{*}}\left\|\phi_{u_{n_{i_{k}}}}-\phi_{u}\right\|_{\frac{2^{*} q}{2^{*}-q}}+\left\|u_{n_{i_{k}}}-u\right\|_{\frac{2^{*} q}{2^{*}-q}}\left\|\phi_{u}\right\|_{2^{*}} .
$$

Observe that the subsequence $\left(u_{n_{i_{k}}}\right)_{k}$ is bounded in $L^{2^{*}}(\Omega)$ since $u_{n_{i}} \rightarrow u$ in $H_{0}^{1}(\Omega)$ and the compact embedding $H_{0}^{1} \hookrightarrow L^{2^{*}}$. Noting that $\frac{2^{*} q}{2^{*}-q} \leq 2^{*}$, by Claim (4.48) we conclude that

$$
u_{n_{i_{k}}} \rightarrow u \text { and } \quad \phi_{u_{n_{i_{k}}}} \rightarrow \phi_{u} \text { in } L^{\frac{2^{*} q}{2^{*}-q}}(\Omega)
$$

so

$$
\left\|u_{n_{i_{k}}}-u\right\|_{\frac{2^{*} q}{2^{*}-q}} \rightarrow 0 \text { and }\left\|\phi_{u_{n_{i_{k}}}}-\phi_{u}\right\|_{\frac{2^{*} q}{2^{*}-q}} \rightarrow 0
$$

Claim 4.39 and the embedding $\mathbb{H}_{\Delta^{2}} \hookrightarrow L^{2^{*}}$ guarantee that $\phi_{u} \in \mathbb{H}_{\Delta^{2}}$ has finite norm in $L^{2^{*}}(\Omega)$. Therefore, by Inequation (4.54) we get

$$
\left\|u_{n_{i_{k}}} \phi_{u_{n_{i_{k}}}}-u \phi_{u}\right\|_{q} \rightarrow 0
$$

Finally, the embedding $L^{q} \hookrightarrow H^{-1}$ implies Claim (4.52).

Now, we present some deformation lemmas in the context of Genus Theory. We recall that given a closed and symmetric subset $A$ of a Banach space, the set $A$ has genus $n$, denoted by $\gamma(A)=n$, if there exists and odd map $h \in C\left(A, \mathbb{R}^{n}\right)$ and $n$ is the smallest integer having this property. If $A=\emptyset$, we say that $\gamma(A)=0$; and if there is not any integer satisfying the property, we set $\gamma(A)=\infty$. We presented more properties of Genus Theory in Section 2.6.

Lemma 4.2. For any integer $m$ there exists a compact symmetric subset $\mathrm{K}$ of $B=\left\{u \in H_{0}^{1}(\Omega) ;\|u\|_{2}=1\right\}$ such that $\gamma(\mathrm{K})=m$.

Proof. Let $\mathrm{H}_{m}:=\operatorname{span}\left\{u_{1}, \ldots, u_{m}\right\}$ be a $m$-dimensional subspace of $H_{0}^{1}(\Omega)$. Define

$$
\mathrm{K}:=B \cap \mathrm{H}_{m}=\left\{u \in \mathrm{H}_{m} ;\|u\|_{2}=1\right\}
$$

By Proposition 4.4 there exists an odd homeomorphism between $\mathrm{K}$ and $\mathbb{S}^{m-1}$. Then, by Theorem 2.27

$$
\gamma(\mathrm{K})=\gamma\left(\mathbb{S}^{m-1}\right)=m
$$

Lemma 4.3. For any $b \in \mathbb{R}$ the sublevel

$$
J^{b}=\{u \in B ; \quad J(u) \leq b\}
$$


has finite genus.

Proof. Suppose by contradiction that there exists a real number $b$ such that $\gamma\left(J^{b}\right)=\infty$, this means that the set

$$
D:=\left\{b \in \mathbb{R} ; \quad \gamma\left(J^{b}\right)=\infty\right\} \neq \emptyset .
$$

We know that $\left.J\right|_{B}$ is bounded from below, then

$$
-\infty<\underline{b}:=\inf D<\infty .
$$

We claim that $\underline{b} \notin D$. Indeed, since $\left.J\right|_{B}$ satisfies the Palais-Smale condition (Lemma 4.1), the set

$$
K_{\underline{b}}:=\left\{u \in B ; \quad J(u)=\underline{b},\left.J\right|_{B} ^{\prime}(u)=0\right\}
$$

is compact. By the fifth claim of Proposition 2.2, there exists a closed symmetric neighborhood $U_{\underline{b}}$ of $K_{\underline{b}}$ such that $\gamma\left(U_{\underline{b}}\right)<\infty$, then $\underline{b} \notin D$.

Although, $J$ is an even functional such that satisfies the $(P S)$-condition and $U_{\underline{b}}$ is a open neighborhood of critical points at the level $\underline{b}$. By Theorem 2.32 there exist $\epsilon>0$ and an odd homeomorphism $\eta$ such that $\eta\left(1, J^{\underline{b}+\epsilon} \backslash U_{\underline{b}}\right) \subset J^{\underline{b}-\epsilon}$. Using properties (2), (4) and (6) of Proposition 2.2, we get

$$
\gamma\left(J^{\underline{b}+\epsilon}\right) \leq \gamma\left(J^{\underline{b}+\epsilon} \backslash U_{\underline{b}}\right)+\gamma\left(U_{\underline{b}}\right) \leq \gamma\left(J^{\underline{b}-\epsilon}\right)+\gamma\left(U_{\underline{b}}\right)<\infty
$$

which goes against the fact that $\underline{b}$ is inf $D$. Therefore for all $b \in \mathbb{R}$ the genus $\gamma\left(J^{b}\right)<\infty$.

\subsection{Main Result}

This section is devoted to prove Theorem 4.3, the main result of this work. It guarantees the multiplicity of solutions of the SBP system. Theorem 4.3 is a direct consequence of Theorem 4.2, which shows the existence of infinite critical points of the restricted functional $\left.F\right|_{M}$. Due to the relationship between critical points of $\left.F\right|_{M}$ and critical points of $\left.J\right|_{B}$ described in Section 4.4, Theorem 4.2 is a consequence of the next result.

Theorem 4.1. There exists a sequence $\left(u_{n}\right)$ of critical points of $\left.J\right|_{B}$ having Lagrange multipliers $\omega_{n} \rightarrow \infty$. Furthermore, $\left\|u_{n}\right\|_{H_{0}^{1}(\Omega)} \rightarrow \infty$.

Proof. Let $n$ be a positive integer. By Lemma 4.3, there exists a positive integer $k=k(n)$ such that

$$
\gamma\left(J^{n}\right)=k
$$


Now, consider the collection

$$
\mathcal{A}_{k+1}:=\{A \subset B ; \quad A \text { is symmetric and closed with } \gamma(A)=k+1\} .
$$

By Lemma 4.2, there exists a compact set $\mathrm{K} \subset B$ such that $\mathrm{K} \in \mathcal{A}_{k+1}$, then $\mathcal{A}_{k+1} \neq \emptyset$.

We have that

$$
\gamma(A)>\gamma\left(J^{n}\right), \text { for all } A \in \mathcal{A}_{k+1},
$$

then by monotonicity property of genus (second claim of Proposition 2.2 ) $A \not \subset J^{n}$, thus

$$
\sup J(A)>n, \text { for all } A \in \mathcal{A}_{k+1},
$$

and consequently

$$
b_{n}:=\inf \left\{\sup J(A) ; A \in \mathcal{A}_{k+1}\right\} \geq n
$$

The functional $J$ is even and $\left.J\right|_{B}$ satisfies the $P S$-condition, by Lemma 4.1, then follows from Theorem 2.33 that

$$
b_{n} \text { is a critical value of }\left.J\right|_{B} \text {. }
$$

By the Lagrange Multipliers Theorem 2.30, for any $n$ there exist $\omega_{n} \in \mathbb{R}$ and $u_{n} \in B$ such that

$$
J^{\prime}\left(u_{n}\right)=\omega_{n} u_{n} \quad \text { and } \quad J\left(u_{n}\right)=b_{n} \geq n .
$$

We claim that $\omega_{n} \rightarrow \infty$ as $n \rightarrow \infty$. Indeed, $J^{\prime}\left(u_{n}\right)=\omega_{n} u_{n}$ and Proposition 4.8 imply

$$
F_{u}^{\prime}\left(u_{n}, \phi_{u_{n}}\right)=\omega_{n} u_{n}, \quad \text { where } \phi_{u_{n}}=\Phi\left(u_{n}\right) .
$$

Evaluating the partial derivative at $u_{n}$ we get $F_{u}^{\prime}\left(u_{n}, \phi_{u_{n}}\right)\left[u_{n}\right]=\omega_{n} u_{n}\left[u_{n}\right]$. Equation (4.32) and $\left\|u_{n}\right\|_{2}=1$ imply

$$
\frac{1}{2} \int_{\Omega}\left|\nabla u_{n}\right|^{2} d x+\frac{1}{2} \int_{\Omega} \phi_{u_{n}} u_{n}^{2} d x=\frac{1}{2} \omega_{n} .
$$

Replacing the above equation in the functional $J$ in (4.29), we have

$$
J\left(u_{n}\right)=\frac{1}{2} \omega_{n}-\frac{1}{4} \int_{\Omega}\left|\Delta \phi_{u_{n}}\right|^{2} d x-\frac{1}{4} \int_{\Omega}\left|\nabla \phi_{u_{n}}\right|^{2} d x
$$

Since $J\left(u_{n}\right)=b_{n}$, the equation above becomes

$$
\omega_{n}=2 b_{n}+\frac{1}{2} \int_{\Omega}\left|\Delta \phi_{u_{n}}\right|^{2} d x+\frac{1}{2} \int_{\Omega}\left|\nabla \phi_{u_{n}}\right|^{2} d x
$$

which shows that $\omega_{n} \rightarrow \infty$ as $n \rightarrow \infty$ because $b_{n} \geq n$. 
Recalling Equation (4.27), we rewrite Equation (4.59) as

$$
\omega_{n}=\int_{\Omega}\left|\nabla u_{n}\right|^{2} d x+\int_{\Omega}\left|\Delta \phi_{u_{n}}\right|^{2} d x+\int_{\Omega}\left|\nabla \phi_{u_{n}}\right|^{2} d x
$$

where $\phi_{u_{n}}=\Phi\left(u_{n}\right)$ and $u_{n} \in H_{0}^{1}(\Omega)$. From equation above and the continuity of $\Phi$, we have that

$$
\omega_{n} \leq\left\|u_{n}\right\|_{H_{0}^{1}(\Omega)}^{2}+\left\|\Phi\left(u_{n}\right)\right\|_{\mathbb{H}_{\Delta^{2}}}^{2} \leq C\left\|u_{n}\right\|_{H_{0}^{1}(\Omega)}^{2} .
$$

Therefore, since $\omega_{n} \rightarrow \infty$ as $n \rightarrow \infty$, then

$$
\left\|u_{n}\right\|_{H_{0}^{1}(\Omega)} \rightarrow \infty \text { as } n \rightarrow \infty
$$

Theorem 4.2. Let $\Omega$ be an open bounded set of $\mathbb{R}^{N}, N \geq 3$. Then there is a sequence $\left(u_{n}, \phi_{n}\right) \in M$ of critical points of $\left.F\right|_{M}$ whose Lagrange multipliers $\omega_{n} \rightarrow \infty$. Furthermore, $\left\|u_{n}\right\|_{H_{0}^{1}(\Omega)} \rightarrow \infty$.

Proof. Theorem 4.1 provides us a sequence $\left(u_{n}\right)$ of critical points of $\left.J\right|_{B}$ such that the Lagrange multipliers $\omega_{n} \rightarrow \infty$ and $\left\|u_{n}\right\|_{H_{0}^{1}(\Omega)} \rightarrow \infty$. Setting $\phi_{n}:=\Phi\left(u_{n}\right)$, by Proposition 4.8 we have that $\left(u_{n}, \phi_{n}\right)$ is the sequence of critical points of $\left.F\right|_{M}$ required.

Theorem 4.3. Let $\Omega$ be an open bounded set of $\mathbb{R}^{N}, N \geq 3$. There is a sequence $\left(\omega_{n}, u_{n}, \phi_{n}\right)$ such that $\left(\omega_{n}\right) \subset \mathbb{R}, \omega_{n} \rightarrow \infty$ and $u_{n}, \phi_{n}$ real functions solving the SBP system. Furthermore, $\left\|u_{n}\right\|_{H_{0}^{1}(\Omega)} \rightarrow \infty$.

Proof. Theorem 4.2 provides us a sequence $\left(u_{n}, \phi_{n}\right)$ of critical points of $\left.F\right|_{M}$ such that the Lagrange multipliers $\omega_{n} \rightarrow \infty$ and $\left\|u_{n}\right\|_{H_{0}^{1}(\Omega)} \rightarrow \infty$. By Proposition 4.5, the triples of the sequence $\left(\omega_{n}, u_{n}, \phi_{n}\right) \in \mathbb{R} \times M$ are solutions of the SBP system in (4.1). 


\section{Chapter 5}

\section{The SBP system with a parameter}

In this chapter, we study the following Schrödinger-Bopp-Podolsky system

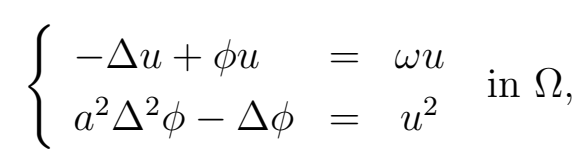

with the boundary conditions

$$
u=\Delta \phi=\phi=0 \quad \text { on } \quad \partial \Omega
$$

and normalizing condition

$$
\int_{\Omega} u^{2}=1
$$

where $\Omega$ is a bounded open subset of $\mathbb{R}^{N}, N \geq 3, a$ and $\omega$ are positive constants. The system 5.1 , together with the boundary and normalizing conditions, will be called SBP-a system. We show results about existence of solutions and their regularity of the solutions. Also, we will see that these solutions tend to the solutions of the classical Schrödinger-Poisson system as $a \rightarrow 0$. For this, we consider P. d'Avenia and G. Siciliano [dS19].

\subsection{Solution of the SBP- $a$ system}

Fixing $a>0$, we aim to show the existence of real numbers $\omega$ and real functions $u$ and $\phi$ satisfying the SBP- $a$ system. Note that we get the SBP system when $a=1$. Thereby, making certain settings in the solution spaces we obtain similar results to those we obtained in Chapter 4 for the SBP system.

We follow an analogous procedure as in Section 3.1 to define the weak solution of the SBP- $a$ system. We consider classical solutions of SBP- $a u$ and $\phi$, then we multiply every 
equation of the system by $v \in C_{0}^{1}(\Omega)$. Integrating over $\Omega$, we obtain

$$
\begin{aligned}
-\int_{\Omega}(\Delta u) v+\int_{\Omega} \phi u v & =\omega \int_{\Omega} u v, \\
a^{2} \int_{\Omega} \Delta^{2} \phi v-\int_{\Omega} \Delta \phi v & =\int_{\Omega} u^{2} v .
\end{aligned}
$$

Observe that the equalities above are satisfied if $u$ belongs to $H_{0}^{1}(\Omega)$ and $\phi$ belongs to $\mathbb{H}_{\Delta^{2}}(\Omega)$ with the norm induced by the inner product defined as:

$$
(\phi, \psi)_{a}=\int_{\Omega} \nabla \phi \nabla \psi d x+a^{2} \int_{\Omega} \Delta \phi \Delta \psi d x, \quad \text { for all } \phi, \psi \in \mathbb{H}_{\Delta^{2}}(\Omega)
$$

We use the generalized Green's Formulas in the above equations (5.2) and (5.3) to obtain the corresponding Euler-Lagrange equations associated to SBP- $a$.

We will often use the following result.

Proposition 5.1. Let a be a positive real number. In the space $\mathbb{H}_{\Delta^{2}}(\Omega)$, the following norms are equivalent

$$
\|u\|_{a}=\left(a^{2} \int_{\Omega}|\Delta u|^{2} d x+\int_{\Omega}|\nabla u|^{2} d x\right)^{\frac{1}{2}}
$$

and

$$
\|u\|_{\mathbb{H}_{\Delta^{2}}(\Omega)}=\left(\int_{\Omega}|\Delta u|^{2} d x+\int_{\Omega}|\nabla u|^{2} d x\right)^{\frac{1}{2}} .
$$

Proof. Indeed, we estimate

$$
a^{2}\left[\int_{\Omega}|\Delta \phi|^{2}+\int_{\Omega}|\nabla \phi|^{2}\right] \leq a^{2} \int_{\Omega}|\Delta \phi|^{2}+\int_{\Omega}|\nabla \phi|^{2} \leq \int_{\Omega}|\Delta \phi|^{2}+\int_{\Omega}|\nabla \phi|^{2}, \quad \text { if } 0<a<1
$$

and

$$
\int_{\Omega}|\Delta \phi|^{2}+\int_{\Omega}|\nabla \phi|^{2} \leq a^{2} \int_{\Omega}|\Delta \phi|^{2}+\int_{\Omega}|\nabla \phi|^{2} \leq a^{2}\left[\int_{\Omega}|\Delta \phi|^{2}+\int_{\Omega}|\nabla \phi|^{2}\right], \quad \text { if } a \geq 1 .
$$

Definition 5.1. Let $a>0$. A weak solution for the system $S B P-a$ is a number $\omega^{a} \in \mathbb{R}$ and an ordered pair $\left(u^{a}, \phi^{a}\right) \in H_{0}^{1}(\Omega) \times \mathbb{H}_{\Delta^{2}}(\Omega)$ such that the Euler-Lagrange equations holds, i.e.

$$
\int_{\Omega} \nabla u \nabla v+\int_{\Omega} \phi u v=\omega \int_{\Omega} u v \quad \text { for all } v \in H_{0}^{1}(\Omega)
$$

and

$$
a^{2} \int_{\Omega} \Delta \phi \Delta v+\int_{\Omega} \nabla \phi \nabla v=\int_{\Omega} u^{2} v \quad \text { for all } v \in \mathbb{H}_{\Delta^{2}}(\Omega)
$$


In order to lighten the notation, while working with a fixed $a$, we write $\omega^{a}, u^{a}$, and $\phi^{a}$ simply as $\omega, u$, and $\phi$. Later, we will denote the weak solution $\omega^{a}, u^{a}, \phi^{a}$ as the triple $(\omega, u, \phi)_{a}$.

Analogously as Section 4.2, we find a Fréchet differentiable functional of class $C^{1}$ whose derivatives are the Euler-Lagrange equations above, and that restricted to a certain domain its extremes are weak solutions for SBP- $a$. Then, we arrive to a problem of extremes on the constraint $M=\left\{(u, \phi) \in H_{0}^{1}(\Omega) \times \mathbb{H}_{\Delta^{2}}(\Omega) ; \quad\|u\|_{2}=1\right\}$ as we saw in Section 4.3.

Theorem 5.1. Let $a>0$. The triple $(\omega, u, \phi)_{a} \in \mathbb{R} \times M$ is a weak solution of the SBP-a system if, and only if, $(u, \phi)$ is a critical point of

$$
\left.F_{a}(u, \phi)\right|_{M}=\frac{1}{2} \int|\nabla u|^{2}+\frac{1}{2} \int \phi u^{2}-\frac{a^{2}}{4} \int|\Delta \phi|^{2}-\frac{1}{4} \int|\nabla \phi|^{2}
$$

having $\omega$ as a Lagrange multiplier.

Proof. It follows as well in the proof of Proposition 4.5.

Since $F_{a}$ is a functional that is unbounded, it is only possible to apply the theory of critical points after making a redefinition of $F_{a}$. Using the Riezs isomorphism $\left(a^{2} \Delta^{2}-\Delta\right)^{-1}$ we arrive to the appropriate functional given by

$$
J_{a}(u):=\frac{1}{2} \int_{\Omega}|\nabla u|^{2} d x+\frac{a^{2}}{4} \int_{\Omega}\left|\Delta \phi_{u}\right|^{2} d x+\frac{1}{4} \int_{\Omega}\left|\nabla \phi_{u}\right|^{2} d x,
$$

defined in $H_{0}^{1}(\Omega)$. As in Section 4.4, all the results in that section hold for this new functionals $F_{a}$ and $J_{a}$.

Recall that $B=\left\{u \in H_{0}^{1}(\Omega) ; \quad\|u\|_{2}=1\right\}$ is a level set on $H_{0}^{1}(\Omega)$. The restricted functional $\left.J_{a}\right|_{B}$ satisfies the necessary conditions to guarantee the existence of critical points, that is, the Palais-Smale condition and that of every sublevel set of $J_{a}$ has finite genus. This property of $\left.J_{a}\right|_{B}$ allow us to obtain the the necessary result to find the solutions of the system SBP- $a$.

Theorem 5.2. Let $\Omega$ be an open bounded set of $\mathbb{R}^{N}, N \geq 3$, and $a>0$. There exists a sequence $\left(\left(\omega_{n}, u_{n}, \phi_{n}\right)_{a}\right)_{n}$ such that $\left(\omega_{n}\right) \subset \mathbb{R}, \omega_{n} \rightarrow \infty$ and $\left(u_{n}, \phi_{n}\right) \in M$ solving the SBP-a system. Furthermore, $\left\|u_{n}\right\|_{H_{0}^{1}(\Omega)} \rightarrow \infty$.

\subsection{Regularity of solutions}

This section is devoted to show that every SBP-a system has classical solutions. Here, we deal with spaces of continuous solutions $C^{j, \lambda}$ introduced in Definition 2.9.

Theorem 5.3. Let $\Omega$ be an open bounded set of $\mathbb{R}^{N}, N \geq 3$, and $a>0$. If $(\omega, u, \phi)_{a} \in \mathbb{R} \times M$ is a weak solution of the $S B P-a$ system, then $u \in C^{2, \lambda}(\bar{\Omega})$ and $\phi \in C^{4, \lambda}(\bar{\Omega})$. 
Proof. We know that if $(u, \phi, \omega) \in M \times \mathbb{R}$ is a weak solution of SBP- $a$, then $\psi:=-a^{2} \Delta \phi+\phi$ is a weak solution on any bounded domain $\Omega$ of the Dirichlet problem

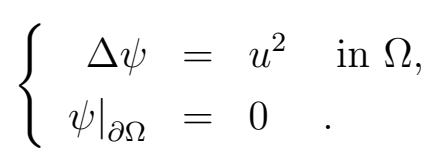

Now, if $u \in H_{0}^{1}(\Omega)$, then $u \in L^{6}(\Omega)$ by the Sobolev embedding we obtain

$$
\left(\int_{\Omega}|u|^{6}\right)^{\frac{2}{6}}=\left(\int_{\Omega}\left|u^{2}\right|^{3}\right)^{\frac{1}{3}}<\infty,
$$

i.e. $u^{2}$ belongs to $L^{3}(\Omega)$. Thus, by Theorem 2.26 we have

$$
-a^{2} \Delta \phi+\phi=\psi \in W^{2,3}(\Omega)
$$

Recall that $\Omega$ is a bounded set. If $\phi \in \mathbb{H}_{\Delta^{2}}(\Omega)$ is a solution of $(5.8)$ with $\psi \in W^{2,2}(\Omega)$, the interior regularity increases because Theorem 2.25 implies that $\phi \in W^{4,2}(\Omega)$ which leads us to the fact that $\phi \in C^{2, \lambda}(\bar{\Omega})$ with $\lambda \in\left(0, \frac{1}{2}\right]$ by the Sobolev embedding (2.8) of the Theorem 2.21 .

Now, considering the first equation of SBP-a

$$
-\Delta u+\phi u=\omega u \text { in } \Omega
$$

we have that $u \in H_{0}^{1}(\Omega)$ is the unique solution of $\Delta u=(\phi-\omega) u \in L^{2}(\Omega)$ because $\phi \in$ $C^{2, \lambda}(\bar{\Omega})$. Then, by Theorem 2.26

$$
\Delta u=(\phi-\omega) u \in H_{0}^{2}(\bar{\Omega})
$$

Therefore, Theorem 2.25 implies that $\phi \in H_{0}^{4}(\bar{\Omega})$ which leads us to the fact that $u \in C^{2, \lambda}(\bar{\Omega})$ with $\lambda \in\left(0, \frac{1}{2}\right]$ by PART II of Theorem 2.21.

Since $u \in H_{0}^{1}(\Omega)$ and $u \in C^{2, \lambda}(\bar{\Omega}), \lambda \in\left(0, \frac{1}{2}\right]$, we obtain

$$
-\Delta \psi=u^{2} \in H^{2}(\Omega)
$$

Furthermore, by Theorem 2.25 we get that $\psi \in H^{4}(\Omega)$ and we had defined that

$$
-a^{2} \Delta \phi+\phi=\psi
$$

then the interior regularity of $\phi$ increases by the same Theorem, i.e. $\phi \in H^{6}(\Omega)$. Finally, by PART II of the Sobolev embedding 2.21

$$
\phi \in H^{6}(\Omega) \hookrightarrow C^{4, \lambda}(\bar{\Omega})
$$


where $\lambda \in\left(0, \frac{1}{2}\right]$.

\subsection{The SBP- $a$ system and a classical Schrödinger-Poisson system}

The aim of this section is to show that the solution of the SBP- $a$ system converges to the solution of a classical Schrödinger-Poisson system as the parameter $a \rightarrow 0$.

Consider the classical Schrödinger-Poisson system given by

$$
\left\{\begin{aligned}
-\Delta u+\phi u & =\omega u \\
-\Delta \phi & =u^{2}
\end{aligned} \text { in } \Omega,\right.
$$

with the boundary conditions

$$
u=\phi=0 \quad \text { on } \quad \partial \Omega
$$

and normalizing condition

$$
\int_{\Omega} u^{2}=1
$$

where $\Omega$ is a bounded open subset of $\mathbb{R}^{N}, N \geq 3$. This system will be denominated SP system. Note that when $a=0$ the SBP-0 system becomes the system (5.9). V. Benci and D. Fortunato in [BF98] obtained the next result about system (5.9).

Theorem 5.4. Let $\Omega$ be a bounded and open set in $\mathbb{R}^{3}$. There is a sequence $\left(\left(u_{n}, \phi_{n}, \omega_{n}\right)_{0}\right)_{n} \in$ $H_{0}^{1}(\Omega) \times H_{0}^{1}(\Omega) \times \mathbb{R}$, with $\omega_{n}^{0} \rightarrow \infty$ and $\phi_{n}^{0}$ depending of $u_{n}^{0}$ i. e. $\phi_{n}^{0}=\phi_{u_{n}^{0}}^{0}$, solving the system (5.9).

On one hand, V. Benci and D. Fortunato in [BF98] show that the equation

$$
-\Delta \phi=u^{2}
$$

with the boundary and normalizing conditions above, has a unique weak solution. Namely, for every $u \in H_{0}^{1}(\Omega)$ is defined a continuous linear functional

$$
\begin{aligned}
g_{u}: H_{0}^{1}(\Omega) & \longrightarrow \mathbb{R} \\
v & \longmapsto g_{u}[v]=\int_{\Omega} u^{2} v d x .
\end{aligned}
$$

By Theorem 2.6 and Poincaré inequality there exists a unique vector $\phi_{u}^{0} \in H_{0}^{1}(\Omega)$ such that

$$
g_{u}[v]=\left(\phi_{u}^{0}, v\right)_{H_{0}^{1}(\Omega)} \quad \text { for all } v \in H_{0}^{1}(\Omega),
$$

where we denote by $\phi^{0}$ the function $\phi_{u}^{0} \in H_{0}^{1}(\Omega)$. 
On the other hand, in Proposition 4.6 we obtained that for every $u \in H_{0}^{1}(\Omega)$ there exists a unique vector $\phi^{a}:=\phi_{u}^{a} \in \mathbb{H}_{\Delta^{2}}(\Omega)$ such that

$$
f_{u}[v]=\left(\phi_{u}^{a}, v\right)_{\mathbb{H}_{\Delta^{2}}} \quad \text { for all } v \in \mathbb{H}_{\Delta^{2}}(\Omega)
$$

Thereby $\phi_{u}^{a}$ is the unique weak solution of the equation $\Delta^{2} \phi-\Delta \phi=u^{2}$ in $\Omega$ with the boundary and normalizing conditions given.

Now, we can present the next result of this section.

Lemma 5.1. Let $u^{0} \in H_{0}^{1}(\Omega)$ and $\left\{u^{a}\right\} \subset H_{0}^{1}(\Omega), a \in(0,1]$. Also, let

$$
\phi^{0} \in H_{0}^{1}(\Omega) \text { the unique weak solution of }-\Delta \phi=\left(u^{0}\right)^{2} \text { in } \Omega
$$

and

$$
\phi^{a} \in \mathbb{H}_{\Delta^{2}}(\Omega) \text { the unique weak solution of }-\Delta \phi+a^{2} \Delta^{2} \phi=\left(u^{a}\right)^{2} \text { in } \Omega \text {. }
$$

As $a \rightarrow 0$, we have:

1. if $u^{a} \rightarrow u^{0}$ in $H_{0}^{1}(\Omega)$, then $\phi^{a} \rightarrow \phi^{0}$ in $H_{0}^{1}(\Omega)$,

2. if $u^{a} \rightarrow u^{0}$ in $H_{0}^{1}(\Omega)$, then $\phi^{a} \rightarrow \phi^{0}$ in $H_{0}^{1}(\Omega)$ and $a \Delta\left(\phi^{a}\right) \rightarrow 0$ in $L^{2}(\Omega)$.

Proof. Let $\phi^{a} \in \mathbb{H}_{\Delta^{2}}(\Omega)$ be a weak solution of $-\Delta \phi+a^{2} \Delta^{2} \phi=\left(u^{a}\right)^{2}$ in $\Omega$, then it satisfies the Euler-Lagrange equation

$$
\int_{\Omega}\left|\nabla \phi^{a}\right|^{2}+a^{2} \int_{\Omega}\left|\Delta \phi^{a}\right|^{2}=\int_{\Omega}\left(u^{a}\right)^{2} \phi^{a}
$$

then

$$
\left\|\nabla \phi^{a}\right\|_{2}^{2}+a^{2}\left\|\Delta \phi^{a}\right\|_{2}^{2}=\left|\int_{\Omega}\left(u^{a}\right)^{2} \phi^{a}\right| .
$$

Arguing as in (4.43), we obtain

$$
\left\|\nabla \phi^{a}\right\|_{2}^{2}+a^{2}\left\|\Delta \phi^{a}\right\|_{2}^{2}=\left|\int_{\Omega}\left(u^{a}\right)^{2} \phi^{a}\right| \leq C_{1}\left\|u^{a}\right\|_{H_{0}^{1}}^{2}\left\|\phi^{a}\right\|_{2}
$$

Recall that $\phi^{a} \in \mathbb{H}_{\Delta^{2}}(\Omega)$. The Poincaré inequality (2.22) allows us to estimate $\left\|\phi^{a}\right\|_{2}$

$$
\left\|\nabla \phi^{a}\right\|_{2}^{2}+a^{2}\left\|\Delta \phi^{a}\right\|_{2}^{2} \leq C_{2}\left\|u^{a}\right\|_{H_{0}^{1}}^{2}\left\|\nabla \phi^{a}\right\|_{2}
$$

Since $\left\|\nabla \phi^{a}\right\|_{2}^{2} \leq C_{2}\left\|u^{a}\right\|_{H_{0}^{1}}^{2}\left\|\nabla \phi^{a}\right\|_{2}$, we obtain

$$
\left\|\nabla \phi^{a}\right\|_{2} \leq C_{2}\left\|u^{a}\right\|_{H_{0}^{1}}^{2}
$$


Also $a^{2}\left\|\Delta \phi^{a}\right\|_{2}^{2} \leq C_{2}\left\|u^{a}\right\|_{H_{0}^{1}}^{2}\left\|\nabla \phi^{a}\right\|_{2}$, this combined with the above inequality gives

$$
\left\|a \Delta \phi^{a}\right\|_{2} \leq C_{2}\left\|u^{a}\right\|_{H_{0}^{1}}^{2}
$$

Then, there exists $\phi_{*} \in H_{0}^{1}(\Omega)$ such that $\phi^{a} \rightarrow \phi_{*}$ in $H_{0}^{1}(\Omega)$, as $a \rightarrow 0$.

We will take the limit, when $a$ tends to zero, in the Euler-Lagrange equation

$$
\int_{\Omega} \nabla \phi^{a} \nabla \varphi+a^{2} \int_{\Omega} \Delta \phi^{a} \Delta \varphi=\int_{\Omega}\left(u^{a}\right)^{2} \varphi, \text { for all } \varphi \in \mathbb{H}_{\Delta^{2}}(\Omega) .
$$

By Hölder inequality we have

$$
0 \leq\left|a^{2} \int_{\Omega} \Delta \phi^{a} \Delta \varphi\right| \leq a\left\|\Delta \phi^{a}\right\|_{2}\|\Delta \varphi\|_{2} \leq a C
$$

then $\left|a^{2} \int_{\Omega} \Delta \phi^{a} \Delta \varphi\right| \rightarrow 0$ as $a \rightarrow 0$, and by Dominated Convergence Theorem 2.8 Equation (5.10) converges to

$$
\int_{\Omega} \nabla \phi_{*} \nabla \varphi=\int_{\Omega}\left(u^{0}\right)^{2} \varphi
$$

By the uniqueness of solution of $-\Delta \phi=\left(u^{0}\right)^{2}$ in $\Omega, \phi_{*}=\phi^{0}$. Then we have proved 1 .

Assume $u^{a} \rightarrow u^{0}$ in $H_{0}^{1}(\Omega)$, then $u^{a} \rightarrow u^{0}$ in $H_{0}^{1}(\Omega)$, thus Claim 1 implies that $\phi^{a} \rightarrow \phi^{0}$ in $H_{0}^{1}(\Omega)$. As a consequence we have

$$
\left\|\nabla \phi^{0}\right\|_{2}^{2} \leq \lim \inf _{a \rightarrow 0}\left\|\nabla \phi^{a}\right\|_{2}^{2}
$$

Let $\left\{\varphi_{n}\right\} \in C_{0}^{\infty}(\Omega)$ be a sequence such that $\varphi_{n} \rightarrow \phi^{0}$ in $H_{0}^{1}(\Omega)$ as $n \rightarrow+\infty$. By Lax-Milgram Theorem $2.7 \phi^{a}$ minimizes the functional

$$
E_{a}(\phi)=\frac{1}{2}\|\nabla \phi\|_{2}^{2}+\frac{a^{2}}{2}\|\Delta \phi\|_{2}^{2}-f_{u^{a}}[\phi], \text { with } \phi \in \mathbb{H}_{\Delta^{2}}(\Omega) .
$$

Since $E_{a}\left(\phi^{a}\right)$ is a minimum value, then

$$
\begin{aligned}
\frac{1}{2}\left\|\nabla \phi^{a}\right\|_{2}^{2} & =E_{a}\left(\phi^{a}\right)-\frac{a^{2}}{2}\left\|\Delta \phi^{a}\right\|_{2}^{2}+f_{u^{a}}\left[\phi^{a}\right] \\
& \leq E_{a}\left(\varphi_{n}\right)+f_{u^{a}}\left[\phi^{a}\right] \\
& =\frac{1}{2}\left\|\nabla \varphi_{n}\right\|^{2}+\frac{a^{2}}{2}\left\|\Delta \varphi_{n}\right\|_{2}^{2}-f_{u^{a}}\left[\varphi_{n}\right]+f_{u^{a}}\left[\phi^{a}\right]
\end{aligned}
$$

and thereby

$$
\limsup _{a \rightarrow 0} \frac{1}{2}\left\|\nabla \phi^{a}\right\|_{2}^{2} \leq \frac{1}{2}\left\|\nabla \varphi_{n}\right\|_{2}^{2}-g_{u^{0}}\left[\varphi_{n}\right]+g_{u^{0}}\left[\phi^{0}\right]
$$


Taking the limit, when $n$ is going to zero, we have

$$
\limsup _{a \rightarrow 0}\left\|\nabla \phi^{a}\right\|_{2}^{2} \leq\left\|\nabla \phi^{0}\right\|_{2}^{2}
$$

From the inequalities (5.11) and (5.12), and the convergence $\phi^{a} \rightarrow \phi^{0}$ in $H_{0}^{1}(\Omega)$, we infer $\phi^{a} \rightarrow \phi^{0}$ in $H_{0}^{1}(\Omega)$. Finally, we can see that when $a \rightarrow 0$

$$
\left\|a \Delta \phi^{a}\right\|_{2}^{2}=f_{u^{a}}\left[\phi^{a}\right]-\left\|\nabla \phi^{a}\right\|_{2}^{2} \rightarrow g_{u^{0}}\left[\phi^{0}\right]-\left\|\nabla \phi^{0}\right\|_{2}^{2}=0
$$

and the proof is complete.

Theorem 5.2 concluded that there exists a sequence $\left(\left(\omega_{n}, u_{n}, \phi_{n}\right)_{a}\right)_{n} \in \mathbb{R} \times H_{0}^{1}(\Omega) \times$ $\mathbb{H}_{\Delta^{2}}(\Omega)$ of solutions to the SBP- $a$ system. In the next result, we will show that for each $n$

$$
\lim _{a \rightarrow 0}\left(\omega_{n}^{a}, u_{n}^{a}, \phi_{n}^{a}\right)=\left(\omega_{n}^{0}, u_{n}^{0}, \phi_{n}^{0}\right),
$$

where $\left(\omega_{n}^{0}, u_{n}^{0}, \phi_{n}^{0}\right)$ solves the SP system in (5.9).

Theorem 5.5. Let $\left(\omega^{a}, u^{a}, \phi^{a}\right) \in \mathbb{R} \times H_{0}^{1}(\Omega) \times \mathbb{H}_{\Delta^{2}}(\Omega)$ be a solution of the SBP-a system 5.1. Then $\omega^{a} \rightarrow \omega^{0}$ in $\mathbb{R}, u^{a} \rightarrow u^{0}$ in $H_{0}^{1}(\Omega)$ and $\phi^{a} \rightarrow \phi^{0}$ in $H_{0}^{1}(\Omega)$ as $a \rightarrow 0$ where $\left(\omega^{0}, u^{0}, \phi^{0}\right) \in \mathbb{R} \times H_{0}^{1}(\Omega) \times H_{0}^{1}(\Omega)$ of the SP system.

Proof. Fix $n \in \mathbb{N}$. Let $\left\{\left(\omega^{a}, u^{a}, \phi^{a}\right)\right\}_{a \in(0,1)} \subset \mathbb{R} \times H_{0}^{1}(\Omega) \times \mathbb{H}_{\Delta^{2}}(\Omega)$ be a family of the solutions of SBP-a in 5.1. Then, the every function of the set $\left\{u^{a}\right\}_{a}$ solve

$$
-\Delta u+\phi^{a} u=\omega^{a} u \quad \text { in } \Omega
$$

respectively. Also, every function $u^{a}$ is respectively the critical point of $J_{a}$ in (5.7), characterized by

$$
b_{n}^{a}:=\inf \left\{\sup J_{a}(A) ; A \in \mathcal{A}_{k+1}\right\} \geq n>0,
$$

where $\sup J_{a}(A):=\left\{\sup J_{a}(u): u \in A\right\}, k$ is an integer that depends on $n$ and $\mathcal{A}_{k+1}$ is a appropriated collection of sets defined by

$$
\mathcal{A}_{k+1}:=\{A \subset B ; \quad A \text { is symmetric and closed with } \gamma(A)=k+1\} .
$$

We can see more details about this characterization on Section 4.6. A critical point $b_{n}^{0}$ of $J_{0}$ has a similar characterization in V. Benci and D. Fortunato in [BF98], where

$$
J_{0}(u):=\frac{1}{2} \int_{\Omega}|\nabla u|^{2} d x+\frac{1}{4} \int_{\Omega}\left|\nabla \phi_{u}\right|^{2} d x .
$$

is the reduced functional related to SP. 
Recall that if $u^{a}$ is a solution of $-\Delta u+\phi^{a} u=\omega^{a} u$ in $\Omega$, provided that $\phi^{a}=\Phi\left(u^{a}\right)$ and $\phi^{a}$ belongs to $\mathbb{H}_{\Delta^{2}}(\Omega) \subset H_{0}^{1}(\Omega)$, then the space where stay the solutions is contained in the space that contents solutions of $-\Delta u+\phi^{0} u=\omega^{0} u$ in $\Omega$. However, if a solution of the last equation $u^{0}$ such that $\phi^{0}$ belongs to $H_{0}^{1}(\Omega)$ but not to $H_{2}(\Omega)$, then $u^{0}$ is not a solution for a SBP-a system. For that reason, we obtain

$$
\inf \left\{\sup J_{0}(A), A \in \mathcal{A}_{k+1}\right\}=b_{0} \geq \inf \left\{\sup J_{a}(A), A \in \mathcal{A}_{k+1}\right\} \geq n>0,
$$

then, there exists $u^{a}$ such that

$$
J_{a}^{\prime}\left(u^{a}\right)\left[u^{a}\right]=\int_{\Omega}\left|\nabla u^{a}\right|^{2} d x+a^{2} \int_{\Omega}\left|\Delta \phi^{a}\right|^{2} d x+\int_{\Omega}\left|\nabla \phi^{a}\right|^{2} d x=\omega^{a}
$$

and we can estimate

$$
\begin{aligned}
b_{n}^{0} \geq b_{n}^{a} & =J_{a}\left(u^{a}\right) \\
& =\frac{1}{2} \omega^{a}-\frac{a^{2}}{4} \int_{\Omega}\left|\Delta \phi^{a}\right|^{2} d x-\frac{1}{4} \int_{\Omega}\left|\nabla \phi^{a}\right|^{2} d x \\
& =\frac{1}{2} \int_{\Omega}\left|\nabla u^{a}\right|^{2} d x+\frac{a^{2}}{4} \int_{\Omega}\left|\Delta \phi^{a}\right|^{2} d x+\frac{1}{4} \int_{\Omega}\left|\nabla \phi^{a}\right|^{2} d x \\
& \geq \frac{1}{2}\left\|\nabla u^{a}\right\|_{2}^{2} .
\end{aligned}
$$

With the inequality above an the Poincaré inequality (2.22) we obtain that $\left\{u^{a}\right\}$ is bounded in the $H_{0}^{1}(\Omega)$ norm

$$
\frac{2 b_{n}^{0}}{C} \geq\left\|\nabla u^{a}\right\|_{2}^{2} \geq\left\|u^{a}\right\|_{H_{0}^{1}(\Omega)}^{2}
$$

where $b_{0}$ does not depend on $a$. Therefore, there exists $u^{0} \in H_{0}^{1}(\Omega)$ such that, up to subsequences, $u^{a} \rightarrow u^{0}$ in $H_{0}^{1}(\Omega)$ as $a \rightarrow 0$.

Also, observe that

$$
b_{n}^{0} \geq \frac{1}{2} \int_{\Omega}\left|\nabla u^{a}\right|^{2} d x+\frac{a^{2}}{4} \int_{\Omega}\left|\Delta \phi^{a}\right|^{2} d x+\frac{1}{4} \int_{\Omega}\left|\nabla \phi^{a}\right|^{2} d x \geq \frac{1}{4} \omega^{a},
$$

then $\left\{\omega^{a}\right\}_{a}$ is bounded. Then, there exists a $\omega^{0} \in \mathbb{R}$ and up to subfamily

$$
\lim _{a \rightarrow 0} \omega^{a}=\omega^{0}
$$

Now, we consider the equation

$$
-\Delta u^{a}+\phi^{a} u^{a}=\omega^{a} u^{a} \quad \text { in } \quad \Omega,
$$


applying the inverse Riesz isomorphism $\Delta^{-1}: H^{-1}(\Omega) \rightarrow H_{0}^{1}(\Omega)$, we obtain

$$
-u^{a}+\Delta^{-1} \phi^{a} u^{a}=\omega^{a} \Delta^{-1} u^{a}
$$

and since $u^{a} \rightarrow u^{0}$ in $H_{0}^{1}(\Omega)$ as $a \rightarrow 0$ and by part 1 . of Lemma $5.1 \phi^{a} \rightarrow \phi^{0}$, then $u^{a} \rightarrow u^{0}$ in $H_{0}^{1}(\Omega)$ as $a \rightarrow 0$.

Thus, we can use the part 2. of Lemma 5.1 and obtain that $\phi^{a} \rightarrow \phi^{0}$, where $\phi^{0} \in H_{0}^{1}(\Omega)$ is the unique solution of $-\Delta \phi=\left(u^{0}\right)^{2}$.

Finally, let $\varphi \in C_{0}^{\infty}(\Omega)$. Then

$$
\int_{\Omega} \nabla u^{a} \nabla \varphi+\int_{\Omega} \phi^{a} u^{a} \varphi=\omega^{a} \int_{\Omega}\left(u^{a}\right)^{2}
$$

Take limit as $a \rightarrow 0$ in each term implies the convergence

$$
\int_{\Omega} \nabla u^{a} \nabla \varphi+\int_{\Omega} \phi^{a} u^{a} \varphi=\omega^{a} \int_{\Omega}\left(u^{a}\right)^{2} \rightarrow \int_{\Omega} \nabla u^{0} \nabla \varphi+\int_{\Omega} \phi^{0} u^{0} \varphi=\omega^{0} \int_{\Omega}\left(u^{0}\right)^{2} .
$$

The convergence of each term follows as in Lemma 5.1. 


\section{Glossary}

$(P S)$ Palais-Smale condition. 22

$(P S)_{c}$ Palais-Smale condition at level c. 22

$C_{0}^{\infty}(\Omega)$ Continuously differentiate functions with compact suppo. 10

$C^{j, \lambda}(\bar{\Omega})$ functions in $C^{j}(\Omega)$ such that for every multi-index $\alpha$ with $|\alpha| \leq j$ th function $x \rightarrow D^{\alpha} u(x)$ admits a continuous extension to $\bar{\Omega}$, and $D^{\alpha} u$ satisfies in $\Omega$ a Hölder condition with exponent $\lambda .10$

$C_{B}^{j}(\Omega)$ functions in $C^{j}(\Omega)$ such that for every multi-index $\alpha$ with $|\alpha| \leq j D^{\alpha} u$ is bounded on $\Omega .10$

$H^{1}(\Omega)$ Sobolev Space. 11

$H_{0}^{1}(\Omega)$ Sobolev Space. 12

$H^{2}(\Omega)$ Sobolev Space. 12

$L^{p}(\Omega)$ measurable functions space. 8

$W^{m, p}(\Omega)$ Sobolev Space. 11

$\mathbb{H}_{\Delta^{2}}(\Omega)$ intesrection of $H_{0}^{1}(\Omega) \cap H^{2}(\Omega) .42$

$\Omega$ open and bounded set of $\mathbb{R}^{N}$.

$\gamma(A)$ genus of a closed and symmetric Banach subset A. 18

$\partial \Omega$ boundary of $\Omega$.

SBP System of Schrödinger-Bopp-Podolsky equations. 39

SBP- $a$ System of Schrödinger-Bopp-Podolsky equations with parameter $a .57$

SP System of Schrödinger-Poisson equations. 61 


\section{Bibliography}

[AF03] R. A. Adams and John Fournier. Sobolev Spaces. Academic Press, second edition, 2003. 10,11

[AR73] A. Ambrosetti and P. H. Rabinowitz. Dual variational methods in critical point theory and applications. Journal of Functional Analysis, (14):349-381, 1973. 26

[BB92] P. Blanchard and E. Brüning. Variational Methods in Mathematical Phisics. A Unified Approach. Texts and Monographs in Physics, 1992. 19, 21

[BF98] V. Benci and D. Fortunato. An eigenvalue problem for the Schrödinger-Maxwell equations. Topological Methods in Nonlinear Analysis, 11:283-293, 1998. 3, 46, 61,64

[Bop40] F. Bopp. Eine lineare theorie des elektrons. Ann. Phys., 1940. 34

[Bre11] H. Brezis. Functional Analysis, Sobolev Spaces and Partial Differential Equations. Universitext, 2011. 7, 11

[Cla72] D.C. Clarck. A variant of the Ljusternick-Schnirelman Theory and nonlinear eigenvalue problems. Mathematical Annals, (199):55-72, 1972. 24

[COAM01] João Marcos do Ò C. O. Alves and O. H. Miyagaki. Nontrivial solutions for a class of semilinear biharmonic problems involving critical exponents. Nonliinear Analisis, (46):121-133, 2001. 1

[Cof69] C.V. Coffman. A minimum-maximum principle for a class of nonlinear integral equations. Jornal Analyse Math., (22):391-419, 1969. 18

[Die69] J. Dieudonné. Foundations of Modern Analysis, volume 1. Academic Press, 1969. 5

[dS19] P. d'Avenia and G. Siciliano. Nonlinear Schrödinger equation in the BoppPodolsky electrodinamics: Solutions in the electrostatic case. Journal of the Differential Equations, (267):1025-1065, 2019. 1, 35, 57

[Eke74] I. Ekeland. On the variational principle. Journal of Mathematical Analysis and Application, (47):324-353, 1974. 29

[GT88] D. Gilbarg and N. S. Trudinger. Elliptic Partial Differential Equations of Second Order. Classics in Matematics, 1988. 10, 17

[Heb20] E. Hebey. Strong convergence of the Bopp-Podolsky-Schrödinger-Proca system to the Schrödinger-Poisson-Proca system in the electro-magneto-static case. Calculus of Variations, 2020. 2 
[Kav93] O. Kavian. Introduction à la Thèorie des Points Critiques et Applications aux Problèmes Elliptiques. Verlag, 1993. 21

[Kes89] S. Kesavan. Topics in Functional Analysis and Applications. 1989. 11, 26

[Kre78] E. Kreyszig. Introductory Functional Analysis with Applications. 1978. 7

[LS34] L. Ljusternik and L. Schnirelman. Métodes topologiques dans les problèmes variationnels. Hermann, Paris, 1934. 19, 20, 23

[Pod42] B. Podolsky. A generalized electrodynamics. Phys. Rev., 1942. 34, 36

[Rab74] P. H Rabinowitz. Variational methods for nonlinear eigenvalue problems. In Proc. CIME, 1974. 18

[Shw69] Jacob T. Shwartz. Nonlinear Functional Analysis. 1969. 5, 19

[Str90] M. Struwe. Variational Methods and Their Applications to Non-linear Partial Differential Equations and Hamiltonian Systems. Verlag, 1990. 18

[Zei84] E Zeidler. Nonlinear Functional Analysis and its applications, volume III: Variational Methods and Optimization. Verlag, 1984. 19, 21

[Zei88] E Zeidler. Nonlinear Functional Analysis and its Applications, volume IV: Applications to Mathematical Physic. Verlag, 1988. 19 